Systematic Review

\title{
e A Systematic Evaluation of Prevalence and Diagnostic Accuracy of Sacroiliac Joint Interventions
}

Thomas T. Simopoulos, MD¹, Laxmaiah Manchikanti, MD², Vijay Singh, MD², Sanjeeva Gupta, MD ${ }^{4}$, Haroon Hameed, MD5 , Sudhir Diwan, MD' ${ }^{6}$, and Steven P. Cohen, $\mathrm{MD}^{7}$

From: ${ }^{1}$ Beth Israel Deaconess Medical Center, and Harvard Medical School, Boston, MA;

${ }^{2}$ Pain Management Center of

Paducah, Paducah, KY, and University of Louisville,Louisville, KY; ${ }^{3}$ Pain Diagnostics Associates, Niagara, WI; ${ }^{4}$ Bradford Teaching Hospitals NHS Foundation Trust,

Bradford, United Kingdom; 5Johns Hopkins University School

of Medicine, Baltimore, MD;

${ }^{6}$ The Spine And Pain Institute of New York, New York, New York; and 7Johns Hopkins School of Medicine, Baltimore, MD, and Uniformed Services University of the Health Sciences, Bethseda, MD

See Additional Author Afffiliations on page $E_{335}$

Address correspondence: Thomas T. Simopoulos, MD Beth Israel Deaconess One Brookline Place, Ste. 105 Boston, MA 02445

E-mail: tsimopou@bidmc. harvard.edu

Disclaimer: There was no external funding in the preparation of this manuscript.

Conflict of interest: None.

Manuscript received: $02 / 12 / 2012$ Revised manuscript received: 03/12/2012

Accepted for publication: 04/09/2012

Free full manuscript: www.painphysicianjournal.com
Background: The contributions of the sacroiliac joint to low back and lower extremity pain have been a subject of considerable debate and research. It is generally accepted that $10 \%$ to $25 \%$ of patients with persistent mechanical low back pain below $L 5$ have pain secondary to sacroiliac joint pathology. However, no single historical, physical exam, or radiological feature can definitively establish a diagnosis of sacroiliac joint pain. Based on present knowledge, a proper diagnosis can only be made using controlled diagnostic blocks. The diagnosis and treatment of sacroiliac joint pain continue to be characterized by wide variability and a paucity of the literature.

Objective: To evaluate the accuracy of diagnostic sacroiliac joint interventions

Study Design: A systematic review of diagnostic sacroiliac joint interventions.

Methods: Methodological quality assessment of included studies was performed using Quality Appraisal of Reliability Studies (QAREL). Only diagnostic accuracy studies meeting at least $50 \%$ of the designated inclusion criteria were utilized for analysis. Studies scoring less than $50 \%$ are presented descriptively and analyzed critically.

The level of evidence was classified as good, fair, or limited (or poor) based on the quality of evidence developed by the United States Preventive Services Task Force (USPSTF).

Data sources included relevant literature identified through searches of PubMed and EMBASE from 1966 to December 2011, and manual searches of the bibliographies of known primary and review articles.

Outcome Measures: In this evaluation we utilized controlled local anesthetic blocks using at least $50 \%$ pain relief as the reference standard.

Results: The evidence is good for the diagnosis of sacroiliac joint pain utilizing controlled comparative local anesthetic blocks. The prevalence of sacroiliac joint pain is estimated to range between $10 \%$ and $62 \%$ based on the setting; however, the majority of analyzed studies suggest a point prevalence of around $25 \%$, with a false-positive rate for uncontrolled blocks of approximately $20 \%$. The evidence for provocative testing to diagnose sacroiliac joint pain was fair. The evidence for the diagnostic accuracy of imaging is limited.

Limitations: The limitations of this systematic review include a paucity of literature, variations in technique, and variable criterion standards for the diagnosis of sacroiliac joint pain.

Conclusions: Based on this systematic review, the evidence for the diagnostic accuracy of sacroiliac joint injections is good, the evidence for provocation maneuvers is fair, and evidence for imaging is limited.

Key words: Chronic low back pain, sacroiliac joint pain, sacroiliitis, sacroiliac joint injection, sacroiliac joint dysfunction, provocation manuevers, controlled diagnostic blocks, intraarticular injection, extraarticular injection.

Pain Physician 2012; 15:E305-E344 


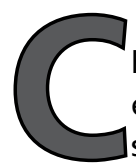
hronic low back pain, with or without lower extremity pain, that arises from various structures of the spine constitutes a majority of pain complaints (1-11). The high prevalence of chronic low back pain, the numerous modalities of treatments for managing the problem, and the growing social and economic costs continue to influence medical decision-making $(1,2,5,12-35)$. Even though low back pain is a common complaint in primary care and tertiary care, it is often difficult to reach a definitive diagnosis (2,35-45). Controlled studies have established intervertebral discs, facet joints, and sacroiliac joints as potential sources of low back and lower extremity pain (2,35-49). Thus, the sacroiliac joint is accepted as a potential source of low back and/or buttock pain with or without lower extremity pain $(2,36,39,41-44,46$ 53). The sacroiliac joint has been implicated as the primary source of pain $(2,36,39,44,47-49,53)$ in $10 \%$ to $27 \%(41,54,55)$ of patients with mechanical low back pain below L5 utilizing controlled, comparative local anesthetic blocks.

A major source of the exponential growth in treatment modalities is the inherent difficulty in obtaining an accurate diagnosis (1-5,14-36,56-71). An inaccurate or incorrect diagnosis may lead not only to treatment failure, but also results in wasted health care dollars, diverting essential health care resources. Fundamental to an accurate diagnosis is the reliability of the test used to make the diagnosis $(2,39,40,44,47,48,49,53,72-$ 77). Attempts have been made to improve the accuracy of diagnosing sacroiliac joint pain by multiple means, including physical examination, imaging techniques, and controlled local anesthetic blocks $(2,36,39,41,42,44,47,49,53-55,78-84)$.

However, there is no universally accepted gold standard for the diagnosis of low back pain, regardless of whether the suspected source is the sacroiliac joint(s), intervertebral disc(s), or facet joint(s) $(2,35$ 49,53-55,79-86). The recommended reference standards typically involve anesthetic or provocative injections $(2,35-49,53)$. Multiple arguments have been made in favor of and against the diagnostic accuracy of controlled local anesthetic blocks $(2,27,39-49,53,74,75,85-$ $89)$, but controlled local anesthetic blocks continue to be the best available tool to identify intervertebral discs, facet, or sacroiliac joint(s) as the source of low back pain. Yet, these reference standards are invasive, expensive, and often difficult to interpret, and therefore may not be suitable for routine clinical use as a primary diagnostic modality.
The sacroiliac joint is a true diarthrodial joint; matching articular surfaces separated by a joint space containing synovial fluid and enveloped by a fibrous capsule, but, with unique characteristics not typically found in other diarthrodial joints (90-96). The sacroiliac joint contains fibrocartilage in addition to hyaline cartilage (97), and is characterized by discontinuity of the posterior capsule, with ridges and depressions that minimize movement and enhance stability (51). Consequently, the sacroiliac joint has been described as a true synovial joint only in the anterior portion. In contrast, the posterior connection is a syndesmosis consisting of the ligamenta sacroiliaca, the musculus gluteus medius and minimus, and the musculus pyriformis (57).

The sacroiliac joint is well imbued with nociceptor and proprioceptors. Information on the innervation pattern is the subject of considerable controversy. Solonen (98) examined data from earlier studies (1857-1944) that collectively identified branches from the lumbosacral plexus, superior gluteal nerve, dorsal rami of S1 and S2, and the obturator nerve, as providing innervation. But despite multiple studies (99-105), the exact innervation continues to be unclear. The anterior portion may be innervated by the sacral plexus, whereas the posterior portion may have innervation from the spinal nerves. It has been proposed that the predominant innervation is via the $L 4$ to $\$ 1$ nerve roots, with some contribution from the superior gluteal nerve (106). Even though there may be input from ventral rami, several authors have argued that the joint is innervated only by the sacral dorsal rami $(104,107)$. Bernard (108) proposed that the posterior innervation is from the lateral branches of the posterior rami of L4 to S3, whereas the anterior innervation stems from the L2 to S2 segments.

Nakagawa (109) reported that the nerve filaments to the joint are derived from the ventral rami of $L 4$ and $L 5$, the superior gluteal nerve, and the dorsal rami of the L5, S1, and S2. In contrast, Grob et al (104) found that the innervation of the sacroiliac joint is almost exclusively derived from the sacral dorsal rami. Dissections of fetal pelvises confirmed that innervation of the sacroiliac joint originates in the dorsal rami because neural filaments are noted solely in the dorsal mesenchyme $(107,109)$.

Murata et al (101) evaluated the sensory innervation of the sacroiliac joint in rats and concluded that the sacroiliac joint was innervated by sensory neurons in dorsal root ganglia ipsilateral to the joint from the L1 to S2. They also concluded that sensory fibers from 
the L1 and L2 dorsal root ganglia passed through the paravertebral sympathetic trunk.

Histologic analyses of chronically painful sacroiliac joints has verified the presence of nerve fibers within the joint capsule and adjoining ligaments $(107,110,111)$. A recent cadaveric study by McGrath and Zhang (112) found that the long posterior sacroiliac ligament received afferent input from S2 (96\%) and S3 (100\%) in almost all specimens, from $\mathrm{S} 4$ in $59 \%$ of cases, and only occasionally from S1 (4\%). The nerve fascicles contain both myelinated and unmyelinated nerve fibers, 2 morphotypes of paciniform-encapsulated mechanoreceptors, and a single nonpaciniform mechanoreceptor, suggesting that both pain and proprioception are transmitted from the sacroiliac joint $(99,103,110,111,113)$. Szadek et al (105) concluded that the presence of calcitonin gene-related peptide and substance $P$ immunoreactive fibers in the anterior capsular and interosseous ligaments provide a morphological and physiological base for pain signals originating from these structures. They further hypothesized that infiltration techniques used to diagnose sacroiliac joint pain should consider extraarticular as well as intraarticular approaches. Sakamoto et al (103) showed that most mechanoreceptor units in the sacroiliac joint are high-threshold group 3 units that likely serve a nociceptive function. However, they contend that the sacroiliac joint has little proprioceptive function.

Forst et al (51) described extensive communication between the sacroiliac joint and nearby neural structures. Patterns of extracapsular extravasation from the sacroiliac joint have been observed on postarthrography computed tomography (CT) (114). These patterns include posterior extension into the dorsal sacral foramina, extravasation into the L5 epiradicular sheath via the superior recess, and ventral leakage into the lumbosacral plexus $(111,114)$. Thus, it is plausible that in the setting of capsular disruption, inflammatory mediators could leak out from the sacroiliac joint into nearby neural structures, causing radicular pain in certain patients $(111,114)$.

Several mechanisms of injury have been linked to the development of sacroiliac joint pain, including a direct fall on the buttocks, a rear-end or broad-side type motor vehicle accident, and an unanticipated step into a hole or from a miscalculated height $(90,91)$. In a study performed in 54 patients with suspected sacroiliac joint syndrome, Chou et al (115) found that $44 \%$ of patients cited a specific traumatic event, $21 \%$ reported a cumulative injury, and $35 \%$ had had spontaneous or idiopathic onset of sacroiliac joint pain. Among the various inciting events, motor vehicle accidents and falls comprise a majority $(82,115,116)$. Other described causes include fusion surgery $(83,117-119)$, anterior dislocation (120), inflammatory and degenerative sacroiliac joint disease (121), and multiple other etiologies $(36,46,47,50,78,79,122,123)$. In a study by $\mathrm{Ha}$ et al $(124)$, the authors found that sacroiliac joint degeneration is nearly universal 5 years following fusion to the sacrum, and considerably more common than in non-operated controls after floating fusions.

In a systematic review evaluating a battery of tests to identify the disc, sacroiliac joint, or facet joint as the source of low back pain, Hancock et al (49) suggested that a combination of sacroiliac joint pain provocative maneuvers appears to be useful in pinpointing the sacroiliac joint as the principal source of symptoms in patients with pain below the 5 th lumbar vertebra. A systematic review by Szadek et al (80) showed that the thigh thrust test, the compression test, and 3 or more positive stressing tests contain sufficient discriminative power for diagnosing sacroiliac joint pain. A systematic literature review performed by Song et al (81) concluded that scintigraphy is of limited value at best in establishing sacroiliitis in patients with ankylosing spondylitis. In a best-evidence review of diagnostic procedures for neck and low back pain, Rubinstein and van Tulder (44) concluded that there was moderate evidence for the validity and accuracy of injections identified 3 systematic reviews $(49,125,126)$. An evidence-based review by Laslett (53) determined that among chronic back pain patients, the presence of 3 or more positive provocation sacroiliac joint tests in conjunction with the absence of "centralization" are associated with a $77 \%$ probability of sacroiliac joint pain, $89 \%$ in pregnant women. In contrast, in an evidence-based medicine series, Vanelderen et al (57) concluded that it was difficult to distinguish sacroiliac joint pain from other forms of low back pain based on history and physical exam alone. They also reported that provocative maneuvers have weak predictive value, though combined batteries of tests can help ascertain a diagnosis.

The primary purpose of this review is to systematically assess the literature on diagnostic sacroiliac joint interventions. The secondary objectives are to analyze studies for quality, and factors that can affect generalizability.

\subsection{Methods}

The methodology utilized in this systematic review 
followed the review process derived from evidencebased systematic reviews and meta-analysis of diagnostic accuracy studies $(22,44,49,72-77,127,128)$.

\subsection{Criteria for Considering Studies for This Review}

\subsubsection{Types of Studies}

Diagnostic accuracy studies evaluating sacroiliac joint pain

\subsubsection{Types of Participants}

Participants of interest were adults aged at least 18 years with chronic low back and lower extremity pain of at least 3 months duration.

Participants must have failed previous pharmacotherapy, exercise therapy, etc., prior to starting diagnostic interventional pain management techniques.

\subsubsection{Types of Interventions}

The interventions were diagnostic sacroiliac joint interventions appropriately performed with proper technique under fluoroscopic or CT guidance.

\subsubsection{Types of Outcome Measures}

- The primary outcome parameter was pain relief concordant with the type of controlled diagnostic blocks performed.

- The secondary outcome measures were the ability to perform previously painful movements without significant pain or complications.

- At least 2 of the review authors independently, in an unblinded standardized manner, assessed the outcomes measures. Any disagreements between reviewers were resolved by a third author and consensus.

\subsection{Literature Search}

Searches were performed from the following sources without language restrictions:

1. PubMed from 1966 www.ncbi.nlm.nih.gov/sites/entrez?db=pubmed

2. EMBASE from 1980 www.embase.com/

3. Cochrane Library www.thecochranelibrary.com/view/0/index.html

4. U.S. National Guideline Clearinghouse (NGC) www.guideline.gov/

5. Previous systematic reviews and cross references

6. Clinical Trials clinicaltrials.gov/

The search period was from 1966 through December 2011.

\subsection{Search Strategy}

The search strategy emphasized chronic low back pain, sacroiliac joint pain/arthritis, and diagnostic sacroiliac joint interventions and techniques.

This systematic review focused only on diagnostic studies, including invasive and noninvasive techniques and reports of complications. Only sacroiliac joint injections performed under fluoroscopy or CT imaging techniques were evaluated. Interventional techniques performed blindly or using other identification modalities were excluded. All studies describing appropriate outcome evaluations with proper statistical evaluations were reviewed. Reports without appropriate diagnosis, nonsystematic reviews, book chapters, and case reports were excluded.

At least 2 of the review authors independently, in an unblinded standardized manner, performed each search. Accuracy was confirmed by a statistician. All searches were combined to obtain a unified search strategy. Any disagreements between reviewers were resolved by a third author and consensus.

\subsection{Data Collection and Analysis}

The quality of each individual article used in this assessment was based on Quality Appraisal of Reliability Studies (QAREL) checklist (Table 1) (73). This checklist has been validated and utilized in multiple systematic reviews (73). Each study in the final sample of eligible manuscripts was assessed using a 12-item appraisal checklist designed to assess the quality and applicability of studies. The face validity of these checklists was established by consultation with methodology experts (73) and comparison with quality appraisal checklists used in other systematic reviews examining diagnostic reliability (129-134). This checklist was also developed in accordance to the Standards for Reporting Studies of Diagnostic Accuracy (STARD) (76), and the Quality Assessment of Diagnostic Accuracy Studies (QUADAS) $(76,77)$ appraisal tool. Studies were not given an overall numeric quality score; instead, each item was considered separately and graded as "yes," "no," "unclear," or "not applicable."

\subsubsection{Selection of Studies}

- In an unblinded standardized manner, 2 review authors screened the abstracts of all identified studies 
Table 1. Quality Appraisal of Diagnostic Reliability (QAREL) checklist.

\begin{tabular}{|c|c|c|c|c|}
\hline Item & Yes & No & Unclear & N/A \\
\hline \multicolumn{5}{|l|}{$\begin{array}{l}\text { 1. Was the test evaluated in a spectrum of subjects representative of patients who } \\
\text { would normally receive the test in clinical practice? }\end{array}$} \\
\hline \multicolumn{5}{|l|}{$\begin{array}{l}\text { 2. Was the test performed by examiners representative of those who would normally } \\
\text { perform the test in practice? }\end{array}$} \\
\hline \multicolumn{5}{|l|}{ 3. Were raters blinded to the reference standard for the target disorder being evaluated? } \\
\hline \multicolumn{5}{|l|}{ 4. Were raters blinded to the findings of other raters during the study? } \\
\hline \multicolumn{5}{|l|}{ 5. Were raters blinded to their own prior outcomes of the test under evaluation? } \\
\hline \multicolumn{5}{|l|}{$\begin{array}{l}\text { 6. Were raters blinded to clinical information that may have influenced the test } \\
\text { outcome? }\end{array}$} \\
\hline \multicolumn{5}{|l|}{$\begin{array}{l}\text { 7. Were raters blinded to additional cues, not intended to form part of the diagnostic } \\
\text { test procedure? }\end{array}$} \\
\hline \multicolumn{5}{|l|}{ 8. Was the order in which raters examined subjects varied? } \\
\hline \multicolumn{5}{|l|}{ 9. Were appropriate statistical measures of agreement used? } \\
\hline \multicolumn{5}{|l|}{ 10. Was the application and interpretation of the test appropriate? } \\
\hline \multicolumn{5}{|l|}{$\begin{array}{l}\text { 11. Was the time interval between measurements suitable in relation to the stability of } \\
\text { the variable being measured? }\end{array}$} \\
\hline \multicolumn{5}{|l|}{ 12. If there were dropouts from the study, was this less than $20 \%$ of the sample? } \\
\hline TOTAL & & & & \\
\hline
\end{tabular}

Lucas N, Macaskill P, Irwig L, Moran R, Bogduk N. Reliability of physical examination for diagnosis of myofascial trigger points. Clin J Pain 2009; 25:80-89 (72).

against the inclusion criteria.

- All articles with possible relevance were then retrieved in full text for comprehensive assessment of internal validity, quality, and adherence to inclusion criteria.

\subsubsection{Inclusion and Exclusion Criteria}

The following are the inclusion and exclusion criteria.

1. Are the patients described in sufficient detail to allow one to decide whether they are comparable to those who are treated in interventional pain management clinical practices?

A. Setting - office, hospital, outpatient, inpatient.

B. Physician - interventional pain physician, general physician, anesthesiologist, physiatrist, neurologist, rheumatologist, orthopedic surgeon, neurosurgeon, etc.

C. Patient characteristics - duration of pain.

D. Noninterventional techniques or surgical intervention in the past.
2. Is the intervention described in sufficient detail to enable one to apply its use to patients in interventional pain management settings?
A. Nature of intervention.
B. Frequency of intervention.
C. Duration of intervention.

3. Were clinically relevant outcomes measured?

A. Proportion of pain relief.

B. Disorder/specific disability.

C. Functional improvement.

D. Allocation of eligible and noneligible patients to return to work.

E. Ability to work.

\subsubsection{Clinical Relevance}

The clinical relevance of the included studies was evaluated according to 5 questions recommended by the Cochrane Back Review Group (Table 2) $(135,136)$. Each question was scored as positive $(+)$ if the clinical relevance item was met, negative $(-)$ if the item was not met, and unclear (?) if data were not available to answer the question. 
Table 2. Clinical relevance questions.

\begin{tabular}{|c|c|c|c|}
\hline & $\mathbf{P}(+)$ & $\mathbf{N}(-)$ & U (unclear) \\
\hline $\begin{array}{l}\text { A) Are the patients described in detail so that one can decide whet } \\
\text { who are treated in clinical practice? }\end{array}$ & & & \\
\hline $\begin{array}{l}\text { B) Are the interventions and treatment settings described in suffic } \\
\text { practice? }\end{array}$ & & & \\
\hline C) Were clinically relevant outcomes measured and reported? & & & \\
\hline D) Is the size of the effect clinically meaningful? & & & \\
\hline E) Do the likely treatment benefits outweigh the potential harms? & & & \\
\hline
\end{tabular}

Scoring adapted and modified from Staal JB, et al. Injection therapy for subacute and chronic low-back pain. Cochrane Database Syst Rev 2008; 3:CD001824 (136).

\subsubsection{Methodological Quality or Validity Assessment}

Each study was evaluated by at least 2 authors for stated criteria and any disagreements discussed with a third reviewer. Authors with a perceived conflict of interest for any manuscript were recused from reviewing the manuscript.

Only diagnostic accuracy studies meeting at least $50 \%$ of applicable inclusion criteria were included for analysis. Studies scoring less than $50 \%$ are reported descriptively with critical analysis.

\subsubsection{Data Extraction and Management}

Two review authors independently, in an unblinded standardized manner, extracted the data from the included studies. Disagreements were resolved by discussion between the 2 reviewers; if no consensus could be reached, a third author was called in to break the impasse.

\subsubsection{Assessment of Heterogeneity}

Whenever meta-analyses were conducted, the Isquared (12) index was used to identify heterogeneity (137). Combined results with $12>50 \%$ were considered substantially heterogenous.

Analysis of the evidence was based on diagnostic criteria as follows: 1) blocks in which the reference standard for diagnosis was between $50 \%$ to $80 \%$ pain relief with a single block; 2 ) blocks in which the reference standard for diagnosis was between $50 \%$ to $80 \%$ pain relief with dual blocks; 3 ) blocks in which the reference standard for diagnosis was between $80 \%$ to $100 \%$ pain relief with a single block; and 4) blocks in which the reference standard for diagnosis was between $80 \%$ to $100 \%$ pain relief with dual blocks, to reduce clinical heterogeneity.

\subsubsection{Measurement of Treatment Effect in Data Synthesis (Meta-Analysis)}

Data were separately summarized using meta-analysis when at least 5 studies per type of diagnostic criteria were available that met the inclusion criteria (e.g., single block, double blocks, and $50 \%$ to $80 \%$ relief. )

The minimum acceptable relief was considered to be $50 \%$; however, data were sub-analyzed for $\geq 80 \%$ and $50 \%$ to $80 \%$ relief as the cutoff threshold for a positive block during the performance of previously painful movements. Four separate diagnostic categories were evaluated (i.e., $50 \%$ to $80 \%$ relief as the cutoff threshold with single and dual blocks; and $80 \%$ to $100 \%$ relief as the cutoff threshold with single or dual blocks). For dual blocks, there had to have been concordant response with short-acting and long-acting local anesthetics, or placebo.

\subsubsection{Integration of Heterogeneity}

A meta-analysis was performed only if there were at least 5 studies meeting inclusion criteria for each variable.

Statistical heterogeneity was explored using univariate meta-regression (137).

\subsection{Summary Measures}

Summary measures included $50 \%$ to $80 \%$ or $80 \%$ to $100 \%$ pain relief with the capability of performing previously painful movements concordant with the duration of local anesthetic.

\subsection{Analysis of Evidence}

The analysis of the evidence was performed based on United States Preventive Services Task Force (USPSTF) criteria (138) as illustrated in Table 3 , which has been utilized by multiple authors $(22,23,27,28,139-147)$. 
Table 3. Method for grading the overall strength of the evidence for an intervention.

\begin{tabular}{|l|l||}
\hline Grade & Definition \\
\hline Good & $\begin{array}{l}\text { Evidence includes consistent results from well-designed, well-conducted studies in representative populations that directly assess } \\
\text { effects on health outcomes (at least 2 consistent, higher-quality RCTs or studies of diagnostic test accuracy). }\end{array}$ \\
\hline Fair & $\begin{array}{l}\text { Evidence is sufficient to determine effects on health outcomes, but the strength of the evidence is limited by the number, quality, } \\
\text { size, or consistency of included studies; generalizability to routine practice; or indirect nature of the evidence on health outcomes } \\
\text { (at least one higher-quality trial or study of diagnostic test accuracy of sufficient sample size; 2 or more higher-quality trials or } \\
\text { studies of diagnostic test accuracy with some inconsistency; at least 2 consistent, lower-quality trials or studies of diagnostic test } \\
\text { accuracy, or multiple consistent observational studies with no significant methodological flaws). }\end{array}$ \\
\hline $\begin{array}{l}\text { Limited } \\
\text { or Poor }\end{array}$ & $\begin{array}{l}\text { Evidence is insufficient to assess effects on health outcomes because of limited number or power of studies, large and unexplained } \\
\text { inconsistency between higher-quality trials, important flaws in trial design or conduct, gaps in the chain of evidence, or lack of } \\
\text { information on important health outcomes. }\end{array}$ \\
\hline
\end{tabular}

Adapted and modified from methods developed by U.S. Preventive Services Task Force $(22,138)$.

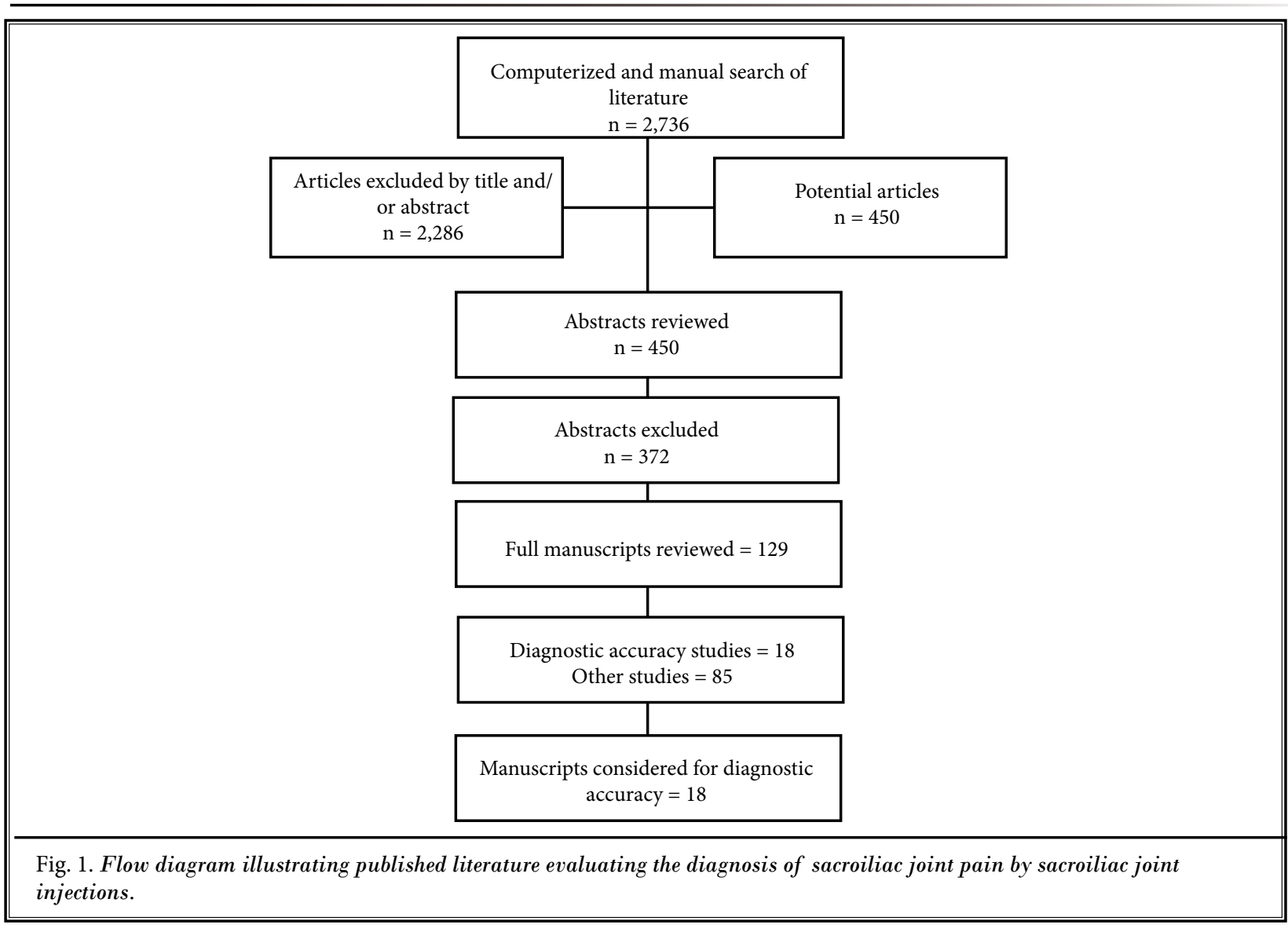

The analysis was conducted using 3 levels of evidence ranging from good to fair to limited (or poor) $(22,23,138)$.

At least 2 of the review authors independently, in an unblinded standardized manner, analyzed the evidence. Any disagreements between reviewers were resolved by a third author and consensus. If there were any conflicts of interest (e.g., authorship), those review- ers were recused from assessment and analysis.

\subsection{Outcome of the Studies}

Outcomes included the prevalence of sacroiliac joint pain and false-positive rate. Based on the above parameters, the reliability of the data derived from each study was assessed.

\subsection{Results}


Figure 1 shows a flow diagram of study selection. There were 103 studies considered for inclusion $\quad(41,42,54,55,71,78,79,82,83,89,119,123,148-237)$ Among these, 18 evaluated diagnostic sacroiliac joint injections $(41,42,54,55,78,79,82,83,119,148$, $152,154,155,157,159,160,175,194,195), \quad 13$ evaluated provocative testing and clinical evaluation
$(54,82,148,154,155,157,159,160,175,189,199-201)$, 43 evaluated diagnostic imaging $(83,152,156,166-$ $168,171,176,202-205,207-237), 4$ evaluated the accuracy of sacroiliac joint injections with fluoroscopy, CT or magnetic resonance imaging (MRI) $(191-193,198)$, and 8 evaluated pain patterns $(82,148,153,161-165)$. Table 4 shows the list of the 23 excluded studies

Table 4. List of excluded studies.

\begin{tabular}{|c|c|}
\hline Manuscript Author(s) & Reason for Exclusion \\
\hline Berthelot et al (89) & This was a review article rather than a diagnostic accuracy study. \\
\hline DePalma et al (123) & $\begin{array}{l}\text { This was a study of patients with or without surgical discectomy with only } 11 \text { patients being included who } \\
\text { had surgical discectomy with } 0 \% \text { prevalence of sacroiliac joint pain in patients with surgical discectomy and } \\
18.1 \% \text { in patients without surgery. }\end{array}$ \\
\hline Maigne et al (156) & $\begin{array}{l}\text { Inclusion criteria was of patients suffering with } 7 \text { weeks of pain pattern compatible with sacroiliac joint pain } \\
\text { - acute pain. }\end{array}$ \\
\hline Klauser et al (169) & $\begin{array}{l}\text { This study evaluated the feasibility of ultrasound-guided sacroiliac joint injection with landmarks at } 2 \\
\text { different levels. }\end{array}$ \\
\hline Harmon \& Alexiev (170) & Sonoanatomy and injection technique of iliolumbar ligament was evaluated. \\
\hline Gupta (172) & $\begin{array}{l}\text { An alternative method with a double needle technique for performing difficult sacroiliac joint injections } \\
\text { was evaluated. }\end{array}$ \\
\hline Hart et al (173) & $\begin{array}{l}\text { Intraarticular injections of the sacroiliac joint were evaluated after lumbar stabilization as a therapeutic } \\
\text { modality. }\end{array}$ \\
\hline Morimoto et al (174) & This was a case description of abdominal pain associated with sacroiliac joint dysfunction. \\
\hline Hamauchi et al (177) & This was a case report presenting acute low back pain secondary to sacroiliac joint arthritis arthropathy. \\
\hline Migliore et al (178) & A technical contribution for ultrasound-guided injection of sacroiliac joints was evaluated. \\
\hline Streitparth et al (179) & $\begin{array}{l}\text { Evaluation included image-guided spinal injection procedures in open high field MRI with vertical field } \\
\text { orientation studying its feasibility and technical features. }\end{array}$ \\
\hline Khurana et al (180) & Therapeutic intervention with percutaneous fusion was evaluated. \\
\hline Dreyfuss et al (181) & $\begin{array}{l}\text { An evaluation of the ability of single site, single depth sacral lateral branch blocks to anesthetize the } \\
\text { sacroiliac joint complex showed significant anatomic limitations with single site, single depth lateral branch } \\
\text { injections rendering them physiologically ineffective on a consistent basis. }\end{array}$ \\
\hline Dreyfuss et al (182) & $\begin{array}{l}\text { The evaluation of the ability of multi-site, multi-depth sacral lateral branch blocks to anesthetize the } \\
\text { sacroiliac joint complex showed that there is physiologic evidence that the intraarticular portion of the } \\
\text { sacroiliac joint is innervated from both ventral and dorsal sources. }\end{array}$ \\
\hline Sadreddini et al (183) & An evaluation of unguided sacroiliac joint injections showing effectiveness. \\
\hline Borowksy \& Fagen (184) & $\begin{array}{l}\text { This study evaluated the sources of sacroiliac region pain to gain insight into intraarticular injection } \\
\text { compared to a combination of intraarticular and periarticular injection rather than determining prevalence. } \\
\text { The prevalence estimates were not available. Only outcomes were available. }\end{array}$ \\
\hline Harmon \& O’Sullivan (185) & Injection technique with ultrasound guidance was evaluated. \\
\hline Günaydin et al (186) & $\begin{array}{l}\text { This study was an evaluation of the therapeutic effectiveness of repeat injections under magnetic resonance } \\
\text { imaging. }\end{array}$ \\
\hline Murakami et al (187) & $\begin{array}{l}\text { This study was a comparative evaluation of periarticular and intraarticular lidocaine injections for sacroiliac } \\
\text { joint pain. }\end{array}$ \\
\hline Haufe \& Mork (188) & This is a description of a technique for the treatment of sacroiliac joint pain with sacroiliac joint debridement. \\
\hline Pekkafahli et al (190) & Sacroiliac joint injections were performed with sonographic guidance. \\
\hline Bokov et al (196) & $\begin{array}{l}\text { An evaluation of the reasons for failed back surgery syndrome and partial results after different types of } \\
\text { surgical lumbar nerve root compression; however, there was no evaluation for sacroiliac joint. }\end{array}$ \\
\hline
\end{tabular}


$(89,123,156,169,170,172-174,177-188,190,196)$.

\subsection{Diagnostic Accuracy Studies}

Table 5 illustrates the characteristics of studies considered for inclusion. There were 3 studies utilizing a single block with $50 \%$ to $80 \%$ relief as the cut- off threshold $(82,83,157), 7$ studies utilizing dual blocks with $50 \%$ to $80 \%$ relief as the cutoff threshold $(54,55,78,79,119,160,194,195)$, and one duplicate study $(78,195)$. There were 6 studies utilizing $80 \%$ to $100 \%$ relief as the cutoff threshold with a single block $(42,148,152,154,155,175)$, and 2 studies utilizing dual

Table 5. Characteristics of reported diagnostic accuracy studies.

\begin{tabular}{|c|c|c|c|c|}
\hline & Participants & Objective(s) & Interventions(s) & Result(s) \\
\hline \multicolumn{5}{|c|}{ 50\% TO 79\% RELIEF WITH SINGLE BLOCK } \\
\hline $\begin{array}{l}\text { Schwarzer et al (82) } \\
\text { Utilized } 75 \% \text { relief }\end{array}$ & $\begin{array}{l}43 \text { consecutive patients with } \\
\text { chronic low back pain maximal } \\
\text { below L5/S1 were investigated. }\end{array}$ & $\begin{array}{l}\text { To establish the prevalence } \\
\text { of sacroiliac joint pain, the } \\
\text { validity of pain provocation, } \\
\text { whether any arthrographic } \\
\text { abnormalities predict a } \\
\text { response to joint block, and } \\
\text { whether certain pain patterns } \\
\text { discriminate patients with this } \\
\text { diagnosis. }\end{array}$ & $\begin{array}{l}\text { Intraarticular injection of } 1 \\
\mathrm{~mL} \text { of } 2 \% \text { lignocaine. }\end{array}$ & $\begin{array}{l}\text { Prevalence }= \\
30 \%\end{array}$ \\
\hline $\begin{array}{l}\text { Maigne \& Planchon (83) } \\
\text { Utilized 75\% relief }\end{array}$ & $\begin{array}{l}\text { This was a prospective series } \\
\text { of } 40 \text { patients with persistent } \\
\text { low back pain after technically } \\
\text { successful fusion who received } \\
\text { a sacroiliac anesthetic block } \\
\text { under fluoroscopic control. }\end{array}$ & $\begin{array}{l}\text { To determine if the sacroiliac } \\
\text { joint could be a possible } \\
\text { source of pain and to search } \\
\text { predictive factors for a positive } \\
\text { block. }\end{array}$ & $\begin{array}{l}\text { Intraarticular injection with } \\
2 \mathrm{~mL} \text { of } 2 \% \text { lidocaine. }\end{array}$ & $\begin{array}{l}\text { Prevalence }= \\
35 \%\end{array}$ \\
\hline $\begin{array}{l}\text { Broadhurst \& Bond (157) } \\
\text { Utilized } 70 \% \text { relief }\end{array}$ & $\begin{array}{l}\text { Double-blind trial of } 40 \text { patients } \\
\text { to determine the sensitivity } \\
\text { and specificity of } 3 \text { commonly } \\
\text { used pain provocation tests } \\
\text { for sacroiliac joint dysfunction } \\
\text { if suppression of pain by } 70 \% \\
\text { with injection of either normal } \\
\text { saline or lidocaine. }\end{array}$ & $\begin{array}{l}\text { To determine the sensitivity } \\
\text { and specificity of } 3 \text { commonly } \\
\text { used pain provocation tests for } \\
\text { sacroiliac joint dysfunction. }\end{array}$ & $\begin{array}{l}\text { Intraarticular injection of } \\
4 \mathrm{~mL} \text { of } 1 \% \text { lignocaine or } 4 \\
\mathrm{~mL} \text { of normal saline into the } \\
\text { painful joint in } 20 \text { patients } \\
\text { in each group. }\end{array}$ & $\begin{array}{l}\text { Prevalence }=\text { not } \\
\text { available }\end{array}$ \\
\hline \multicolumn{5}{|c|}{ 50\% TO 79\% RELIEF WITH DUAL BLOCKS } \\
\hline $\begin{array}{l}\text { Maigne et al (54) } \\
\text { Utilized } 75 \% \text { relief }\end{array}$ & $\begin{array}{l}54 \text { patients aged } 18-75 \text { with } \\
\text { chronic unilateral low back pain } \\
\text { with or without radiation to the } \\
\text { posterior thigh for }>50 \text { days } \\
\text { (median } 4.2 \text { months). Patients } \\
\text { had failed epidural or lumbar } \\
\text { facet injections. }\end{array}$ & $\begin{array}{l}\text { To determine the prevalence } \\
\text { of sacroiliac joint pain in a } \\
\text { selected population of patients } \\
\text { with low back pain and assess } \\
\text { certain pain provocation tests. }\end{array}$ & $\begin{array}{l}\text { Successful blockade of } \\
\text { the sacroiliac joint in } 54 \\
\text { patients. A screening block } \\
\text { was done with } 2 \% \text { lidocaine } \\
\text { and a confirmatory block } \\
\text { was performed with } \\
\text { bupivacaine } 0.5 \% \text {. Greater } \\
\text { than } 75 \% \text { relief was } \\
\text { considered a positive block. }\end{array}$ & $\begin{array}{l}\text { Prevalence }= \\
18.5 \% \\
\text { False-positive } \\
\text { rate }=20 \%\end{array}$ \\
\hline $\begin{array}{l}\text { Irwin et al (55) } \\
\text { Utilized 70\% relief }\end{array}$ & $\begin{array}{l}158 \text { patients underwent } \\
\text { sacroiliac joint injections with } \\
\text { average duration of symptoms } \\
\text { being } 34 \text { months. Patients failed } \\
\text { conservative modalities prior to } \\
\text { injection therapy. }\end{array}$ & $\begin{array}{l}\text { To evaluate the prevalence } \\
\text { and correlation among age, } \\
\text { sex, and body mass index } \\
\text { with dual, comparative local } \\
\text { anesthetic blocks. }\end{array}$ & $\begin{array}{l}\text { The fluoroscopically guided } \\
\text { contrast medium-enhanced } \\
\text { sacroiliac joint injections } \\
\text { were performed initially with } \\
2 \mathrm{~mL} \text { of } 2 \% \text { lidocaine for the } \\
\text { first injection, followed by } \\
2 \mathrm{~mL} \text { of } 0.25 \% \text { bupivacaine, } \\
\text { a local anesthetic, for the } \\
\text { confirmatory injection. A } \\
\text { patient was required to have } \\
\text { at least } 70 \% \text { reduction of } \\
\text { familiar painful symptoms } \\
\text { after the initial injection for } \\
3 \text { or } 4 \text { hours for a positive } \\
\text { response. }\end{array}$ & $\begin{array}{l}\text { Prevalence = } \\
26.6 \% \\
\text { False-positive } \\
\text { rate = Not } \\
\text { available }\end{array}$ \\
\hline
\end{tabular}


Table 5 (cont.). Characteristics of reported diagnostic accuracy studies.

\begin{tabular}{|c|c|c|c|c|}
\hline & Participants & Objective(s) & Interventions(s) & Result(s) \\
\hline $\begin{array}{l}\text { DePalma et al }(78,195) \\
\text { Utilized } 75 \% \text { relief }\end{array}$ & $\begin{array}{l}156 \text { patients underwent } \\
\text { diagnostic procedures } \\
\text { including discography, dual } \\
\text { diagnostic facet joint blocks, } \\
\text { and intraarticular sacroiliac } \\
\text { joint injections to evaluate the } \\
\text { source of chronic low back } \\
\text { pain based on age. A screening } \\
\text { block was performed with } 1 \% \\
\text { lidocaine and a confirmatory } \\
\text { block was performed with } 0.5 \% \\
\text { bupivacaine. }\end{array}$ & $\begin{array}{l}\text { To estimate the prevalence, } \\
\text { mean age, and association of } \\
\text { prevalence and age of lumbar } \\
\text { internal disc disruption, } \\
\text { facet joint pain, sacroiliac } \\
\text { joint pain, spinal and pelvic } \\
\text { insufficiency fractures, } \\
\text { interspinous ligament injury/ } \\
\text { Baastrup Disease, and soft } \\
\text { tissue irritation by fusion } \\
\text { hardware. }\end{array}$ & $\begin{array}{l}\text { Intraarticular sacroiliac } \\
\text { joint injections with } 2 \mathrm{~mL} \\
\text { of } 1 \% \text { lidocaine and } 0.5 \% \\
\text { bupivacaine. }\end{array}$ & $\begin{array}{l}\text { Prevalence }= \\
18.2 \% \\
\\
\text { False-Positive } \\
\text { Rate }=\text { Not } \\
\text { available }\end{array}$ \\
\hline $\begin{array}{l}\text { DePalma et al (79) } \\
\text { Utilized 75\% relief }\end{array}$ & $\begin{array}{l}\text { Retrospective evaluation of } 27 \\
\text { motor vehicle collision-induced } \\
\text { chronic low back pain patients } \\
\text { undergoing multiple types of } \\
\text { diagnostic interventions. }\end{array}$ & $\begin{array}{l}\text { To estimate prevalence rates } \\
\text { of discogenic, facet, and } \\
\text { sacroiliac joint pain, and } \\
\text { describe clinical features of } \\
\text { chronic low back pain patients } \\
\text { whose symptoms were } \\
\text { initiated by motor vehicle } \\
\text { collision. }\end{array}$ & $\begin{array}{l}\text { Intraarticular sacroiliac } \\
\text { joint injections with } 2 \mathrm{~mL} \\
\text { of } 1 \% \text { lidocaine and } 0.5 \% \\
\text { bupivacaine. }\end{array}$ & $\begin{array}{l}\text { Prevalence = } \\
18.2 \% \\
\text { False-Positive } \\
\text { Rate }=\text { Not } \\
\text { available }\end{array}$ \\
\hline $\begin{array}{l}\text { DePalma et al (119) } \\
\text { Utilized } 75 \% \text { relief }\end{array}$ & $\begin{array}{l}\text { The diagnoses of } 28 \text { fusion } \\
\text { cases identified from } 170 \text { low } \\
\text { back pain patients undergoing } \\
\text { diagnostic procedures included } \\
12 \text { with sacroiliac joint pain. }\end{array}$ & $\begin{array}{l}\text { To estimate the prevalence } \\
\text { of lumbar internal disc } \\
\text { disruption, zygapophysial } \\
\text { joint pain, sacroiliac } \\
\text { joint pain, and soft tissue } \\
\text { irritation by fusion hardware } \\
\text { in postfusion low back } \\
\text { pain patients compared to } \\
\text { nonfused patients utilizing } \\
\text { diagnostic spinal procedures. }\end{array}$ & $\begin{array}{l}\text { Intraarticular sacroiliac } \\
\text { joint injections with } 2 \mathrm{~mL} \\
\text { of } 1 \% \text { lidocaine and } 0.5 \% \\
\text { bupivacaine. }\end{array}$ & $\begin{array}{l}\text { Prevalence }= \\
18.2 \% \\
\\
\text { False-Positive } \\
\text { Rate }=\text { Not } \\
\text { available }\end{array}$ \\
\hline $\begin{array}{l}\text { van der Wurff et al (160) } \\
\text { Utilized 50\% relief }\end{array}$ & $\begin{array}{l}\text { Total number of } 140 \text { patients } \\
\text { with chronic low back pain } \\
\text { visiting a pain clinic in the } \\
\text { Netherlands; } 60 \text { patients } \\
\text { entered the study. }\end{array}$ & $\begin{array}{l}\text { To compare the diagnostic } \\
\text { accuracy of a multi-test } \\
\text { regimen of } 5 \text { sacroiliac joint } \\
\text { pain provocation tests with } \\
\text { fluoroscopically controlled } \\
\text { double sacroiliac joint blocks } \\
\text { using a short- and long-acting } \\
\text { local anesthetic. }\end{array}$ & $\begin{array}{l}\text { The fluoroscopically guided } \\
\text { contrast medium-enhanced } \\
\text { sacroiliac joint injections } \\
\text { were performed initially } \\
\text { with } 2 \mathrm{~mL} \text { of } 2 \% \text { lidocaine } \\
\text { and then with } 0.25 \% \\
\text { bupivacaine. } \\
\text { A reduction in the patient's } \\
\text { characteristic pain of } 50 \% \\
\text { or more on the visual } \\
\text { analog scale (VAS) lasting } \\
\text { for at least one hour for } \\
\text { lidocaine or } 4 \text { hours for } \\
\text { bupivacaine was considered } \\
\text { as positive. When a patient } \\
\text { showed a VAS reduction } \\
\text { after both intraarticular } \\
\text { sacroiliac joint blocks, this } \\
\text { was considered a positive } \\
\text { response. Any other } \\
\text { outcome was considered a } \\
\text { negative response. }\end{array}$ & $\begin{array}{l}\text { Prevalence }= \\
38 \% \\
\text { False-positive } \\
\text { rate }=21 \%\end{array}$ \\
\hline
\end{tabular}


Table 5 (cont.). Characteristics of reported diagnostic accuracy studies.

\begin{tabular}{|c|c|c|c|c|}
\hline & Participants & Objective(s) & Interventions(s) & Result(s) \\
\hline Liliang et al (194) & $\begin{array}{l}130 \text { patients who underwent } \\
\text { lumbar or lumbosacral fusion } \\
\text { were evaluated for sacroiliac } \\
\text { joint pain. Of these, } 52 \text { patients } \\
\text { obtained positive findings } \\
\text { with } 3 \text { of the provocative tests } \\
\text { for sacroiliac joint pain. They } \\
\text { were selected to receive dual } \\
\text { diagnostic blocks. Among the } \\
52 \text { patients, } 20 \text { were considered } \\
\text { to have sacroiliac joint pain on } \\
\text { the basis of } 2 \text { positive responses } \\
\text { to diagnostic blocks with } 75 \% \\
\text { as the criterion standard. }\end{array}$ & $\begin{array}{l}\text { To ascertain whether } \\
\text { sacroiliac joint pain represents } \\
\text { a potential source of pain in } \\
\text { patients who have undergone } \\
\text { lumbar or lumbosacral } \\
\text { fusions. }\end{array}$ & $\begin{array}{l}\text { Intraarticular injections } \\
\text { of either lidocaine or } \\
\text { bupivacaine, } 1 \mathrm{~mL} \text {, with } \\
40 \mathrm{mg} \text { of triamcinolone } \\
\text { acetonide. }\end{array}$ & $\begin{array}{l}\text { Prevalence = } \\
40 \% \\
\text { False-positive } \\
\text { rate }=26 \%\end{array}$ \\
\hline \multicolumn{5}{|c|}{$\begin{array}{l}80 \% \text { TO } 100 \% \text { RELIEF } \\
\text { WITH SINGLE BLOCK }\end{array}$} \\
\hline $\begin{array}{l}\text { Pang et al (42) } \\
\text { Utilized } 90 \% \text { relief }\end{array}$ & $\begin{array}{l}104 \text { consecutive adult patients } \\
\text { who underwent spinal pain } \\
\text { mapping were examined and } \\
\text { analyzed. They found in this } \\
\text { group a total of } 87 \% \text { of the } \\
\text { patients with a diagnosed } \\
\text { pain source and } 13 \% \text { without } \\
\text { a source. In this evaluation, } \\
\text { sacroiliac joint pain was } \\
\text { identified in } 10 \% \text { of the patients } \\
\text { from the total sample. }\end{array}$ & $\begin{array}{l}\text { To evaluate the usefulness of } \\
\text { this modality in diagnosing } \\
\text { low back pain of uncertain } \\
\text { etiology. }\end{array}$ & $\begin{array}{l}\text { Intraarticular injection with } \\
2 \mathrm{~mL} \text { of } 2 \% \text { lidocaine. }\end{array}$ & $\begin{array}{l}\text { Prevalence }= \\
10 \% \text { of total } \\
\text { sample }\end{array}$ \\
\hline $\begin{array}{l}\text { Dreyfuss et al (148) } \\
\text { Utilized } 90 \% \text { relief }\end{array}$ & $\begin{array}{l}\text { This prospective study included } \\
85 \text { patients based on historical } \\
\text { data with } 12 \text { tests performed } \\
\text { by } 2 \text { examiners. } 90 \% \text { or more } \\
\text { relief was considered a positive } \\
\text { response, and less than } 90 \% \\
\text { relief was considered a negative } \\
\text { response. }\end{array}$ & $\begin{array}{l}\text { To identify a single sacroiliac } \\
\text { joint test or ensemble of tests } \\
\text { that are sufficiently useful in } \\
\text { diagnosing sacroiliac joint } \\
\text { disorders to be clinically } \\
\text { valuable. }\end{array}$ & $\begin{array}{l}\text { Intraarticular injection of } \\
1.5 \mathrm{~mL} \text { of } 2 \% \text { lignocaine } \\
\text { and } 0.5 \mathrm{~mL} \text { of Celestone } \\
\text { Soluspan (betamethasone) } \\
\text { unless a firm endpoint was } \\
\text { reached before this volume. }\end{array}$ & $\begin{array}{l}\text { Prevalence }= \\
53 \%\end{array}$ \\
\hline $\begin{array}{l}\text { Slipman et al (152) } \\
\text { Utilized } 80 \% \text { relief }\end{array}$ & $\begin{array}{l}50 \text { consecutive patients meeting } \\
\text { a pre-established criteria from a } \\
\text { chronic spine practice. }\end{array}$ & $\begin{array}{l}\text { To determine the sensitivity } \\
\text { and specificity of radionuclide } \\
\text { imaging in establishing a } \\
\text { diagnosis of sacroiliac joint } \\
\text { syndrome in patients with low } \\
\text { back pain. }\end{array}$ & $\begin{array}{l}\text { Intraarticular injection of } \\
1 \mathrm{~mL} \text { of betamethasone } \\
\text { sodium phosphate and } \\
\text { acetate suspension, } 60 \\
\text { mg per mL, } 3 \mathrm{~mL} \text { of } 1 \% \\
\text { lidocaine hydrochloride, } \\
\text { or } 3 \mathrm{~mL} \text { of } 2 \% \text { lidocaine } \\
\text { hydrochloride. Among } \\
\text { the patients with positive } \\
\text { response, there were } 27 \\
\text { patients with negative scans } \\
\text { and } 4 \text { patients with positive } \\
\text { scans. }\end{array}$ & $\begin{array}{l}\text { Prevalence }= \\
62 \%\end{array}$ \\
\hline $\begin{array}{l}\text { Laslett et al (154) } \\
\text { Utilized } 80 \% \text { relief }\end{array}$ & $\begin{array}{l}\text { Prospective evaluation of } 48 \\
\text { patients satisfying inclusion } \\
\text { criteria from a total of } 62 \\
\text { patients agreeing to participate } \\
\text { and were evaluated. Patients } \\
\text { with buttock pain, with or } \\
\text { without lumbar or lower } \\
\text { extremity symptoms were } \\
\text { included. }\end{array}$ & $\begin{array}{l}\text { To examine the diagnostic } \\
\text { power of pain provocation } \\
\text { sacroiliac joint tests singly } \\
\text { and in various combinations } \\
\text { in relation to an accepted } \\
\text { criterion standard. }\end{array}$ & $\begin{array}{l}\text { Intraarticular injection } \\
\text { of } 1 \mathrm{~mL} \text { of } 2 \% \text { lignocaine. } \\
\text { All patients underwent } \\
\text { provocation testing. }\end{array}$ & $\begin{array}{l}\text { Prevalence }= \\
33 \%\end{array}$ \\
\hline
\end{tabular}


Table 5 (cont.). Characteristics of reported diagnostic accuracy studies.

\begin{tabular}{|c|c|c|c|c|}
\hline & Participants & Objective(s) & Interventions(s) & Result(s) \\
\hline $\begin{array}{l}\text { Young et al (155) } \\
\text { Utilized } 80 \% \text { relief }\end{array}$ & $\begin{array}{l}\text { A prospective evaluation } \\
\text { of } 81 \text { patients with chronic } \\
\text { lumbopelvic pain to evaluate } \\
\text { the correlation of the clinical } \\
\text { examination characteristics } \\
\text { with } 3 \text { sources of chronic low } \\
\text { back pain with diagnostic } \\
\text { injections as criterion standard. } \\
57 \text { patients were suspected to } \\
\text { have sacroiliac joint pain. }\end{array}$ & $\begin{array}{l}\text { To identify significant } \\
\text { components of a clinical } \\
\text { examination that are } \\
\text { associated with symptomatic } \\
\text { lumbar discs, zygapophysial } \\
\text { joints, and sacroiliac joints. }\end{array}$ & $\begin{array}{l}\text { Intraarticular injection with } \\
1.5 \mathrm{~mL} \text { of lidocaine. }\end{array}$ & $\begin{array}{l}\text { Prevalence = } \\
39 \%\end{array}$ \\
\hline $\begin{array}{l}\text { Stanford \& Burnham } \\
\text { (175) } \\
\text { Utilized } 80 \% \text { relief }\end{array}$ & $\begin{array}{l}\text { Evaluation of } 34 \text { patients with } \\
\text { suspected unilateral mechanical } \\
\text { sacroiliac joint pain. }\end{array}$ & $\begin{array}{l}\text { To evaluate the diagnostic } \\
\text { usefulness of repeating } \\
\text { sacroiliac joint provocative } \\
\text { tests postblock. }\end{array}$ & $\begin{array}{l}\text { Intraarticular injection of } \\
1.5 \mathrm{~mL} \text { of } 2 \% \text { lidocaine and } \\
1 \mathrm{~mL} \text { of corticosteroid. }\end{array}$ & $\begin{array}{l}\text { Prevalence }= \\
32 \%\end{array}$ \\
\hline \multicolumn{5}{|l|}{$\begin{array}{l}80 \% \text { TO } 100 \% \text { RELIEF } \\
\text { WITH DUAL BLOCKS }\end{array}$} \\
\hline $\begin{array}{l}\text { Manchikanti et al (41) } \\
\text { Utilized } 80 \% \text { relief }\end{array}$ & $\begin{array}{l}120 \text { patients (age 18-90) } \\
\text { presenting to the clinic with > } \\
6 \text { months of low back pain and } \\
\text { no structural basis for the pain } \\
\text { by radiographic imaging. } 20 \\
\text { patients were evaluated for SI } \\
\text { joint pain. }\end{array}$ & $\begin{array}{l}\text { To determine the frequency of } \\
\text { various structures responsible } \\
\text { for low back pain. }\end{array}$ & $\begin{array}{l}\text { All patients had facet } \\
\text { blocks. } \\
\text { Those not responding who } \\
\text { fit the criteria had double } \\
\text { injection sacroiliac joint } \\
\text { blocks. The screening block } \\
\text { was done with } 2 \% \text { lidocaine } \\
\text { and the confirmatory block } \\
\text { was performed using } 0.5 \% \\
\text { bupivacaine. }\end{array}$ & $\begin{array}{l}\text { Prevalence = } \\
10 \% \\
\text { False-positive } \\
\text { rate }=22 \%\end{array}$ \\
\hline $\begin{array}{l}\text { Laslett et al (159) } \\
\text { Utilized 80\% relief }\end{array}$ & $\begin{array}{l}48 \text { patients received an initial } \\
\text { sacroiliac joint diagnostic } \\
\text { injection, derived from } 62 \\
\text { patients with buttock pain with } \\
\text { or without lumbar or lower } \\
\text { extremity symptoms. }\end{array}$ & $\begin{array}{l}\text { To assess the diagnostic } \\
\text { accuracy of clinical } \\
\text { examination in identifying } \\
\text { symptomatic and } \\
\text { asymptomatic sacroiliac joints } \\
\text { using double-diagnostic } \\
\text { injections as the reference } \\
\text { standard. }\end{array}$ & $\begin{array}{l}16 \text { patients had a positive } \\
\text { response to sacroiliac joint } \\
\text { injections and } 5 \text { of them did } \\
\text { not receive a confirmatory } \\
\text { diagnostic injection } \\
\text { because they derived such } \\
\text { symptomatic relief from } \\
\text { the initial procedure that } \\
\text { a confirmatory injection } \\
\text { could not be justified. } \\
11 \text { patients received a } \\
\text { confirmatory injection and } \\
\text { all of them tested positive. } \\
\text { Overall } 32 \text { patients had } \\
\text { negative sacroiliac joint } \\
\text { injections and did not } \\
\text { require a confirmatory } \\
\text { injection. }\end{array}$ & $\begin{array}{l}\text { Prevalence = } \\
25.6 \% \\
\text { False-positive } \\
\text { rate = NA }\end{array}$ \\
\hline
\end{tabular}


blocks with a $>80 \%$ cutoff threshold $(41,159)$.

\subsubsection{Clinical Relevance}

Among the 18 studies assessed for clinical relevance $\quad(41,42,54,55,78,79,82,83,119,148,152,154,155$ $, 157,159,160,175,194,195)$ with one duplicate publication $(78,195), 17$ studies met criteria, scoring 5 out of 5 $(41,42,54,55,78,79,82,83,119,148,152,154,155,159,160$, $175,194,195)$. Table 6 illustrates the assessment of clinical relevance.

\subsubsection{Methodological Quality Assessment}

A methodological quality assessment of diagnostic accuracy studies meeting inclusion criteria was carried out utilizing QAREL criteria as shown in Table 7. Studies achieving $50 \%$ or higher scores were included. Scores of $67 \%$ or higher were considered to be high quality, $50 \%$ were considered to be moderate quality, and stud- ies scoring less than $50 \%$ were considered to be of poor quality and excluded.

There were 3 studies utilizing a single block with $50 \%$ to $80 \%$ relief as the cutoff threshold $(82,83,157)$, 7 studies utilizing $50 \%$ to $80 \%$ relief following dual blocks $(54,55,78,79,119,160,194,195)$, with one duplicate study $(78,195)$. There were 6 studies utilizing $>80 \%$ relief following a single block as the reference standard $(42,148,152,154,155,175)$, and 2 studies in which $>80 \%$ relief following dual blocks was used as the diagnostic criterion $(41,159)$.

There were 18 studies evaluating diagnostic accuracy $\quad(41,42,54,55,78,79,82,83,119,148,152,154$, $155,157,159,160,175,194,195)$, with one study being published in duplicate $(78,195)$. Seventeen studies were considered to be high quality $(41,42,54,55,78$, $79,82,83,119,148,152,154,155,157,159,160,194,195)$

Table 6. Clinical relevance of included studies.

\begin{tabular}{|c|c|c|c|c|c|c|}
\hline Manuscript Author(s) & $\begin{array}{l}\text { A) Patient } \\
\text { description }\end{array}$ & $\begin{array}{l}\text { B) Description } \\
\text { of interventions } \\
\text { and treatment } \\
\text { settings }\end{array}$ & $\begin{array}{l}\text { C) Clinically } \\
\text { relevant } \\
\text { outcomes }\end{array}$ & $\begin{array}{l}\text { D) Clinical } \\
\text { importance }\end{array}$ & $\begin{array}{l}\text { E) Benefits } \\
\text { versus } \\
\text { potential } \\
\text { harms }\end{array}$ & $\begin{array}{l}\text { Total } \\
\text { Criteria } \\
\text { Met }\end{array}$ \\
\hline Manchikanti et al (41) & + & + & + & + & + & $5 / 5$ \\
\hline Pang et al (42) & + & + & + & + & + & $5 / 5$ \\
\hline Maigne et al (54) & + & + & + & + & + & $5 / 5$ \\
\hline Irwin et al (55) & + & + & + & + & + & $5 / 5$ \\
\hline DePalma et al $(78,195)$ & + & + & + & + & + & $5 / 5$ \\
\hline DePalma et al (79) & + & + & + & + & + & $5 / 5$ \\
\hline Schwarzer et al (82) & + & + & + & + & + & $5 / 5$ \\
\hline Maigne \& Planchon (83) & + & + & + & + & + & $5 / 5$ \\
\hline DePalma et al (119) & + & + & + & + & + & $5 / 5$ \\
\hline Dreyfuss et al (148) & + & + & + & + & + & $5 / 5$ \\
\hline Slipman et al (152) & + & + & + & + & + & $5 / 5$ \\
\hline Laslett et al (154) & + & + & + & + & + & $5 / 5$ \\
\hline Young et al (155) & + & + & + & + & + & $5 / 5$ \\
\hline Broadhurst \& Bond (157) & + & - & - & - & + & $2 / 5$ \\
\hline Laslett et al (159) & + & + & + & + & + & $5 / 5$ \\
\hline van der Wurff et al (160) & + & + & + & + & + & $5 / 5$ \\
\hline Stanford \& Burnham (175) & + & + & + & + & + & $5 / 5$ \\
\hline Liliang et al (194) & + & + & + & + & + & $5 / 5$ \\
\hline
\end{tabular}

$+=$ positive; - = negative

Scoring adapted from Staal JB, et al. Injection therapy for subacute and chronic low back pain. Cochrane Database Syst Rev 2008; 3:CD001824 (136). 
Table 7. Quality Appraisal of Diagnostic Reliability checklist.

\begin{tabular}{|c|c|c|c|c|c|c|c|c|c|c|c|}
\hline & $\begin{array}{l}\text { Manchikanti } \\
\text { et al (41) }\end{array}$ & $\begin{array}{l}\text { Pang et } \\
\text { al (42) }\end{array}$ & $\begin{array}{l}\text { Maigne } \\
\text { et al } \\
(54)\end{array}$ & $\begin{array}{c}\text { Irwin } \\
\text { et al } \\
(55)\end{array}$ & $\begin{array}{c}\text { DePalma } \\
\text { et al } \\
(78,195)\end{array}$ & $\begin{array}{l}\text { DePalma } \\
\text { et al (79) }\end{array}$ & $\begin{array}{c}\text { DePalma } \\
\text { et al } \\
(119)\end{array}$ & $\begin{array}{l}\text { van der } \\
\text { Wurff } \\
\text { et al } \\
(160) \\
\end{array}$ & $\begin{array}{l}\text { Schwarzer } \\
\text { et al (81) }\end{array}$ & $\begin{array}{c}\text { Maigne } \\
\& \\
\text { Planchon } \\
(83) \\
\end{array}$ & $\begin{array}{c}\text { Dreyfuss } \\
\text { et al } \\
(148)\end{array}$ \\
\hline $\begin{array}{l}\text { 1. Was the test evaluated } \\
\text { in a spectrum of } \\
\text { subjects representative } \\
\text { of patients who would } \\
\text { normally receive the test } \\
\text { in clinical practice? }\end{array}$ & $\mathrm{Y}$ & $\mathrm{Y}$ & $\mathrm{Y}$ & $\mathrm{Y}$ & $\mathrm{Y}$ & $\mathrm{Y}$ & $Y$ & $\mathrm{Y}$ & $\mathrm{Y}$ & $\mathrm{Y}$ & Y \\
\hline $\begin{array}{l}\text { 2. Was the test } \\
\text { performed by examiners } \\
\text { representative of those } \\
\text { who would normally } \\
\text { perform the test in } \\
\text { practice? }\end{array}$ & $\mathrm{Y}$ & $\mathrm{Y}$ & $\mathrm{Y}$ & $\mathrm{Y}$ & $\mathrm{Y}$ & $\mathrm{Y}$ & $\mathrm{Y}$ & $\mathrm{Y}$ & $\mathrm{Y}$ & $\mathrm{Y}$ & $\mathrm{Y}$ \\
\hline $\begin{array}{l}\text { 3. Were raters blinded to } \\
\text { the reference standard } \\
\text { for the target disorder } \\
\text { being evaluated? }\end{array}$ & NA & NA & NA & NA & NA & NA & NA & NA & NA & NA & NA \\
\hline $\begin{array}{l}\text { 4. Were raters blinded } \\
\text { to the findings of other } \\
\text { raters during the study? }\end{array}$ & $\mathrm{Y}$ & $\mathrm{Y}$ & $\mathrm{Y}$ & $\mathrm{Y}$ & $\mathrm{Y}$ & $\mathrm{Y}$ & $\mathrm{Y}$ & $\mathrm{Y}$ & $\mathrm{Y}$ & $\mathrm{Y}$ & $\mathrm{Y}$ \\
\hline $\begin{array}{l}\text { 5. Were raters blinded } \\
\text { to their own prior } \\
\text { outcomes of the test } \\
\text { under evaluation? }\end{array}$ & $\mathrm{Y}$ & $\mathrm{Y}$ & $\mathrm{Y}$ & $\mathrm{Y}$ & $\mathrm{Y}$ & $\mathrm{Y}$ & $\mathrm{Y}$ & $\mathrm{Y}$ & $\mathrm{Y}$ & $\mathrm{Y}$ & $\mathrm{Y}$ \\
\hline $\begin{array}{l}\text { 6. Were raters blinded to } \\
\text { clinical information that } \\
\text { may have influenced the } \\
\text { test outcome? }\end{array}$ & $\mathrm{N}$ & $\mathrm{N}$ & $\mathrm{N}$ & $\mathrm{N}$ & $\mathrm{N}$ & $\mathrm{N}$ & $\mathrm{N}$ & $\mathrm{N}$ & $\mathrm{U}$ & $\mathrm{U}$ & $\mathrm{N}$ \\
\hline $\begin{array}{l}\text { 7. Were raters blinded } \\
\text { to additional cues, not } \\
\text { intended to form part } \\
\text { of the diagnostic test } \\
\text { procedure? }\end{array}$ & $\mathrm{N}$ & $\mathrm{N}$ & $\mathrm{N}$ & $\mathrm{N}$ & $\mathrm{N}$ & $\mathrm{N}$ & $\mathrm{N}$ & $\mathrm{N}$ & $\mathrm{N}$ & $\mathrm{N}$ & $\mathrm{N}$ \\
\hline $\begin{array}{l}\text { 8. Was the order in } \\
\text { which raters examined } \\
\text { subjects varied? }\end{array}$ & $\mathrm{Y}$ & $\mathrm{N}$ & $\mathrm{U}$ & $\mathrm{N}$ & $\mathrm{N}$ & $\mathrm{N}$ & $\mathrm{N}$ & $\mathrm{Y}$ & $\mathrm{Y}$ & $\mathrm{U}$ & $\mathrm{U}$ \\
\hline $\begin{array}{l}\text { 9. Were appropriate } \\
\text { statistical measures of } \\
\text { agreement used? }\end{array}$ & $\mathrm{Y}$ & $\mathrm{Y}$ & $\mathrm{Y}$ & $\mathrm{Y}$ & $\mathrm{Y}$ & $\mathrm{Y}$ & $\mathrm{Y}$ & $\mathrm{Y}$ & $\mathrm{Y}$ & $\mathrm{Y}$ & Y \\
\hline $\begin{array}{l}\text { 10. Was the application } \\
\text { and interpretation of } \\
\text { the test appropriate? }\end{array}$ & $\mathrm{Y}$ & $\mathrm{Y}$ & $\mathrm{Y}$ & $\mathrm{Y}$ & $\mathrm{Y}$ & $\mathrm{Y}$ & $\mathrm{Y}$ & $\mathrm{Y}$ & $\mathrm{Y}$ & $\mathrm{Y}$ & $\mathrm{Y}$ \\
\hline $\begin{array}{l}\text { 11. Was the time interval } \\
\text { between measurements } \\
\text { suitable in relation to the } \\
\text { stability of the variable } \\
\text { being measured? }\end{array}$ & $\mathrm{Y}$ & $\mathrm{Y}$ & $\mathrm{Y}$ & $\mathrm{Y}$ & $\mathrm{Y}$ & $\mathrm{Y}$ & $\mathrm{Y}$ & $\mathrm{Y}$ & $\mathrm{Y}$ & $\mathrm{Y}$ & $\mathrm{Y}$ \\
\hline $\begin{array}{l}\text { 12. If there were } \\
\text { dropouts from the } \\
\text { study, was this less than } \\
20 \% \text { of the sample. }\end{array}$ & $\mathrm{Y}$ & $\mathrm{Y}$ & $\mathrm{Y}$ & $\mathrm{Y}$ & $\mathrm{Y}$ & $\mathrm{Y}$ & $\mathrm{Y}$ & $\mathrm{Y}$ & $\mathrm{Y}$ & $\mathrm{Y}$ & $\mathrm{Y}$ \\
\hline TOTAL & $9 / 11$ & $8 / 11$ & $8 / 11$ & $8 / 11$ & $8 / 11$ & $8 / 11$ & $8 / 11$ & $9 / 11$ & $9 / 11$ & $8 / 11$ & $8 / 11$ \\
\hline
\end{tabular}

Y=yes; $\mathrm{N}=$ no; $\mathrm{U}=$ unclear; $\mathrm{N} / \mathrm{A}=$ not applicable

Lucas N, Macaskill P, Irwig L, Moran R, Bogduk N. Reliability of physical examination for diagnosis of myofascial trigger points. Clin J Pain 2009; 25:80-89 (72). 
Table 7 (cont.). Quality Appraisal of Diagnostic Reliability checklist.

\begin{tabular}{|c|c|c|c|c|c|c|c|}
\hline & $\begin{array}{l}\text { Slipman et } \\
\text { al (152) }\end{array}$ & $\begin{array}{l}\text { Laslett et al } \\
(154)\end{array}$ & $\begin{array}{l}\text { Young et al } \\
(155)\end{array}$ & $\begin{array}{l}\text { Broadhurst } \\
\text { \& Bond } \\
(157)\end{array}$ & $\begin{array}{l}\text { Stanford \& } \\
\text { Burnham } \\
(175)\end{array}$ & $\begin{array}{l}\text { Laslett et } \\
\text { al (159) }\end{array}$ & $\begin{array}{l}\text { Liliang } \\
\text { et al } \\
(194)\end{array}$ \\
\hline $\begin{array}{l}\text { 1. Was the test evaluated in a spectrum of subjects } \\
\text { representative of patients who would normally } \\
\text { receive the test in clinical practice? }\end{array}$ & $\mathrm{Y}$ & $\mathrm{Y}$ & $\mathrm{Y}$ & $\mathrm{Y}$ & $\mathrm{Y}$ & $\mathrm{Y}$ & $\mathrm{Y}$ \\
\hline $\begin{array}{l}\text { 2. Was the test performed by examiners } \\
\text { representative of those who would normally perform } \\
\text { the test in practice? }\end{array}$ & $\mathrm{Y}$ & $\mathrm{Y}$ & $\mathrm{Y}$ & $\mathrm{Y}$ & $\mathrm{Y}$ & $\mathrm{Y}$ & Y \\
\hline $\begin{array}{l}\text { 3. Were raters blinded to the reference standard for } \\
\text { the target disorder being evaluated? }\end{array}$ & NA & NA & NA & NA & NA & NA & NA \\
\hline $\begin{array}{l}\text { 4. Were raters blinded to the findings of other raters } \\
\text { during the study? }\end{array}$ & $\mathrm{Y}$ & $\mathrm{Y}$ & $\mathrm{Y}$ & $\mathrm{Y}$ & $\mathrm{U}$ & $\mathrm{Y}$ & $\mathrm{Y}$ \\
\hline $\begin{array}{l}\text { 5. Were raters blinded to their own prior outcomes of } \\
\text { the test under evaluation? }\end{array}$ & $\mathrm{Y}$ & $\mathrm{Y}$ & $\mathrm{Y}$ & $\mathrm{Y}$ & NA & $\mathrm{Y}$ & Y \\
\hline $\begin{array}{l}\text { 6. Were raters blinded to clinical information that may } \\
\text { have influenced the test outcome? }\end{array}$ & $\mathrm{N}$ & $\mathrm{N}$ & $\mathrm{N}$ & $\mathrm{Y}$ & $\mathrm{N}$ & $\mathrm{N}$ & $\mathrm{N}$ \\
\hline $\begin{array}{l}\text { 7. Were raters blinded to additional cues, not } \\
\text { intended to form part of the diagnostic test } \\
\text { procedure? }\end{array}$ & $\mathrm{N}$ & $\mathrm{N}$ & $\mathrm{N}$ & $\mathrm{Y}$ & $\mathrm{N}$ & $\mathrm{N}$ & $\mathrm{N}$ \\
\hline $\begin{array}{l}\text { 8. Was the order in which raters examined subjects } \\
\text { varied? }\end{array}$ & $\mathrm{U}$ & $\mathrm{N}$ & $\mathrm{N}$ & $\mathrm{Y}$ & $\mathrm{N}$ & $\mathrm{N}$ & $\mathrm{N}$ \\
\hline $\begin{array}{l}\text { 9. Were appropriate statistical measures of agreement } \\
\text { used? }\end{array}$ & $\mathrm{Y}$ & $\mathrm{Y}$ & $\mathrm{Y}$ & $\mathrm{Y}$ & $\mathrm{Y}$ & $\mathrm{Y}$ & Y \\
\hline $\begin{array}{l}\text { 10. Was the application and interpretation of the test } \\
\text { appropriate? }\end{array}$ & $\mathrm{Y}$ & $\mathrm{Y}$ & $\mathrm{Y}$ & $\mathrm{Y}$ & $\mathrm{Y}$ & $\mathrm{Y}$ & $\mathrm{Y}$ \\
\hline $\begin{array}{l}\text { 11. Was the time interval between measurements } \\
\text { suitable in relation to the stability of the variable } \\
\text { being measured? }\end{array}$ & $\mathrm{Y}$ & $\mathrm{Y}$ & $\mathrm{Y}$ & $\mathrm{Y}$ & $\mathrm{Y}$ & $\mathrm{Y}$ & Y \\
\hline $\begin{array}{l}\text { 12. If there were dropouts from the study, was this } \\
\text { less than } 20 \% \text { of the sample. }\end{array}$ & $\mathrm{Y}$ & $\mathrm{Y}$ & $\mathrm{Y}$ & $\mathrm{Y}$ & $\mathrm{Y}$ & $\mathrm{Y}$ & $\mathrm{Y}$ \\
\hline TOTAL & $8 / 11$ & $8 / 11$ & $8 / 11$ & $11 / 11$ & $6 / 11$ & $8 / 11$ & $8 / 11$ \\
\hline
\end{tabular}

Y=yes; $\mathrm{N}=$ no; $\mathrm{U}=$ unclear; $\mathrm{N} / \mathrm{A}=$ not applicable

Lucas N, Macaskill P, Irwig L, Moran R, Bogduk N. Reliability of physical examination for diagnosis of myofascial trigger points. Clin J Pain 2009; 25:80-89 (72).

and one study was rated as being of moderate quality (175).

\subsubsection{Meta-Analysis}

All diagnostic accuracy studies were evaluated for homogeneity for inclusion in the meta-analysis (Table 8). There was only one placebo-controlled study (157). There were 7 studies in the dual block group using $50 \%$ to $80 \%$ relief as the cutoff threshold $(54,55,78,79,119,160,194,195)$, with one duplicate study $(78,195)$. Six studies met inclusion that utilized a single block with a cutoff threshold $>80 \%$ pain relief $(42,148,152,154,155,175)$. In the single block stud- ies with a cutoff threshold between $50 \%$ and $80 \%, 2$ studies utilized $75 \%$ pain relief as the criterion standard $(82,83)$ and one study utilized $70 \%$ relief $(157)$. In the studies with a cutoff between $50 \%$ and $80 \%$ pain relief that employed dual blocks as the criterion standard, 3 studies utilized $75 \%$ pain relief as the criterion threshold $(54,78,79,119,195,195)$; with 4 of these publications $(78,79,119,195)$ from one retrospective study $(78)$. Another retrospective evaluation utilized $70 \%$ pain relief as the criterion standard (55), and one prospective study used $50 \%$ relief following double comparative blocks as the criterion standard (160). Thus, the studies 
were not homogenous.

A second category was comprised of 6 studies using a single block with a criterion standard ranging between $80 \%$ and $100 \%$ relief. In this evaluation, 2 studies utilized $90 \%$ pain relief $(42,148)$, whereas 4 studies utilized $80 \%$ or greater relief as criterion standard $(152,154,155,175)$. Inclusion criteria were different. Thus, there was no homogeneity among the studies. In the double block group using a cutoff threshold between $80 \%$ and $100 \%$ pain relief, only 2 studies were identified $(41,159)$.

Consequently, there was no meta-analysis performed.

\subsubsection{Study Characteristics}

Table 9 illustrates the characteristics of the included studies utilizing cutoff thresholds between $50 \%$ and $80 \%$ pain relief, and $>80 \%$ relief, following single and dual blocks.

\subsubsection{Analysis of Evidence}

The evidence was synthesized based on the relief criteria when sacroiliac joint injections were performed. Table 9 illustrates the results of diagnostic studies.

\subsubsection{Single Block with $\mathbf{5 0} \%$ to $\mathbf{8 0} \%$ Pain Relief} Two of the studies evaluating the prevalence

Table 8. Data of prevalence of sacroiliac joint pain by controlled diagnostic blocks.

\begin{tabular}{|c|c|c|c|c|c|}
\hline Study & $\begin{array}{c}\% \text { Relief } \\
\text { Used }\end{array}$ & $\begin{array}{c}\text { Methodological } \\
\text { Criteria Score }\end{array}$ & $\begin{array}{c}\text { Number of } \\
\text { Subjects }\end{array}$ & $\begin{array}{c}\text { Prevalence } \\
\text { Estimates }\end{array}$ & $\begin{array}{c}\text { False-Positive } \\
\text { Rate }\end{array}$ \\
\hline \multicolumn{6}{|c|}{ 50\%-79\% RELIEF WITH A SINGLE BLOCK } \\
\hline Schwarzer et al (82) & $75 \%$ & $9 / 11$ & 43 & $30 \%$ & --- \\
\hline Maigne \& Planchon (83) & $75 \%$ & $8 / 11$ & 40 & $35 \%$ & --- \\
\hline Broadhurst \& Bond (157) & $70 \%$ & $11 / 11$ & 40 & NA & --- \\
\hline \multicolumn{6}{|c|}{ 50\%-79\% RELIEF WITH A DUAL BLOCK } \\
\hline Maigne et al (54) & $75 \%$ & $8 / 11$ & 54 & $18.5 \%$ & $20 \%$ \\
\hline Irwin et al (55) & $70 \%$ & $8 / 11$ & 158 & $26.6 \%$ & NA \\
\hline DePalma et al $(78,195)$ & $75 \%$ & $8 / 11$ & 156 & $18.2 \%$ & NA \\
\hline DePalma et al (79) & $75 \%$ & $8 / 11$ & 27 & $18.2 \%$ & NA \\
\hline DePalma et al (119) & $75 \%$ & $8 / 11$ & 170 & $18.2 \%$ & NA \\
\hline van der Wurff et al (160) & $50 \%$ & $9 / 11$ & 60 & $38 \%$ & $21 \%$ \\
\hline Liliang et al (194) & $75 \%$ & $8 / 11$ & 52 & $40.4 \%$ & $26 \%$ \\
\hline \multicolumn{6}{|c|}{ 80\%-100\% RELIEF WITH A SINGLE BLOCK } \\
\hline Pang et al (42) & $90 \%$ & $8 / 11$ & 104 & $10 \%$ & --- \\
\hline Dreyfuss et al (148) & $90 \%$ & $8 / 11$ & 85 & $53 \%$ & --- \\
\hline Slipman et al (152) & $80 \%$ & $8 / 11$ & 50 & $62 \%$ & --- \\
\hline Laslett et al (154) & $80 \%$ & $8 / 11$ & 48 & $33 \%$ & --- \\
\hline Young et al (155) & $80 \%$ & $8 / 11$ & 81 & $39 \%$ & --- \\
\hline Stanford \& Burnham (175) & $80 \%$ & $6 / 11$ & 34 & $32 \%$ & --- \\
\hline \multicolumn{6}{|c|}{ 80\%-100\% RELIEF WITH DUAL BLOCKS } \\
\hline Manchikanti et al (41) & $80 \%$ & $9 / 11$ & 20 & $10 \%$ & $22 \%$ \\
\hline Laslett et al (159) & $80 \%$ & $8 / 11$ & $43 / 48$ & $25.6 \%$ & NA \\
\hline
\end{tabular}

$\mathrm{NA}=$ Not available 
Prevalence and Diagnostic Accuracy of Sacroiliac Joint Interventions

Table 9. Summary characteristics of studies utilizing $50 \%$ and $70 \%$ relief for single and dual blocks.

\begin{tabular}{|c|c|c|c|c|c|c|c|}
\hline $\begin{array}{c}\text { Reference, } \\
\text { Year }\end{array}$ & $\begin{array}{l}\text { Number of } \\
\text { Patients } \\
\text { Selection } \\
\text { Criteria }\end{array}$ & Intervention & $\begin{array}{l}\text { Outcome } \\
\text { Measures }\end{array}$ & Results & $\begin{array}{c}\text { Strengths } \\
\text { Weaknesses }\end{array}$ & $\begin{array}{l}\text { Methodological } \\
\text { Quality } \\
\text { Assessment } \\
\text { Score }\end{array}$ & Comments \\
\hline \multicolumn{8}{|c|}{ 50\%-79\% RELIEF WITH SINGLE BLOCK } \\
\hline $\begin{array}{l}\text { Schwarzer } \\
\text { et al (82) }\end{array}$ & $\begin{array}{l}43 \text { consecutive } \\
\text { patients with } \\
\text { chronic low } \\
\text { back pain } \\
\text { maximal below } \\
\text { L5/S1 were } \\
\text { investigated. }\end{array}$ & $\begin{array}{l}\text { Intraarticular } \\
\text { injection of } \\
1 \mathrm{~mL} \text { of } 2 \% \\
\text { lignocaine. }\end{array}$ & $\begin{array}{l}\text { A positive } \\
\text { response } \\
\text { was classed } \\
\text { as definite if } \\
\text { there was a } \\
75 \% \text { or greater } \\
\text { reduction of } \\
\text { pain over the } \\
\text { sacroiliac joint } \\
\text { and buttock. }\end{array}$ & $\begin{array}{l}\text { Prevalence }= \\
30 \%\end{array}$ & $\begin{array}{l}\text { Strengths: This study } \\
\text { from } 1995 \text { is the } \\
\text { earliest performed, } \\
\text { with strict selection } \\
\text { criteria and performed } \\
\text { by experts in the field. } \\
\text { Weakness: A single } \\
\text { block. }\end{array}$ & $9 / 11$ & $\begin{array}{l}\text { Well performed } \\
\text { study, but with } \\
\text { a single block } \\
\text { which may } \\
\text { result in lesser } \\
\text { prevalence with } \\
\text { a certain false- } \\
\text { positive rate with } \\
\text { dual blocks. }\end{array}$ \\
\hline $\begin{array}{l}\text { Maigne \& } \\
\text { Planchon } \\
(83)\end{array}$ & $\begin{array}{l}\text { This was a } \\
\text { prospective } \\
\text { series of } 40 \\
\text { patients with } \\
\text { persistent low } \\
\text { back pain after } \\
\text { technically } \\
\text { successful } \\
\text { fusion who } \\
\text { received a } \\
\text { sacroiliac } \\
\text { anesthetic } \\
\text { block under } \\
\text { fluoroscopic } \\
\text { control. }\end{array}$ & $\begin{array}{l}\text { Intraarticular } \\
\text { injection with } \\
2 \mathrm{~mL} \text { of } 2 \% \\
\text { lidocaine. }\end{array}$ & $\begin{array}{l}\text { The study was } \\
\text { performed } \\
\text { appropriately } \\
\text { in patients } \\
\text { after lumbar } \\
\text { fusion. }\end{array}$ & $\begin{array}{l}\text { Prevalence }= \\
35 \%\end{array}$ & $\begin{array}{l}\text { Strengths: The study } \\
\text { was performed } \\
\text { appropriately in } \\
\text { patients after lumbar } \\
\text { fusion. } \\
\text { Weaknesses: There } \\
\text { were } 5 \text { unsuccessful } \\
\text { blocks out of } 45 \\
\text { patients indicating a } \\
\text { high number. Further, } \\
\text { the study evaluated } \\
\text { only the patients with } \\
\text { postfusion pain with a } \\
\text { single block with } 75 \% \\
\text { pain relief. }\end{array}$ & $8 / 11$ & $\begin{array}{l}\text { The study was } \\
\text { a single block } \\
\text { study with a } \\
35 \% \text { prevalence. } \\
\text { Further, this } \\
\text { study showed } \\
\text { that a past } \\
\text { history of } \\
\text { posterior iliac } \\
\text { bone graft } \\
\text { harvesting had } \\
\text { no significant } \\
\text { value in contrast } \\
\text { to Carragee et al's } \\
\text { (243) proposition } \\
\text { that the site of } \\
\text { the bone graft } \\
\text { is painful in } \\
\text { asymptomatic } \\
\text { patients. }\end{array}$ \\
\hline $\begin{array}{l}\text { Broadhurst } \\
\text { \& Bond } \\
(157)\end{array}$ & $\begin{array}{l}\text { Double-blind } \\
\text { trial of } 40 \\
\text { patients to } \\
\text { determine the } \\
\text { sensitivity and } \\
\text { specificity of } \\
3 \text { commonly } \\
\text { used pain } \\
\text { provocation } \\
\text { tests for } \\
\text { sacroiliac joint } \\
\text { dysfunction } \\
\text { with } \\
\text { suppression of } \\
\text { pain by } 70 \% \\
\text { with injection } \\
\text { of either } \\
\text { normal saline } \\
\text { or lidocaine. }\end{array}$ & $\begin{array}{l}\text { Intraarticular } \\
\text { injection of } \\
4 \mathrm{~mL} \text { of } 1 \% \\
\text { lignocaine or } 4 \\
\mathrm{~mL} \text { of normal } \\
\text { saline into the } \\
\text { painful joint in } \\
20 \text { patients in } \\
\text { each group. }\end{array}$ & $\begin{array}{l}70 \% \text { pain } \\
\text { suppression. }\end{array}$ & 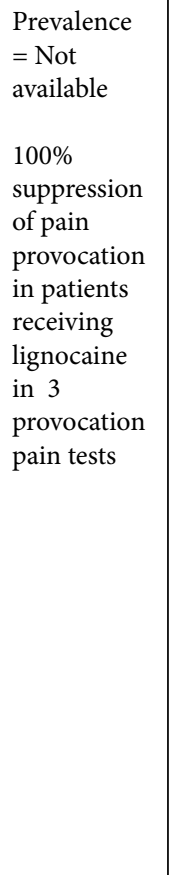 & $\begin{array}{l}\text { Strength: The } \\
\text { study evaluated the } \\
\text { immediate suppression } \\
\text { of pain with } \\
\text { provocative testing } \\
\text { with injection of local } \\
\text { anesthetic or sodium } \\
\text { chloride solution. } \\
\text { Local anesthetic } \\
\text { injection showed 100\% } \\
\text { suppression of pain } \\
\text { provocation tests with } \\
\text { a specificity of } 100 \% \\
\text { for each test and a } \\
\text { sensitivity range of } \\
77 \% \text { to } 87 \% \text {. Weakness: } \\
\text { The study was } \\
\text { performed with high } \\
\text { volumes of solution } \\
\text { which have a tendency } \\
\text { to leak out. Injection of } \\
\text { intraarticular sodium } \\
\text { chloride solution is not } \\
\text { an appropriate model } \\
\text { for placebo testing } \\
\text { (216-223). }\end{array}$ & $11 / 11$ & $\begin{array}{l}\text { The authors } \\
\text { had an excellent } \\
\text { concept of } \\
\text { proving that } \\
\text { placebo is not } \\
\text { effective yielding } \\
100 \% \text { results } \\
\text { with lidocaine } \\
\text { injection, which } \\
\text { was rather } \\
\text { high volumes, } \\
\text { questioning the } \\
\text { accuracy and } \\
\text { validity of the } \\
\text { study. }\end{array}$ \\
\hline
\end{tabular}


Table 9 (cont.). Summary characteristics of studies utilizing $50 \%$ and $70 \%$ relief for single and dual blocks.

\begin{tabular}{|c|c|c|c|c|c|c|c|}
\hline $\begin{array}{c}\text { Reference, } \\
\text { Year }\end{array}$ & $\begin{array}{l}\text { Number of } \\
\text { Patients } \\
\text { Selection } \\
\text { Criteria }\end{array}$ & Intervention & $\begin{array}{l}\text { Outcome } \\
\text { Measures }\end{array}$ & Results & $\begin{array}{c}\text { Strengths } \\
\text { Weaknesses }\end{array}$ & $\begin{array}{l}\text { Methodological } \\
\text { Quality } \\
\text { Assessment } \\
\text { Score } \\
\end{array}$ & Comments \\
\hline \multicolumn{8}{|c|}{ 50\%-79\% RELIEF WITH DUAL BLOCKS } \\
\hline $\begin{array}{l}\text { Maigne et } \\
\text { al (54) }\end{array}$ & $\begin{array}{l}54 \text { patients } \\
\text { aged } 18-75 \\
\text { with chronic } \\
\text { unilateral LBP } \\
\text { with or without } \\
\text { radiation to } \\
\text { the posterior } \\
\text { thigh for }>50 \\
\text { days (median } \\
4.2 \text { months). } \\
\text { Patients had } \\
\text { failed epidural } \\
\text { or lumbar facet } \\
\text { injections. }\end{array}$ & $\begin{array}{l}\text { A screening } \\
\text { block was } \\
\text { done with } 2 \% \\
\text { lidocaine and } \\
\text { a confirmatory } \\
\text { block was } \\
\text { performed } \\
\text { with } \\
\text { bupivacaine } \\
0.5 \% \text {. }\end{array}$ & $\begin{array}{l}\text { Greater than } \\
75 \% \text { relief was } \\
\text { considered a } \\
\text { positive block. }\end{array}$ & $\begin{array}{l}\text { Prevalence }= \\
18.5 \% \\
\text { False- } \\
\text { positive rate } \\
=20 \%\end{array}$ & $\begin{array}{l}\text { Strengths: The study } \\
\text { was performed in a } \\
\text { prospective manner } \\
\text { with dual blocks and } \\
\text { a thorough clinical } \\
\text { examination including } \\
\text { pain provocation } \\
\text { tests. Weaknesses: } \\
\text { The selection criteria } \\
\text { is somewhat rigid } \\
\text { with pain provocation } \\
\text { testing. }\end{array}$ & $8 / 11$ & $\begin{array}{l}\text { The study } \\
\text { questions the } \\
\text { accuracy of some } \\
\text { of the presumed } \\
\text { sacroiliac pain } \\
\text { provocation tests. }\end{array}$ \\
\hline $\begin{array}{l}\text { Irwin et al } \\
\text { (55) }\end{array}$ & $\begin{array}{l}158 \text { patients } \\
\text { underwent } \\
\text { sacroiliac joint } \\
\text { injections } \\
\text { with average } \\
\text { symptoms } \\
\text { duration of } \\
34 \text { months. } \\
\text { Patients failed } \\
\text { conservative } \\
\text { modalities prior } \\
\text { to injection } \\
\text { therapy. }\end{array}$ & $\begin{array}{l}\text { A screening } \\
\text { block with } \\
2 \mathrm{~mL} \text { of } 2 \% \\
\text { lidocaine } \\
\text { for the first } \\
\text { injection, } \\
\text { followed by } 2 \\
\mathrm{~mL} \text { of } 0.25 \% \\
\text { bupivacaine, } \\
\text { a local } \\
\text { anesthetic, } \\
\text { for the } \\
\text { confirmatory } \\
\text { injection. }\end{array}$ & $\begin{array}{l}\text { At least } 70 \% \\
\text { reduction } \\
\text { of familiar } \\
\text { painful } \\
\text { symptoms } \\
\text { after the initial } \\
\text { injection for } \\
3 \text { or } 4 \text { hours } \\
\text { for positive } \\
\text { response. }\end{array}$ & 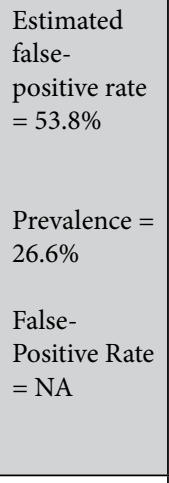 & $\begin{array}{l}\text { Strengths: The } \\
\text { study included a } \\
\text { large proportion of } \\
\text { patients with sacroiliac } \\
\text { joint pain inducing } \\
\text { minimal bias due to a } \\
\text { retrospective nature. } \\
\text { Weaknesses: } \\
\text { A retrospective nature } \\
\text { of the study. }\end{array}$ & $8 / 11$ & $\begin{array}{l}\text { The largest } \\
\text { study to date } \\
\text { utilizing dual } \\
\text { blocks yielding } \\
\text { prevalence of } \\
26.6 \% \text { with an } \\
\text { estimated false- } \\
\text { positive rate of } \\
53.8 \% \text {. }\end{array}$ \\
\hline $\begin{array}{l}\text { DePalma et } \\
\text { al }(78,195)\end{array}$ & $\begin{array}{l}156 \text { patients } \\
\text { underwent } \\
\text { diagnostic } \\
\text { procedures } \\
\text { to evaluate } \\
\text { the source of } \\
\text { chronic low } \\
\text { back pain based } \\
\text { on the age. }\end{array}$ & $\begin{array}{l}\text { Intraarticular } \\
\text { injection of } \\
0.5 \mathrm{~mL} \text { of } \\
\text { anesthetic, } \\
1 \% \text { lidocaine } \\
\text { for first block } \\
\text { with } 0.5 \% \\
\text { bupivacaine } \\
\text { for the second. }\end{array}$ & $\begin{array}{l}\text { At least } 75 \% \\
\text { pain relief for } \\
2 \text { hours for } \\
\text { lidocaine and } \\
8 \text { hours for } \\
\text { bupivacaine. }\end{array}$ & $\begin{array}{l}\text { Prevalence = } \\
18.2 \% \\
\text { False- } \\
\text { Positive } \\
\text { Rate = Not } \\
\text { available }\end{array}$ & $\begin{array}{l}\text { Strengths: The study } \\
\text { was performed in } \\
\text { a heterogenous } \\
\text { population in a } \\
\text { practical setting in a } \\
\text { retrospective manner } \\
\text { overall in a large } \\
\text { proportion of patients. } \\
\text { Weaknesses: } \\
\text { Retrospective } \\
\text { evaluation without } \\
\text { identification of } \\
\text { number of patients } \\
\text { undergoing sacroiliac } \\
\text { joint blocks, thus } \\
\text { not providing actual } \\
\text { prevalence in only } \\
\text { sacroiliac joint } \\
\text { patients. }\end{array}$ & $8 / 11$ & \\
\hline
\end{tabular}


Prevalence and Diagnostic Accuracy of Sacroiliac Joint Interventions

Table 9 (cont.). Summary characteristics of studies utilizing $50 \%$ and $70 \%$ relief for single and dual blocks.

\begin{tabular}{|c|c|c|c|c|c|c|c|}
\hline $\begin{array}{c}\text { Reference, } \\
\text { Year }\end{array}$ & $\begin{array}{l}\text { Number of } \\
\text { Patients } \\
\text { Selection } \\
\text { Criteria } \\
\end{array}$ & Intervention & $\begin{array}{l}\text { Outcome } \\
\text { Measures }\end{array}$ & Results & $\begin{array}{c}\text { Strengths } \\
\text { Weaknesses }\end{array}$ & $\begin{array}{l}\text { Methodological } \\
\text { Quality } \\
\text { Assessment } \\
\text { Score } \\
\end{array}$ & Comments \\
\hline $\begin{array}{l}\text { DePalma } \\
\text { et al (79) }\end{array}$ & $\begin{array}{l}\text { Retrospective } \\
\text { evaluation of } 27 \\
\text { motor vehicle } \\
\text { collision- } \\
\text { induced } \\
\text { chronic low } \\
\text { back pain } \\
\text { patients } \\
\text { undergoing } \\
\text { multiple types } \\
\text { of diagnostic } \\
\text { interventions. }\end{array}$ & $\begin{array}{l}\text { Intraarticular } \\
\text { injection of } \\
0.5 \mathrm{~mL} \text { of } \\
\text { anesthetic, } \\
1 \% \text { lidocaine } \\
\text { for first block } \\
\text { with } 0.5 \% \\
\text { bupivacaine } \\
\text { for the second. }\end{array}$ & $\begin{array}{l}\text { Diagnostic } \\
\text { blockade of } \\
\text { sacroiliac } \\
\text { joints was } \\
\text { deemed } \\
\text { positive if } \\
\text { the patient's } \\
\text { index pain was } \\
\text { relieved by } \\
75 \% \text { or greater } \\
\text { after injection } \\
\text { of each } \\
\text { anesthetic. }\end{array}$ & $\begin{array}{l}\text { Prevalence = } \\
18.2 \% \\
\text { False- } \\
\text { Positive } \\
\text { Rate = Not } \\
\text { available }\end{array}$ & $\begin{array}{l}\text { Strengths: The study } \\
\text { performed only in } \\
\text { patients with motor } \\
\text { vehicle collision with a } \\
\text { retrospective analysis. } \\
\text { Weaknesses: } \\
\text { Retrospective analysis } \\
\text { in a small proportion } \\
\text { of patients involving } \\
\text { multiple etiologies and } \\
\text { with } 7 \text { of } 27 \text { patients or } \\
25.9 \% \text { positive response } \\
\text { rate to dual blocks. } \\
\text { Further, no data is } \\
\text { provided with regards to } \\
\text { the number of patients } \\
\text { undergoing sacroiliac } \\
\text { joint injections. }\end{array}$ & $8 / 11$ & \\
\hline $\begin{array}{l}\text { DePalma } \\
\text { et al (119) }\end{array}$ & $\begin{array}{l}\text { The diagnosis } \\
\text { of } 28 \text { fusion } \\
\text { cases identified } \\
\text { from } 170 \\
\text { low back } \\
\text { pain patients } \\
\text { undergoing } \\
\text { diagnostic } \\
\text { procedures } \\
\text { included } 12 \\
\text { with sacroiliac } \\
\text { joint pain. }\end{array}$ & $\begin{array}{l}\text { Intraarticular } \\
\text { injection of } \\
0.5 \mathrm{~mL} \text { of } \\
\text { anesthetic, } \\
1 \% \text { lidocaine } \\
\text { for first block } \\
\text { with } 0.5 \% \\
\text { bupivacaine } \\
\text { for the second. }\end{array}$ & $\begin{array}{l}\text { Diagnostic } \\
\text { blockade of } \\
\text { sacroiliac joints } \\
\text { was deemed } \\
\text { positive if } \\
\text { the patient's } \\
\text { index pain was } \\
\text { relieved by } 75 \% \\
\text { or greater after } \\
\text { injection of } \\
\text { each anesthetic. }\end{array}$ & $\begin{array}{l}\text { Prevalence = } \\
42.9 \% \\
\text { False- } \\
\text { positive = } \\
\text { NA }\end{array}$ & $\begin{array}{l}\text { The study included } \\
28 \text { fusion cases which } \\
\text { were separated from } \\
\text { others. Weaknesses: } \\
\text { Retrospective } \\
\text { evaluation with rather } \\
\text { small proportion of } \\
\text { patients yielding very } \\
\text { high sacroiliac joint } \\
\text { pain prevalence of } \\
42.9 \% \text { with } 12 \text { out of } \\
28 \text { patients. }\end{array}$ & $8 / 11$ & $\begin{array}{l}\text { A small } \\
\text { retrospective } \\
\text { study yielding a } \\
\text { high percentage } \\
\text { of sacroiliac joint } \\
\text { pain. }\end{array}$ \\
\hline $\begin{array}{l}\text { van der } \\
\text { Wurff et al } \\
(160)\end{array}$ & $\begin{array}{l}\text { Total number } \\
\text { of } 140 \text { patients } \\
\text { with chronic } \\
\text { low back pain } \\
\text { visiting the pain } \\
\text { clinic in the } \\
\text { Netherlands, } 60 \\
\text { patients entered } \\
\text { the study. }\end{array}$ & $\begin{array}{l}\text { The } \\
\text { fluoroscopically } \\
\text { guided contrast } \\
\text { enhanced } \\
\text { sacroiliac joint } \\
\text { injections were } \\
\text { performed } \\
\text { initially with } \\
2 \mathrm{~mL} \text { of } 2 \% \\
\text { lidocaine and } \\
\text { next time } \\
\text { with } 0.25 \% \\
\text { bupivacaine. }\end{array}$ & $\begin{array}{l}\text { A reduction } \\
\text { in the patient's } \\
\text { characteristic } \\
\text { pain of } 50 \% \text { or } \\
\text { more on the } \\
\text { VAS remaining } \\
\text { for at least } \\
\text { one hour for } \\
\text { lidocaine or } \\
4 \text { hours for } \\
\text { bupivacaine } \\
\text { was considered } \\
\text { as positive. } \\
\text { When a patient } \\
\text { showed a VAS } \\
\text { reduction } \\
\text { after both } \\
\text { intraarticular } \\
\text { sacroiliac joint } \\
\text { blocks, this } \\
\text { was considered } \\
\text { a positive } \\
\text { response. Any } \\
\text { other outcome } \\
\text { was considered } \\
\text { a negative } \\
\text { response. }\end{array}$ & $\begin{array}{l}\text { Prevalence = } \\
38 \% \\
\text { False- } \\
\text { positive rate } \\
=21 \%\end{array}$ & $\begin{array}{l}\text { Strengths: The study is } \\
\text { performed in a group } \\
\text { of patients drawn from } \\
\text { a large proportion with } \\
\text { a large proportion of } \\
\text { patients in sacroiliac } \\
\text { joint pain group itself. } \\
\text { Weaknesses: There was } \\
\text { leakage of the fluids in } \\
5 \text { of } 60 \text { patients with } \\
\text { sciatic nerve palsy. } 60 \\
\text { patients of } 140 \text { with } \\
\text { suspected sacroiliac } \\
\text { joint pain appears } \\
\text { to be the Center is } \\
\text { more geared towards } \\
\text { sacroiliac joint pain. } \\
\text { The second weakness } \\
\text { is of the } 50 \% \text { or greater } \\
\text { pain relief rather than } \\
\text { a criterion standard of } \\
80 \% \text { or greater. }\end{array}$ & $9 / 11$ & $\begin{array}{l}\text { Well performed } \\
\text { study in a large } \\
\text { proportion of } \\
\text { patients with a } \\
\text { weakness of } 50 \% \\
\text { pain relief, thus } \\
\text { maybe resulting } \\
\text { in higher } \\
\text { prevalence rate } \\
\text { of } 38 \% \text {. }\end{array}$ \\
\hline
\end{tabular}


Pain Physician: May/June 2012; 15:E305-E344

Table 9 (cont.). Summary characteristics of studies utilizing $50 \%$ and $70 \%$ relief for single and dual blocks.

\begin{tabular}{|c|c|c|c|c|c|c|c|}
\hline $\begin{array}{c}\text { Reference, } \\
\text { Year }\end{array}$ & $\begin{array}{l}\text { Number of } \\
\text { Patients } \\
\text { Selection } \\
\text { Criteria }\end{array}$ & Intervention & $\begin{array}{l}\text { Outcome } \\
\text { Measures }\end{array}$ & Results & $\begin{array}{c}\text { Strengths } \\
\text { Weaknesses }\end{array}$ & $\begin{array}{l}\text { Methodological } \\
\text { Quality } \\
\text { Assessment } \\
\text { Score }\end{array}$ & Comments \\
\hline $\begin{array}{l}\text { Liliang et } \\
\text { al (194) }\end{array}$ & $\begin{array}{l}52 \text { patients } \\
\text { were selected } \\
\text { from } 130 \\
\text { patients after } \\
\text { undergoing } \\
\text { lumbar or } \\
\text { lumbosacral } \\
\text { fusions who } \\
\text { met the criteria } \\
\text { for at least } 3 \text { of } \\
\text { the provocative } \\
\text { tests for } \\
\text { sacroiliac joint } \\
\text { pain. }\end{array}$ & $\begin{array}{l}\text { Intraarticular } \\
\text { injection } \\
\text { with either } \\
\text { lidocaine ( } 2 \%) \\
\text { or bupivacaine } \\
(0.5 \%), 1 \mathrm{~mL} \text {, } \\
\text { mixed with } \\
40 \mathrm{mg} \text { of } \\
\text { triamcinolone. }\end{array}$ & $\begin{array}{l}75 \% \text { pain } \\
\text { relief for } 1 \\
\text { to } 4 \text { hours } \\
\text { following the } \\
\text { sacroiliac joint } \\
\text { blocks. }\end{array}$ & $\begin{array}{l}\text { Prevalence = } \\
40 \% \\
\text { False- } \\
\text { positive rate } \\
=26 \%\end{array}$ & $\begin{array}{l}\text { Strengths The study } \\
\text { is one of the more } \\
\text { recent and well } \\
\text { performed large } \\
\text { studies, specifically in } \\
\text { sacroiliac joint fusion. } \\
\text { Weaknesses: } \\
\text { Only } 52 \text { patients and } \\
\text { utilized } 75 \% \text { pain } \\
\text { relief. }\end{array}$ & $8 / 11$ & $\begin{array}{l}\text { With } 75 \% \\
\text { pain relief, the } \\
\text { results appear } \\
\text { to be highly } \\
\text { appropriate with } \\
\text { highly selected } \\
\text { population. }\end{array}$ \\
\hline \multicolumn{8}{|c|}{$80 \%$ TO $100 \%$ RELIEF WITH A SINGLE BLOCK } \\
\hline $\begin{array}{l}\text { Pang et al } \\
(42)\end{array}$ & $\begin{array}{l}\text { In this } \\
\text { prospective } \\
\text { evaluation, } 104 \\
\text { consecutive adult } \\
\text { patients who } \\
\text { underwent spinal } \\
\text { pain mapping } \\
\text { were examined } \\
\text { and analyzed. } \\
\text { They found in } \\
\text { this group a total } \\
\text { of } 87 \% \text { of the } \\
\text { patients with } \\
\text { diagnosed pain } \\
\text { source and 13\% } \\
\text { without a source. } \\
\text { In this evaluation, } \\
\text { sacroiliac } \\
\text { joint pain was } \\
\text { identified in } 10 \% \\
\text { of the patients } \\
\text { from the total } \\
\text { sample. }\end{array}$ & $\begin{array}{l}\text { Intraarticular } \\
\text { injection with } \\
2 \mathrm{~mL} \text { of } 2 \% \\
\text { lidocaine }\end{array}$ & $90 \%$ pain relief & $\begin{array}{l}\text { Prevalence }= \\
10 \% \text { of total } \\
\text { sample. }\end{array}$ & $\begin{array}{l}\text { Strengths: This study } \\
\text { was performed in a } \\
\text { large proportion of } \\
\text { patients for spinal } \\
\text { mapping purposes } \\
\text { identifying various } \\
\text { causes. Weaknesses: } \\
\text { The study does not } \\
\text { provide detailed data } \\
\text { on sacroiliac joint pain. }\end{array}$ & $8 / 11$ & $\begin{array}{l}\text { Even though } \\
\text { this is a well } \\
\text { performed } \\
\text { study in a large } \\
\text { proportion } \\
\text { of patients, it } \\
\text { is not known } \\
\text { the number of } \\
\text { patients included } \\
\text { for sacroiliac } \\
\text { joint pain, } \\
\text { thus we do not } \\
\text { know the true } \\
\text { prevalence of } \\
\text { sacroiliac joint } \\
\text { pain even with a } \\
\text { single block. }\end{array}$ \\
\hline $\begin{array}{l}\text { Dreyfuss et } \\
\text { al (148) }\end{array}$ & $\begin{array}{l}\text { The prospective } \\
\text { study included } \\
85 \text { patients } \\
\text { based on } \\
\text { historical data } \\
\text { with } 12 \text { tests } \\
\text { performed by } 2 \\
\text { examiners. }\end{array}$ & $\begin{array}{l}\text { Intraarticular } \\
\text { injection of } \\
1.5 \mathrm{~mL} \text { of } 2 \% \\
\text { lignocaine } \\
\text { and } 0.5 \mathrm{~mL} \\
\text { of Celestone } \\
\text { Soluspan } \\
\text { unless a firm } \\
\text { endpoint was } \\
\text { reached before } \\
\text { this volume. }\end{array}$ & $\begin{array}{l}90 \% \text { or more } \\
\text { relief was } \\
\text { considered } \\
\text { a positive } \\
\text { response, and } \\
\text { less than } 90 \% \\
\text { relief was } \\
\text { considered } \\
\text { a negative } \\
\text { response. }\end{array}$ & $\begin{array}{l}\text { Prevalence }= \\
45 \text { out of } 85 \\
(53 \%)\end{array}$ & $\begin{array}{l}\text { Strengths: The study } \\
\text { was performed } \\
\text { collaboratively with } \\
\text { extremely careful } \\
\text { patient selection } \\
\text { undergoing } 12 \text { clinical } \\
\text { tests, which included } \\
\text { multiple provocative } \\
\text { maneuvers. } \\
\text { Weaknesses: } 5 \text { patients } \\
\text { were excluded from } \\
\text { the analysis. Single } \\
\text { block with a specific } \\
\text { selection criteria. }\end{array}$ & $8 / 11$ & $\begin{array}{l}\text { The results } \\
\text { showed fairly } \\
\text { high proportion } \\
\text { of patients with } \\
\text { sacroiliac joint } \\
\text { pain due to } \\
\text { strict selection } \\
\text { criteria. However, } \\
\text { there were } \\
\text { no historical } \\
\text { features, with } \\
\text { none of the } 12 \\
\text { sacroiliac joint } \\
\text { tests and any } \\
\text { combination of } \\
\text { these } 12 \text { tests } \\
\text { demonstrating } \\
\text { worthwhile } \\
\text { diagnostic value. }\end{array}$ \\
\hline
\end{tabular}


Prevalence and Diagnostic Accuracy of Sacroiliac Joint Interventions

Table 9 (cont.). Summary characteristics of studies utilizing $50 \%$ and $70 \%$ relief for single and dual blocks.

\begin{tabular}{|c|c|c|c|c|c|c|c|}
\hline $\begin{array}{c}\text { Reference, } \\
\text { Year }\end{array}$ & $\begin{array}{l}\text { Number of } \\
\text { Patients } \\
\text { Selection } \\
\text { Criteria }\end{array}$ & Intervention & $\begin{array}{l}\text { Outcome } \\
\text { Measures }\end{array}$ & Results & $\begin{array}{c}\text { Strengths } \\
\text { Weaknesses }\end{array}$ & $\begin{array}{l}\text { Methodological } \\
\text { Quality } \\
\text { Assessment } \\
\text { Score }\end{array}$ & Comments \\
\hline $\begin{array}{l}\text { Slipman et } \\
\text { al (152) }\end{array}$ & $\begin{array}{l}50 \text { consecutive } \\
\text { patients } \\
\text { meeting a } \\
\text { pre-established } \\
\text { criteria from a } \\
\text { chronic spine } \\
\text { practice. }\end{array}$ & $\begin{array}{l}\text { Intraarticular } \\
\text { injection } \\
\text { of } 1 \mathrm{~mL} \text { of } \\
\text { betamethasone } \\
\text { sodium } \\
\text { phosphate } \\
\text { and acetate } \\
\text { suspension, } \\
60 \mathrm{mg} \text { per } \\
\mathrm{mL}, 3 \mathrm{~mL} \text { of } \\
1 \% \text { lidocaine } \\
\text { hydrochloride, } \\
\text { or } 3 \mathrm{~mL} \text { of } \\
2 \% \text { lidocaine } \\
\text { hydrochloride. } \\
\text { Among the } \\
\text { patients } \\
\text { with positive } \\
\text { response, } \\
\text { there were } 27 \\
\text { patients with } \\
\text { negative scans } \\
\text { and } 4 \text { patients } \\
\text { with positive } \\
\text { scans. }\end{array}$ & $\begin{array}{l}\text { A reduction } \\
\text { of the VAS } \\
\text { rating by at } \\
\text { least } 80 \% \text { was } \\
\text { considered } \\
\text { a positive } \\
\text { response to } \\
\text { sacroiliac joint } \\
\text { block. }\end{array}$ & $\begin{array}{l}\text { Prevalence }= \\
62 \%\end{array}$ & $\begin{array}{l}\text { Strengths: The study } \\
\text { was performed to } \\
\text { evaluate the value of } \\
\text { radionuclide imaging } \\
\text { in the diagnosis } \\
\text { of sacroiliac joint } \\
\text { syndrome in patients } \\
\text { who were positive } \\
\text { for at least } 3 \text { positive } \\
\text { responses to include } \\
2 \text { specific stress } \\
\text { maneuvers. All the } \\
\text { patients underwent } \\
\text { radionuclide imaging. } \\
\text { Weaknesses: } \\
\text { Stringent selection } \\
\text { criteria yielding high } \\
\text { prevalence of sacroiliac } \\
\text { joint pain with a single } \\
\text { block. }\end{array}$ & $8 / 11$ & $\begin{array}{l}\text { This study shows } \\
\text { low sensitivity } \\
\text { and high } \\
\text { specificity of } \\
\text { nuclear imaging } \\
\text { in the evaluation } \\
\text { of sacroiliac joint } \\
\text { syndrome. }\end{array}$ \\
\hline $\begin{array}{l}\text { Laslett et al } \\
\text { (154) }\end{array}$ & $\begin{array}{l}\text { Prospective } \\
\text { evaluation of } \\
48 \text { patients } \\
\text { satisfying } \\
\text { inclusion } \\
\text { criteria from } \\
\text { a total of } 62 \\
\text { patients agreed } \\
\text { to participate } \\
\text { and were } \\
\text { evaluated. } \\
\text { Patients with } \\
\text { buttock pain, } \\
\text { with or without } \\
\text { lumbar or } \\
\text { lower extremity } \\
\text { symptoms were } \\
\text { included. }\end{array}$ & $\begin{array}{l}\text { Intraarticular } \\
\text { injection of } \\
1 \mathrm{~mL} \text { of } 2 \% \\
\text { lignocaine. } \\
\text { All patients } \\
\text { underwent } \\
\text { provocation } \\
\text { testing. }\end{array}$ & $\begin{array}{l}\text { At least } 80 \% \\
\text { pain relief }\end{array}$ & $\begin{array}{l}\text { Prevalence } \\
=16 \text { of } 48 \\
(33 \%)\end{array}$ & $\begin{array}{l}\text { Strengths: All patients } \\
\text { underwent provocation } \\
\text { testing. Authors } \\
\text { essentially were } \\
\text { comparing validity of } \\
\text { individual provocation } \\
\text { tests and composites } \\
\text { of tests. They reported } \\
\text { sensitivity and } \\
\text { specificity for } 3 \text { or } \\
\text { more of } 6 \text { positive } \\
\text { sacroiliac tests } \\
\text { were } 94 \% \text { and } 78 \% \\
\text { respectively. Well } \\
\text { performed study } \\
\text { with evaluation of } \\
\text { validity of individual } \\
\text { provocation tests and } \\
\text { composites of tests. } \\
\text { Weaknesses: Strict } \\
\text { selection criteria } \\
\text { probably yielding } \\
\text { higher prevalence. }\end{array}$ & $8 / 11$ & $\begin{array}{l}\text { The authors } \\
\text { concluded that } \\
\text { composites of } \\
\text { provocation } \\
\text { sacroiliac joint } \\
\text { tests are of } \\
\text { value in clinical } \\
\text { diagnosis of } \\
\text { symptomatic } \\
\text { sacroiliac joint } \\
\text { pain when } 3 \\
\text { or more of the } \\
6 \text { tests were } \\
\text { positive, with } \\
\text { the greatest } \\
\text { applicability } \\
\text { when } 4 \text { tests } \\
\text { were positive. } \\
\text { When none of } \\
\text { the provocation } \\
\text { tests provoked } \\
\text { familiar pain, the } \\
\text { sacroiliac joint } \\
\text { can be ruled out } \\
\text { as a source of } \\
\text { current low back } \\
\text { pain. }\end{array}$ \\
\hline
\end{tabular}


Pain Physician: May/June 2012; 15:E305-E344

Table 9 (cont.). Summary characteristics of studies utilizing $50 \%$ and $70 \%$ relief for single and dual blocks.

\begin{tabular}{|c|c|c|c|c|c|c|c|}
\hline $\begin{array}{c}\text { Reference, } \\
\text { Year }\end{array}$ & $\begin{array}{l}\text { Number of } \\
\text { Patients } \\
\text { Selection } \\
\text { Criteria } \\
\end{array}$ & Intervention & $\begin{array}{l}\text { Outcome } \\
\text { Measures }\end{array}$ & Results & $\begin{array}{c}\text { Strengths } \\
\text { Weaknesses }\end{array}$ & $\begin{array}{l}\text { Methodological } \\
\text { Quality } \\
\text { Assessment } \\
\text { Score } \\
\end{array}$ & Comments \\
\hline $\begin{array}{l}\text { Young et al } \\
\text { (155) }\end{array}$ & $\begin{array}{l}\text { A prospective } \\
\text { evaluation of } \\
81 \text { patients } \\
\text { with chronic } \\
\text { lumbopelvic } \\
\text { pain to evaluate } \\
\text { correlation } \\
\text { of clinical } \\
\text { examination } \\
\text { characteristics } \\
\text { with } 3 \text { sources } \\
\text { of chronic low } \\
\text { back pain with } \\
\text { diagnostic } \\
\text { injections } \\
\text { as criterion } \\
\text { standard. } 57 \\
\text { patients were } \\
\text { suspected with } \\
\text { sacroiliac joint } \\
\text { pain. }\end{array}$ & $\begin{array}{l}\text { Intraarticular } \\
\text { injection with } \\
1.5 \mathrm{~mL} \text { of } \\
\text { lidocaine }\end{array}$ & $\begin{array}{l}\text { At least } 80 \% \\
\text { pain relief }\end{array}$ & $\begin{array}{l}\text { Prevalence }= \\
39 \%\end{array}$ & $\begin{array}{l}\text { Strengths: The authors } \\
\text { evaluated correlation } \\
\text { of clinical examination } \\
\text { characteristics with } 3 \\
\text { sources of chronic low } \\
\text { back pain including } \\
\text { sacroiliac joint pain } \\
\text { simulating practical } \\
\text { setting. They found } \\
\text { strongest relationships } \\
\text { were seen between } \\
\text { sacroiliac joint pain } \\
\text { and } 3 \text { or more pain } \\
\text { provocation tests. } \\
\text { Weaknesses: Highly } \\
\text { selective group of } \\
\text { patients with positive } \\
\text { provocation tests }\end{array}$ & $8 / 11$ & $\begin{array}{l}\text { The authors } \\
\text { illustrate } \\
\text { the positive } \\
\text { correlation } \\
\text { with strongest } \\
\text { relationships } \\
\text { between } \\
\text { sacroiliac joint } \\
\text { pain and } 3 \text { or } \\
\text { more positive } \\
\text { pain provocation } \\
\text { tests. }\end{array}$ \\
\hline $\begin{array}{l}\text { Stanford \& } \\
\text { Burnham } \\
(175)\end{array}$ & $\begin{array}{l}\text { Evaluation of } \\
34 \text { patients } \\
\text { with suspected } \\
\text { unilateral } \\
\text { mechanical } \\
\text { sacroiliac joint } \\
\text { pain. }\end{array}$ & $\begin{array}{l}\text { Intraarticular } \\
\text { injection of } \\
1.5 \mathrm{~mL} \text { of } \\
2 \% \text { lidocaine } \\
\text { and } 1 \mathrm{~mL} \text { of } \\
\text { corticosteroid. }\end{array}$ & $\begin{array}{l}\text { A positive } \\
\text { block was } \\
\text { defined as } \\
\text { greater than } \\
79 \% \text { index } \\
\text { pain relief } \\
\text { within the } \\
\text { first } 2 \text { hours } \\
\text { post-injection. }\end{array}$ & $\begin{array}{l}\text { Prevalence }= \\
32 \%\end{array}$ & $\begin{array}{l}\text { Strengths: The study } \\
\text { was performed in a } \\
\text { practical setting to } \\
\text { evaluate usefulness to } \\
\text { repeat sacroiliac joint } \\
\text { provocative testing post } \\
\text { block. Weaknesses: The } \\
\text { small number of patients } \\
\text { with a single block and } \\
\text { strict selection criteria. }\end{array}$ & $6 / 11$ & $\begin{array}{l}\text { The study } \\
\text { illustrates utility } \\
\text { of pre-block } \\
\text { sacroiliac joint } \\
\text { provocative tests. }\end{array}$ \\
\hline \multicolumn{8}{|c|}{ 80\% TO 100\% RELIEF WITH DUAL BLOCKS } \\
\hline $\begin{array}{l}\text { Manchikanti } \\
\text { et al (41) }\end{array}$ & $\begin{array}{l}120 \text { patients } \\
\text { (age 18-90) } \\
\text { presenting to } \\
\text { the clinic with } \\
>6 \text { months } \\
\text { of low back } \\
\text { pain and no } \\
\text { structural basis } \\
\text { for the pain by } \\
\text { radiographic } \\
\text { imaging. } 20 \\
\text { patients were } \\
\text { evaluated for SI } \\
\text { joint pain. }\end{array}$ & $\begin{array}{l}\text { The screening } \\
\text { block was } \\
\text { done with } \\
2 \% \text { lidocaine } \\
\text { and the } \\
\text { confirmatory } \\
\text { block was } \\
\text { performed } \\
\text { using } 0.5 \% \\
\text { bupivacaine. }\end{array}$ & $\begin{array}{l}\text { At least } 80 \% \\
\text { pain relief } \\
\text { with ability } \\
\text { to perform } \\
\text { previously } \\
\text { painful } \\
\text { movements } \\
\text { with } \\
\text { concordant } \\
\text { relief based } \\
\text { on the local } \\
\text { anesthetic } \\
\text { injected. }\end{array}$ & $\begin{array}{l}\text { Prevalence } \\
10 \% \\
\text { False- } \\
\text { Positive Rate } \\
22 \%\end{array}$ & $\begin{array}{l}\text { Strengths: The study was } \\
\text { performed to evaluate } \\
\text { relative contributions } \\
\text { of various structures } \\
\text { in chronic low back } \\
\text { pain in } 120 \text { patients, } \\
\text { even though only } 20 \\
\text { patients were evaluated } \\
\text { for sacroiliac joint pain. } \\
\text { Dual blocks were utilized } \\
\text { with } 80 \% \text { pain relief as } \\
\text { the criterion standard. } \\
\text { Weaknesses: Of the } \\
120 \text { patients, only } 20 \\
\text { patients were suspected } \\
\text { of sacroiliac joint pain } \\
\text { or underwent sacroiliac } \\
\text { joint blocks. }\end{array}$ & $9 / 11$ & $\begin{array}{l}\text { The study } \\
\text { illustrates a low } \\
\text { proportion of } \\
\text { sacroiliac joint } \\
\text { pain in } 10 \% \\
\text { of the patients } \\
\text { with suspected } \\
\text { sacroiliac joint } \\
\text { pain. }\end{array}$ \\
\hline
\end{tabular}


Prevalence and Diagnostic Accuracy of Sacroiliac Joint Interventions

Table 9 (cont.). Summary characteristics of studies utilizing $50 \%$ and $70 \%$ relief for single and dual blocks.

\begin{tabular}{|c|c|c|c|c|c|c|c|}
\hline $\begin{array}{c}\text { Reference, } \\
\text { Year }\end{array}$ & $\begin{array}{l}\text { Number of } \\
\text { Patients } \\
\text { Selection } \\
\text { Criteria }\end{array}$ & Intervention & $\begin{array}{l}\text { Outcome } \\
\text { Measures }\end{array}$ & Results & $\begin{array}{c}\text { Strengths } \\
\text { Weaknesses }\end{array}$ & $\begin{array}{l}\text { Methodological } \\
\text { Quality } \\
\text { Assessment } \\
\text { Score }\end{array}$ & Comments \\
\hline $\begin{array}{l}\text { Laslett et al } \\
\text { (159) }\end{array}$ & $\begin{array}{l}48 \text { patients } \\
\text { received initial } \\
\text { sacroiliac joint } \\
\text { diagnostic } \\
\text { injection, } \\
\text { derived from } \\
62 \text { patients with } \\
\text { buttock pain } \\
\text { with or without } \\
\text { lumbar or } \\
\text { lower extremity } \\
\text { symptoms. }\end{array}$ & $\begin{array}{l}\text { Intraarticular } \\
\text { injection of } \\
\text { less than } 1.5 \\
\mathrm{~mL} \text { of local } \\
\text { anesthetic } \\
\text { lidocaine for } \\
\text { initial block } \\
\text { followed by } \\
\text { bupivacaine } \\
\text { for the } \\
\text { confirmatory } \\
\text { block. }\end{array}$ & $\begin{array}{l}\text { At least } 80 \% \\
\text { reduction in } \\
\text { pain for the } \\
\text { duration of } \\
\text { anesthetic } \\
\text { effect. }\end{array}$ & $\begin{array}{l}\text { Prevalence } \\
=25.6 \%\end{array}$ & $\begin{array}{l}\text { Strengths: Provocation } \\
\text { sacroiliac tests were used } \\
\text { to identify sacroiliac joint } \\
\text { pain as part of the clinical } \\
\text { reasoning process. The } \\
\text { study also illustrated the } \\
\text { diagnostic accuracy of } \\
\text { the clinical examination } \\
\text { and clinical reasoning } \\
\text { process was superior to } \\
\text { the sacroiliac joint pain } \\
\text { provocation tests alone. } \\
\text { Weaknesses: This is a } \\
\text { validity study of sacroiliac } \\
\text { provocation tests. }\end{array}$ & $8 / 11$ & $\begin{array}{l}\text { The authors show } \\
\text { the prevalence } \\
\text { of } 45.6 \% \text { in a } \\
\text { select group of } \\
\text { patients with } \\
\text { clinical reasoning } \\
\text { in addition to } \\
\text { provocation } \\
\text { testing being } \\
\text { superior to } \\
\text { provocation } \\
\text { testing alone. }\end{array}$ \\
\hline
\end{tabular}

showed a prevalence rate of between $30 \%$ and $35 \%$ $(82,83)$.

\subsubsection{Dual Blocks with $\mathbf{5 0 \%}$ to $\mathbf{8 0} \%$ Pain Relief}

There were 7 studies evaluating $50 \%$ to $80 \%$ relief with dual blocks $(54,55,78,79,119,160,194,195)$ with one duplicate study $(78,195)$.

As one might expect, the prevalence rate was lower in the $50 \%$ to $80 \%$ dual block category, especially when $75 \%$ pain relief was utilized as the criterion standard with approximate prevalence of $18 \%$ $(54,78,79,119,195)$. It was higher $(40.4 \%)$ in one study in highly selected population (194). However, when $50 \%$ relief with dual blocks was utilized as the criterion standard, the prevalence rate was shown to be $38 \%$ with a false-positive rate of $21 \%$ (160). Increasing the threshold to $75 \%$ does not appear to reduce the accuracy. Irwin et al (55), in a large retrospective evaluation, found a prevalence rate of $26.6 \%$ using $70 \%$ pain relief. These findings suggest that increasing the cutoff threshold results in lower estimated prevalence rates.

\subsubsection{Single Block with $80 \%$ to $100 \%$ Relief}

There were a total of 6 studies meeting the inclusion criteria evaluating sacroiliac joint pain using a cutoff threshold between $80 \%$ and $100 \%$ relief following a single block $(42,148,152,154,155,175)$.

The prevalence in this group ranged from a low of $10 \%$ to a high of $62 \%$. The $53 \%$ and $62 \%$ prevalence rates reported by Dreyfuss et al (148) and Slipman et al
(152), respectively, were found in highly selected populations. Dreyfuss et al (148) employed a reference standard of greater than $90 \%$ pain relief during the blocks, and enrolled study patients who had pain predominantly below L5. Slipman et al (152) used $80 \%$ pain relief as the criterion standard, and studied a population who had a positive response to 3 sacroiliac joint pain provocation tests. Overall, a single block using $80 \%$ to $100 \%$ pain relief as the reference standard appears to yield prevalence of around $35 \%$.

\subsubsection{Dual Blocks with $80 \%$ to $100 \%$ Relief}

There were a total of 2 studies meeting the inclusion criteria $(41,159)$.

Using between $80 \%$ and $100 \%$ pain relief with dual blocks as the criterion standard has been advocated by some as the most rigorous means for diagnosing sacroiliac joint pain $(2,35,38,39,88)$. In a small study that included only 20 patients, Manchikanti et al (41) found a low prevalence rate of $10 \%$. In contrast, Laslett et al (159) showed a prevalence rate of $25.6 \%$ in a study involving 48 subjects. False-positive rate was $22 \%$ (41). Laslett et al have not estimated the falsepositive rate, but looking at the data, it appears to be $0 \%$ (159).

\subsection{Level of Evidence}

Based on the USPSTF criteria, the evidence was classified to be either good, fair, or limited (or poor). 


\subsubsection{Single Block with $50 \%$ to $80 \%$ Relief}

Based on 3 high quality studies $(82,83,157)$, the evidence is fair.

\subsubsection{Dual Blocks with $50 \%$ to $80 \%$ Relief}

Based on 7 high quality studies $(54,55,78$, $79,119,160,194,195)$ containing over 500 patients, the evidence in this group was good, especially when greater than $70 \%$ pain relief was utilized as the criterion standard $(n=5)$.

\subsubsection{Single Block with $80 \%$ to $100 \%$ Relief}

The evidence is good based on the results of 6 studies $(42,148,152,154,155,175)$, with 5 of them rated as high quality $(42,148,152,154,155)$.

\subsubsection{Dual Blocks with $80 \%$ to $100 \%$ Relief}

The evidence is good based on 2 high-quality studies $(41,159)$ using dual blocks.

\subsubsection{Summary of Evidence}

Overall, there was no significant difference when $70 \%$ or greater relief is utilized as the criterion standard with dual blocks, with good evidence based on multiple high quality studies.

\subsection{Pain Patterns}

Sacroiliac joint pain patterns, referral zones, and intensity mapping have all been evaluated $(148,153,158,161-165)$. In 1994, Fortin et al (162) generated pain referral maps based on provocative injections performed in asymptomatic volunteers, and pain diagrams drawn by patients with low back pain secondary to sacroiliac joint pathology or other sources (i.e., facetogenic and discogenic pain). The authors found that individuals whose point of maximum discomfort fell within an area that extended $10 \mathrm{~cm}$ caudal and $3 \mathrm{~cm}$ lateral to the posterior superior iliac spine were more likely have sacroiliac joint pain $(162,163)$. This finding is supported by the work of Murakami et al (187), who found that patients who responded to low-volume periarticular sacroiliac joint injections could pinpoint their pain to within $2 \mathrm{~cm}$ of the posterior superior iliac spine. In a cross-sectional study by Schwarzer et al (82), the authors found groin pain to be the best means to distinguish sacroiliac joint pain from other causes of back pain. In contrast, Dreyfuss et al (148) found that groin pain was not a discriminating feature of sacroiliac joint pain.

Slipman et al (153) performed an analytical study that sought to identify sacroiliac joint referral patterns in 50 patients who obtained $>80 \%$ pain relief after a single intraarticular injection. The most frequent referral zones were the buttocks (94\%) and lower lumbar region. Fifty percent of patients experienced extension into the lower extremity, $28 \%$ reported pain in the lower leg, and $14 \%$ described groin pain. A study by van der Wurff et al (161) compared pain referral patterns in patients with sacroiliac joint pain and those with other sources of back pain, based on response to dual comparative local anesthetic blocks. They concluded that pain diagrams could not reliably distinguish between low back pain patients suffering from sacroiliac joint pain and those with other primary pain generators. Finally, Jung et al (164) evaluated the usefulness of pain distribution pattern assessment in decision-making in 419 patients with either lumbar facet joint pain or sacroiliac joint pain. The authors found several different patterns for sacroiliac joint pain, such as buttock pain, buttock pain extending into the posterolateral thigh, and buttock pain radiating into the groin. They concluded that pain diagrams could be useful in predicting outcomes from injections and radiofrequency neurotomy.

In summary, although pain patterns may be helpful in identifying patients who might benefit from diagnostic injections, they are not pathognomonic.

\subsection{Necessity for Fluoroscopically Guided Injections}

Sacroiliac joint injections have been performed by many means including without imaging guidance based on clinical examination and tenderness (183). However, the present gold standard is to perform them under fluoroscopy using controlled diagnostic blocks for diagnostic purposes. In an observational study performed in 60 patients, Hansen (191) found that blind injections done by a single practitioner with $2 \mathrm{ml}$ of contrast resulted in intraarticular spread in only $12 \%$ of patients.

In a double-blind study, Rosenberg et al (192) evaluated the accuracy of clinically guided sacroiliac joint injections using computerized tomography. Among the 39 injections performed in 33 patients, intraarticular injection was accomplished in only $22 \%$ of the patients, though the injected contrast was noted to be within one $\mathrm{cm}$ of the joint in $68 \%$ of individuals. Furthermore, injected material was found to be within the sacral foramina in $44 \%$ of cases- usually at S1- and in the epidural space following $24 \%$ of injections. The authors concluded that the low rate of intraarticular injection observed with landmark-guided techniques warranted the use of image guidance in those 
at high risk for failure and complications.

Although expensive and not feasible in routine practice, MRI-guided corticosteroid injections of the sacroiliac joints have been reported to be effective $(171,179,186)$.

Multiple authors have described the feasibility of ultrasound-guided sacroiliac joint injections with consideration of anatomic landmarks $(166,169,176,178,183,185,190)$, but no systematic evaluation has been performed. Further, Hartung et al (166) performed a small study in which ultrasound was used to guide 20 sacroiliac joint injections in 14 patients with sacroiliitis. MRI verification revealed that only $40 \%$ of injections were intraarticular, with the rest being outside the joint cavity. No differences in outcomes were noted between the intra- and extraarticular groups. Unguided sacroiliac joint injections also have been shown to be effective in some studies (183).

In summary, there is no evidence to support the use of ultrasound or landmark-guided injections for sacroiliac joint pain. These injections must be performed under fluoroscopic or radiologic guidance.

\subsection{Accuracy of Testing of Provocation Maneuvers}

Provocation testing has been extensively employed in an attempt for diagnosing sacroiliac joint pain. Multiple studies have also been performed to evaluate the validity of clinical examination, which incorporates provocation testing. The recommended reference standard involves either anesthetic or provocative injections; however, doubts have even been cast on the validity of a sacroiliac joint block as a diagnostic gold standard. A review by Berthelot et al (89) concluded clinical signs and maneuvers to be unreliable for diagnosing pain originating within the sacroiliac joint; they are fraught with both low sensitivity and specificity. But the authors also concluded that sacroiliac joint blocks were similarly unreliable, since pain patterns formerly attributed to the sacroiliac joint can be related to extraarticular structures, most notably the numerous ligaments surrounding the joint. However, multiple studies evaluated in the same studies have reached different conclusions.

In 2 separate systematic reviews, Szadek et al (80) and Hancock et al (49) showed a positive correlation between a battery of provocation testing and diagnostic blocks. Hancock et al (49) included 6 studies in their analysis, while Szadek et al (80) included 15 studies. In a systematic appraisal of the literature assessing the ac- curacy of multiple tests for back pain utilizing QUADAS criteria, Simpson and Gemmell (200) identified 5 studies that focused on sacroiliac joint pain. They found no single test to be consistently valid.

Among the multiple studies utilized to evaluate provocative testing and clinical evaluation $(54,82,148$, $154,155,157,159,160,175,189,200,201)$, different conclusions were reached. All studies were performed utilizing fluoroscopically guided intraarticular injections as the reference standard $(54,82,148,154,155,157,159$, $160,175,189)$. Schwarzer et al $(82)$ found that none of the conventional sacroiliac joint examination procedures tested could reliably discriminate patients with sacroiliac joint pain from those with other sources of back pain. Dreyfuss et al (148) evaluated the value of medical history and physical examination in diagnosing sacroiliac joint pain by assessing 12 overall tests, along with pain diagrams. These provocation tests included the Gillet test, thigh thrust, Patrick test, Gaenslen test, and midline sacral thrust. The results refuted the notion that any single historical or exam finding could reliably identify a painful sacroiliac joint.

Laslett et al and colleagues $(154,155,159,189)$ published multiple studies on the validity of pain provocation testing in the identification of sacroiliac joint dysfunction. In one study, Laslett et al (154) evaluated the validity of provocation tests individually and collectively to predict response to a single diagnostic sacroiliac joint injection. All patients with a positive response to a diagnostic injection reported pain during at least one sacroiliac joint test. The sensitivity and specificity for 3 or more of the 6 tests were $94 \%$ and $78 \%$ respectively. The greatest area under the curve of any of the 2 best 4 tests was 0.842 . The authors concluded that composites of provocation sacroiliac joint tests were of value in the clinical diagnosis of symptomatic sacroiliac joint pain and 3 or more out of 6 tests or have the best predictive power in relation to the results of intraarticular anesthetic blocks. The most valid battery of maneuvers were the distraction, compression, and thigh and sacral thrust tests. Young et al (155) sought to determine whether clinical examination characteristics could be associated with 3 sources of chronic low back pain, including 57 patients with sacroiliac joint pain. The 5 pain provocation maneuvers utilized were shown above. The authors demonstrated a significant association between a positive sacroiliac joint injection and 4 of the 9 characteristics evaluated. They also found a negative relationship between injection response and the presence of midline lumbar pain and pain above L5. 
The presence of 3 or more positive sacroiliac joint pain provocation tests was found to be significantly correlated with a positive block (phi coefficient of 0.6), resulting in an effect size of $36 \%$. They also found that all patients with a positive block experienced pain when rising from sitting, as well as a strong correlation with the non-centralization of pain.

Laslett et al (159) concluded that the diagnostic accuracy of the clinical examination in conjunction with reasoning processes was superior to sacroiliac joint pain provocation maneuvers as stand-alone tests. Excluding patients whose pain peripheralized increased the positive likelihood ratio for identifying a symptomatic sacroiliac joint(s) in patients with 3 or more provocative tests.

Laslett et al (189) also evaluated the agreement between a diagnoses reached by clinical examination and the available reference standards in a prospective study of 216 patients with lumbopelvic pain. They compared blinded clinical diagnoses by physiotherapists with diagnoses based on available reference standards for known causes of low back pain, such as discography, facet joint nerve blocks, sacroiliac joint injections, epidural injections, advanced imaging studies, or any combination of these tests. The authors found that $66 \%$ and $76 \%$ of patients received a single diagnosis based on reference standards and physical examination, respectively. Overall, exact agreement between the clinical and reference standard diagnosis was 33\%, and clinical agreement was $51 \%$. For sacroiliac joint pain, the agreement between the clinical diagnosis and the diagnosis by reference standards was $57 \%$. However, only 6 sacroiliac joint diagnoses were conferred by each method.

Broadhurst and Bond (157) also evaluated pain provocation tests for the assessment of sacroiliac joint dysfunction in a double-blind, controlled fashion. They found that local anesthetic injection suppressed approximately $70 \%$ of the pain that was elicited with provocative measures, while saline provided no meaningful relief. One flaw in this study was the large volume of local anesthetic $(4 \mathrm{~mL})$ utilized.

Maigne et al (54) determined the prevalence of sacroiliac joint pain in a selected population of patients suffering from low back pain and assessed the validity of various pain provocation tests. The patients underwent 7 sacroiliac pain provocation tests, which included the distraction test, compression test, sacral pressure test, Gaenslen test, Patrick test, resisted external rotation of the hip, and direct pressure on the pubic symphysis, before and after a screening block. No sta- tistically significant association was found between the response to the blocks and any single clinical parameter. They concluded that no pain provocation test was a useful predictor of sacroiliac joint pain.

van der Wurff et al (160) evaluated the diagnostic accuracy of a multi-test regimen of 5 sacroiliac joint pain provocation tests by comparing it to the results of fluoroscopically guided double local anesthetic blocks. The 5 provocation tests included the distraction test, compression test, thigh thrust, Patrick sign, and Gaenslen test. Among the 60 patients studied, $45 \%$ obtained a positive response to both blocks. Whereas none of the provocation tools were specific as a stand-alone test, a combination of 3 or more positive tests was deemed to be a reliable indicator. Six of the 29 patients who responded to the initial block failed to experience relief after the confirmatory block and were thus categorized as false-positive blocks. The prevalence rate for this study was $38 \%$, with a false-positive rate estimated to be around $21 \%$. The sensitivity and specificity of the 3 or more positive provocation tests were $85 \%$ and $80 \%$, respectively. The authors' conclusion that a correlation exists between the finding of 3 or more positive pain provocation tests and an analgesic response to double intraarticular sacroiliac joint blocks corroborates the results of Laslett et al (159).

Stanford and Burnham (175) evaluated provocative testing in 34 patients with suspected sacroiliac joint pain who underwent double comparative local anesthetic blocks. They found the sensitivity, specificity, and likelihood ratios for patients with 3 or more out of 6 positive tests were $0.82,0.57$, and 1.9 respectively. The provocative test utilized in this test were Patrick test, thigh thrust, ipsilateral Gaenslen test, contralateral Gaenslen test, lateral compression, and sacral thrust.

The review of provocative testing and clinical examination findings illustrates that 6 commonly performed provocative tests may be useful to select patients for further study provided 3 or more of them are positive. These include the distraction, compression, thigh thrust, Gaenslen's, and sacral thrust tests (154).

\subsection{Accuracy of Imaging}

The value of radiological imaging has been questioned in the diagnosis of sacroiliac joint pain. Multiple investigations have been performed, including evaluations using plain $x$-rays, plain CT, MRI (166-168,171, $176,194,202,206,208-210,213-237)$, single-photon emission computed tomography (SPECT) $(83,152-156,207)$ and positron emission tomography (PET) scanning 
$(211,212)$.

Among the multiple systematic reviews, Vanelderen et al (57) concluded that radiologic imaging is important to exclude "red flags" but contributes too little to aid in diagnosis. Hancock et al (49) concluded that although a positive bone scan has high specificity, it is associated with a very low sensitivity, which means the majority of patients with sacroiliac joint pain will not be accurately identified. Gupta (238) concluded that medical history, clinical examination including sacroiliac joint provocative tests, plain radiography, and laboratory investigations were helpful in diagnosing sacroiliac joint pathology in only $39 \%$ of cases, with $46 \%$ needing a CT or MRI. In a narrative review, Tuite (239) noted that the sacroiliac joint has several unique anatomical features that make it one of the more challenging joints to image, and that the radiologic findings of sacroiliitis are often equivocal. The author stated that MRI performed with proper sequencing (i.e., gadolinium enhancement or short tau inversion recovery (STIR) was the imaging modality of choice for patients with suspected sacroiliitis but negative or equivocal radiographs; however, it was unlikely to be helpful in individuals with extraarticular pathology.

The majority of the systematic reviews and individual studies conducted were related to spondyloarthritis. Thus, these results may not be applicable to chronic pain settings for evaluating sacroiliac joint pain. Several studies have also evaluated sacroiliac joint pain using various imaging techniques.

Lawson et al (235) evaluated sacroiliac joints for their anatomy with plain $\mathrm{x}$-rays and $\mathrm{CT}$ analysis. They found the accuracy of $C T$ to be superior to conventional radiography in the detection of early erosive sacroiliitis and joint space narrowing. In all patients with a discrepancy between the 2 radiologic techniques, the changes were either only or better demonstrated by CT rather than conventional radiography.

Blum et al (229) assessed plain radiographs, quantitative sacroiliac scintigraphy, and MRI. The results showed that MRI was more sensitive $(85 \%)$ than quantitative sacroiliac scintigraphy (48\%) or conventional radiography (19\%) for the detection of active sacroiliitis. For all modalities, specificity was significantly higher than sensitivity.

Jurik (240) noted that the sensitivity and specificity of conventional $x$-rays were relatively low, which can delay the diagnosis of sacroiliitis. CT was found to be superior to conventional $\mathrm{x}$-rays for the diagnosis of sacroiliitis, but this has to be weighed against the higher radiation doses utilized. The author concluded that MRI should be the imaging modality of choice for diagnosing sacroiliitis.

Puhakka et al $(202,203)$ performed 2 evaluations of MRI: one evaluated the modality in normal sacroiliac joints in asymptomatic volunteers with correlation to microscopic histology in cadavers; the second study assessed MRI abnormalities of the sacroiliac joints in early spondyloarthropathy with a one-year follow-up period. The authors concluded that coronal MRI is not conducive to the assessment of normal anatomy. But when there are variants or abnormalities of the ventral and dorsal margins of the cartilaginous sacroiliac joint and in early spondyloarthropathy, MRI can detect significant inflammatory and destructive changes over a oneyear follow-up not observable on CT or plain x-rays, despite minimal changes in the clinical parameters. Thus, MRI may be a sensitive method, with minimal risk, for the early diagnosis and evaluation for disease progression in spondyloarthropathy. Radiologic studies can also assist in determining anatomical integrity (204). A retrospective study (205) showed that CT scans were negative in $42 \%$ of patients with symptomatic sacroiliac joint pain.

In a review of imaging for spondyloarthropathy, Braun et al (230) noted that because inflammatory back pain is not a specific indicator of sacroiliitis, there is a need for imaging techniques. They concluded that scintography lacks specificity, CT is a good method to demonstrate already established bony changes, and that MRI possesses the advantage of combining good visualization of the complicated anatomy of the sacroiliac joint with the ability to localize different degrees of inflammation and edema.

Song et al (81) evaluated the diagnostic value of scintography in detecting sacroiliitis in ankylosing spondylitis and those with probable sacroiliitis without $x$-ray changes. Following an extensive literature search, they concluded that scintography of the sacroiliac joints is of limited diagnostic value.

Slipman et al (152) evaluated the value of radionuclide imaging in the diagnosis of sacroiliac joint syndrome and concluded nuclear imaging had high specificity but very low sensitivity. Consequently, they did not recommend radionuclide imaging in the evaluation of sacroiliac joint pain syndrome.

In an evaluation of quantitative radionuclide bone scanning in the diagnosis of sacroiliac joint syndrome, Maigne et al (156) found the sensitivity, specificity, and positive and negative predictive values of the quantitative bone scanning to be $46 \%, 90 \%, 86 \%$, and $72 \%$ 
respectively.

In summary, MRI appears to be a useful test in detecting early sacroiliitis, and in following disease progression in individuals with spondyloarthropathy. Imaging may also be helpful in identifying patients who might benefit from further evaluation, especially in combination with provocative maneuvers.

\subsection{Discussion}

This systematic review reveals that there continues to be relatively few high-quality studies which have investigated the diagnostic accuracy of tests to identify the sacroiliac joint(s) as the source of low back and lower extremity pain. The majority of studies investigated the role of diagnostic sacroiliac joint injections or provocation maneuvers. The results of diagnostic accuracy studies evaluating controlled local anesthetic blocks illustrate good overall evidence based on multiple high quality studies. This review indicates the approximate prevalence rate based on dual blocks to be around $25 \%$, with a false-positive rate of $20 \%$. Although provocation maneuvers have been evaluated by multiple investigators, an evaluation of these studies suggests there is limited evidence that they are helpful in determining the likelihood of sacroiliac joint pain in patients with pain located primarily below the 5 th lumbar vertebrae. Plain $\mathrm{x}$-rays or advanced imaging appear to be of no significant value in chronic sacroiliac joint pain without inflammatory arthritis.

This systematic review included 18 diagnostic accuracy studies using controlled diagnostic blocks and 12 studies evaluating provocation maneuvers. The threshold was strict in that each study had to meet at least $50 \%$ of the methodological quality assessment criteria. In contrast to our previous review (36), in this evaluation we broadened our criteria to incorporate those studies that utilized a single block and various levels of pain relief as the reference standards. We found that studies that employed single blocks, and those that used cutoff thresholds $<80 \%$, demonstrated a moderate correlation with those that utilized more stringent criteria, albeit with somewhat higher prevalence rates. There was only one placebo block available that did not provide prevalence or false-positive rates. The rationale behind using double comparative blocks is to eliminate false-positive responders, which is important to establish efficacy. The evidence for controlled dual blocks generally accepted for the diagnosis of facet joint pain $(2,35,38-40,44,87,88,241-246)$. Yet as can be seen from this review, dual blocks are not universally accepted in the interventional pain medicine community, especially when the efficacy of a procedure has already been established.

There is a paucity of literature on the effectiveness of multiple therapeutic sacroiliac joint interventional techniques including intraarticular injections or radiofrequency neurotomy of the nerve supply. Barriers to effective treatment include the fact that sacroiliac joint pain is a heterogeneous condition (i.e. patients may exhibit either intra- or extra-articular sacroiliac joint pathology) and the complex and variable nerve supply, which can make radiofrequency denervation challenging.

In a retrospective study, Borowsky and Fagen (184) evaluated the sources of sacroiliac region pain to determine the contributions of intraarticular and periarticular components. One hundred and twenty patients were evaluated with either intraarticular $(1.5 \mathrm{~mL}$ of bupivacaine and $80 \mathrm{mg}$ steroid) or a combination of intra- (2 $\mathrm{mL}$ of bupivacaine and $40 \mathrm{mg}$ steroid) and periarticular ( $2 \mathrm{~mL}$ of bupivacaine and $40 \mathrm{mg}$ steroid) injections. The periarticular injections were performed in the posterior ligaments and around the lateral branches. The authors reported a success rate of $12.5 \%$ in the intraarticular group versus $31.25 \%$ for the combined injection. Further, anesthetic response rates were also higher in the combined injection group (62.5\% versus $42.5 \%$ ). They concluded that extraarticular sources comprise of significant proportion of sacroiliac joint pain, and that performing only intraarticular diagnostic blocks may underestimate the true prevalence.

Murakami et al (187) performed a prospective study comparing intraarticular to periarticular injections, which were performed in response to pain provocation tests. The authors found that the periarticular injections effectively relieved pain in all 25 patients, but intraarticular injection was effective in only 9 of 25 patients. All 16 patients in the intraarticular group who failed to respond to the initial injection experienced significant relief after they received an injection using the periarticular approach. Overall, the $96 \%$ improvement rate after the periarticular injection was significantly higher than the $62 \%$ success rate noted after the intraarticular injection.

Dreyfuss et al $(181,182)$ evaluated the ability of single-site, single-depth, and multi-site, multi-depth sacral lateral branch blocks to anesthetize the sacroiliac joint complex. They concluded that single-site, single-depth sacral lateral branch blocks were ineffective in anesthetizing the lateral branches. In contrast, multi-site, multidepth sacral lateral branch blocks were $91 \%$ effective 
in anesthetizing the lateral branches, and blocked the pain from ligamentous probing in $70 \%$ of volunteers. However, these blocks did not block pain from the intraarticular portion of sacroiliac joint, elicited by capsular distension. This provides evidence for both a ventral and dorsal contribution to the innervation of the sacroiliac joint, and suggests that multi-side, multidepth lateral branch blocks could be useful to identify extraarticular sources of sacroiliac joint pain, and to select candidates who might benefit from lateral branch radiofrequency neurotomy. Cohen et al (116) evaluated outcome predictors for sacroiliac joint lateral branch radiofrequency denervation. Fifty-two percent of patients obtained a positive outcome, with lower baseline pain scores, pain not extending below the knee, age < 65 years, no opioid use, and the use of cooled radiofrequency being associated with success. Cohen et al (116) also evaluated radiofrequency neurotomy in a doubleblind, randomized controlled trial (68). Significantly greater pain relief and functional improvement were noted in patients who received cooled radiofrequency denervation. Based on these studies, it appears that sacroiliac joint pain is a heterogeneous condition containing both intraarticular and extraarticular components. One question that remains to be answered is how best to screen patients for denervation (i.e. intra- vs. periarticular injections; single vs. double blocks; whether or not lateral branch blocks are necessary).

The previous systematic review by Rupert et al (36) evaluating only dual blocks revealed moderate evidence for the diagnosis of sacroiliac joint pain. They estimated that sacroiliac joint pain prevalence rates ranged between $10 \%$ and $38 \%$ using a double-block paradigm, and that the false-positive rate of single, uncontrolled, sacroiliac joint injections was around $20 \%$. The authors also concluded that the evidence for provocation testing to diagnose sacroiliac joint pain was limited.

There continues to be significant debate surrounding the accuracy of diagnostic tests $(2,22$, $27,28,39,87,88,247,248)$. Although numerous instruments are available for assessing methodological quality assessment, we utilized the latest available criteria. The issues pertaining to diagnostic accuracy are somewhat contentious (35-40,44,45,85-88,138-140,249-255). The reliability of controlled comparative local anesthetic blocks has been criticized, and their validity as precision instruments questioned $(2,35,38,39,40,44,87,88,248$ $250,254-262$ ). Issues related to the validity of controlled comparative local anesthetic blocks include the quality and quantity of pain relief, the utility of dual blocks, the reference standard employed, opioid use, the effects of sedation and local anesthetic use, and the placebo effect (261-268). Whereas the evidence behind using diagnostic blocks to identify the sacroiliac joint(s) as a pain generator is not robust, one may extrapolate from some of the ample evidence in support of using controlled lumbar facet joint nerve blocks to diagnose lumbar facet joint pain $(2,35,38-40,44,49,87,88)$. The reference standard for surgical situations is clearly established by biopsy or autopsy. However, these standards are impossible to apply for pain conditions; hence, the long-term clinical follow-up of subjects appears to be the best means of establishing a reference standard with controlled blocks $(87,88)$. In a retrospective study comparing cutoff values of $50 \%$ and $80 \%$ following dual facet joint nerve blocks, Manchikanti et al (88) reported that $89.5 \%$ of patients in the $>80 \%$ group continued to have pain relief after 2-years following therapeutic medial branch blocks or radiofrequency denervation, versus $51 \%$ of patients in the $50 \%$ cutoff group. This is in contradistinction to the findings of Cohen et al, who found no differences in lumbar facet joint radiofrequency outcomes in a retrospective evaluation between those subjects who obtained $>80 \%$ relief after a single medial branch blocks and those who obtained between $50 \%$ and $80 \%$ relief (269). The study by Manchikanti et al (88) also found a lower false-positive rate at 2 years in the $>80 \%$ pain relief group. The findings by Manchikanti et al are consistent with those of other investigators who reported sustained pain relief following multiple interventions when stringent diagnostic criteria were employed $(88,241-245)$.

The use of double blocks is an area of considerable controversy. In one study, Manchikanti et al found that patients with suspected facet joint pain who tested negative using the strict criterion of $>80 \%$ pain relief following dual blocks obtained good relief following epidural steroid injections, which suggests that lowering reference standards may lead to misdiagnosis and inappropriate treatment. However, these results are in direct contrast to the findings of a randomized, comparative cost-effectiveness study published by Cohen et al (262) comparing 0,1 or 2 diagnostic blocks to select patients for lumbar facet joint radiofrequency denervation. The authors found that although the double-block group had the highest proportion of successful denervation procedures, the 0 -block group had the highest number of overall positive outcomes, and the lowest cost per successful procedure. This underscores the difference between efficacy studies, which by nature must 
employ stringent selection criteria to screen out falsepositive results and placebo responders, and those that seek to evaluate effectiveness, in which more liberal criteria should be employed. The Cohen study (262)was also conducted in a unique patient population, which may not be generalizable to other patient groups and also has been criticized for multiple deficiencies (247).

A diagnostic test is useful only to the extent that it distinguishes between the reference condition and other disorders that might otherwise be misdiagnosed. Many tests can differentiate healthy persons from severely affected ones, but being able to distinguish between these 2 groups reveals very little about the clinical utility of a test. The true pragmatic value of a test is therefore established only in a study that closely resembles clinical practice. The studies evaluating the accuracy of facet joint nerve blocks have provided us with reliability and validity data for controlled diagnostic blocks. A criterion standard using $80 \%$ pain relief during the performance of previously painful movements as the cutoff threshold following dual blocks has been established as accurate. However, due to the numerous studies that utilized less stringent criteria, we broadened the selection criteria for this review. In contrast to facet joint pain, the use of single blocks does not appear to result in unacceptably high false-positive rates, which in turn can lead to spurious prevalence estimates. The criterion standard of long-term follow-up used for the diagnosis of facet joint pain may also be applied to sacroiliac joint pain. Despite all the attention focused on establishing reference criteria, in the absence of a "gold standard" for diagnosis and treatment, any criteria utilized could be flawed (270). Although the use of multiple blocks and high cutoff thresholds will indubitably reduce the false-positive rate, an unintended consequence is that employing stricter diagnostic criteria may result in more false-negatives, the consequences of which include withholding a safe and effective treatment from a patient who might benefit - if no alternative treatment is available. Pearl (271) described a hierarchal outcome approach to test assessments using 6 criteria, which included technical aspects, diagnostic accuracy and validity, diagnostic thinking, therapeutic effectiveness, and the ability to improve patient or societal outcomes. We believe that based on long-standing experience, these criteria can be met through the use of controlled comparative local anesthetic blocks to diagnose sacroiliac joint pain.

In reference to noninvasive clinical testing for sacroiliac joint pain, since lab and imaging are non-specif- ic, the majority rely on pain provocation tests that stress the sacroiliac joint structures and provoke pain concordant with a patient's typical complaints. The key tests include distraction, compression, high thrust, Gaenslen, and sacral thrust. None of these tests have been described in sufficient detail to establish a reference standard (i.e. there is considerably variability in how these tests are performed), and many suffer from low inter-examiner reliability. Multiple other insufficiently defined tests have also been described and advocated $(53,154,155,159,189,251-253,272,273)$. Early studies reported mixed results on the interexaminer reliability of pain provocation tests evaluating sacroiliac joint pain (53). Subsequently these tests have been shown to possess acceptable levels of reliability provided that they are standardized appropriately $(53,272,274-276)$. Multiple studies with replication of results have been published $(53,154,155,159,189,251,253,272,273)$. Studies have shown that sacroiliac joint provocation tests are frequently positive in patients with other, or coexisting, sources of low back pain, such as discogenic pain, radiculopathy, and facet joint pain $(189,277)$. One of the most reliable signs of sacroiliac joint pain may be the location of pain and tenderness $(53,273)$. The centralization phenomenon is a common clinical sign observed when patients with low back pain are examined using standardized test movements and sustained postures first described by McKenzie (278). This phenomenon has been evaluated for reliability and validity in multiple contexts (279-284). Investigators have found that the centralization phenomenon is highly specific to discogenic pain, and is infrequently observed in patients with sacroiliac or facet joint pain. One may therefore assume that positive sacroiliac joint provocation tests in the presence of either centralization or a symptomatic disc herniation are likely to be falsely positive. Consequently, restricting the interpretation of sacroiliac joint provocation tests to noncentralization cases was shown to improve the specificity of 3 or more positive maneuvers from $78 \%$ to $87 \%$, without compromising sensitivity, which remained at $91 \%$ (159). Satisfying these criteria result in a high probability that sacroiliac joint pain will be confirmed by diagnostic blocks. This reasoning process may be considered a clinical prediction rule for the identification of a subset of patients most likely to have pain emanating from the sacroiliac joint region (53). Laslett (53) noted that there was a $59 \%$ probability that 3 or more positive sacroiliac joint provocation tests would lead to an ultimate diagnosis of sacroiliac joint pain, assuming that that $30 \%$ of the 
population selected will have the reference condition. If a McKenzie assessment of repeated movements fails to reveal the centralization phenomenon, there is a $77 \%$ likelihood that the pain is of sacroiliac joint origin (53). However, in most interventional pain management practices, prevalence rates of $30 \%$ are rarely seen. Consequently, the overall evidence for provocation maneuvers is limited to fair.

The limitations of this systematic review include the paucity of appropriate and high-quality literature available for analysis, the widespread variation in methodology, and the discrepancies in technical applications and reference standards. Only one placebo-controlled study was identified, which compared the effectiveness of local anesthetic blocks to intraarticular sodium chloride on the suppression of pain. However, placebo controlled injections in interventional pain management is not only impractical, but also subject to misinterpretation $(2,27,28,33,34,39,154,246,285)$.

Some investigators have asserted that any local anesthetic injection which yields similar results to steroids should be considered a placebo response; however, this interpretations may be inaccurate. The difference between injections of sodium chloride solution and dextrose into various structures has yielded varying results (246,286-292). In addition, local anesthetics have been shown to provide long-term relief (293-296) in both clinical and experimental settings. Thus, local comparative anesthetic blocks appear may be more practical than placebo-controlled injections unless the latter are applied and interpreted appropriately. Ghahreman and Bogduk (297) illustrated the proper application of a placebo in a clinical trial evaluating transforaminal epidural steroid injections, which can be extrapolated in a similar fashion for sacroiliac joint injections.

The results of this systematic review may be used in future research to identify patients more likely to have pain originating from the sacroiliac joint, as well as to evaluate the effectiveness of therapeutic interventions targeting sacroiliac joint pain, but the conclusions are only as robust as the accuracy of the studies analyzed. As noted earlier, there continues to be considerable controversy surrounding the use of diagnostic controlled local anesthetic blocks.
In summary, sacroiliac joint injections are safe and reasonable tools when used for diagnosis after properly selecting candidates based on provocative maneuvers.

\subsection{Conclusion}

The results of this systematic review demonstrate fair to good support for diagnostic interventions using dual local anesthetic blocks in patients positive for sacroiliac joint provocation maneuvers who have failed conservative management.

\section{Author Affiliations}

Dr. Simopoulos is Assistant Professor, Department of Anesthesia, Critical Care, and Pain Medicine, Beth Israel Deaconess Medical Center, Harvard Medical School, Boston, MA.

Dr. Manchikanti is Medical Director of the Pain Management Center of Paducah, Paducah, KY, and Clinical Professor, Anesthesiology and Perioperative Medicine, University of Louisville, Louisville, KY.

Dr. Singh is Medical Director, Pain Diagnostics Associates, Niagara, WI.

Dr. Gupta is a Consultant in Pain Medicine and Anaesthesia, Bradford Teaching Hospital NHS Foundation Trust, Bradford Royal Infirmary, Bradford, United Kingdom.

Dr. Hameed is with the Department of Physical Medicine and Rehabilitation, The Johns Hopkins University School of Medicine, Baltimore, MD.

Dr. Diwan is Executive Director of The Spine and Pain Institute of New York.

Dr. Cohen is Associate Professor, Department of Anesthesiology and Critical Care Medicine, Pain Management Division, Johns Hopkins School of Medicine, Baltimore, MD, and Associate Professor, Department of Anesthesiology, Uniformed Services University of the Health Sciences, Bethesda, MD.

\section{Acknowledgments}

The authors wish to thank Vidyasagar Pampati, MSc, for statistical assistance; Sekar Edem for assistance in the search of the literature; Bert Fellows, MA, and Tom Prigge, MA, for manuscript review; and Tonie M. Hatton and Diane E. Neihoff, transcriptionists, for their assistance in preparation of this manuscript. We would like to thank the editorial board of Pain Physician for review and criticism in improving the manuscript. 


\section{References}

1. Pizzo PA, Clark NM. Alleviating suffering 101 - pain relief in the United States. N Engl] Med 2012; 366:197-199.

2. Manchikanti L, Boswell MV, Singh $V$, Benyamin RM, Fellows B, Abdi S, Buenaventura RM, Conn A, Datta S, Derby R, Falco FJE, Erhart S, Diwan S, Hayek SM, Helm S, Parr AT, Schultz DM, Smith HS, Wolfer LR, Hirsch JA. Comprehensive evidence-based guidelines for interventional techniques in the management of chronic spinal pain. Pain Physician 2009; 12:699-802.

3. Martin BI, Turner JA, Mirza SK, Lee M), Comstock BA, Deyo RA. Trends in health care expenditures, utilization, and health status among US adults with spine problems, 1997-2006. Spine (Phila Pa 1976) 2009; 34:2077-2084.

4. Hoy D, Brooks P, Blyth F, Buchbinder R. The epidemiology of low back pain. Best Pract Res Clin Rheumatol 2010; 24:769781.

5. Institute of Medicine (IOM). Relieving Pain in America: A Blueprint for Transforming Prevention, Care, Education, and Research. The National Academies Press, Washington, DC, June 29, 2011. www.iom.edu/ /media/Files/Report\%20Files/2011/Relieving-Pain-inAmerica-A-Blueprint-for-TransformingPrevention-Care-Education-Research/ Pain\%20Research\%202011\%20Report\%2oBrief.pdf

6. Verhaak PF, Kerssens JJ, Dekker J, Sorbi MJ, Bensing JM. Prevalence of chronic benign pain disorder among adults: A review of the literature. Pain 1998; 77:231-239.

7. Elliott AM, Smith BH, Hannaford PC, Smith WC, Chambers WA. The course of chronic pain in the community: Results of a 4-year follow-up study. Pain 2002; 99:299-307.

8. Cassidy JD, Carroll LJ, Cotê P. The Saskatchewan Health and Back Pain Survey. The prevalence of low back pain and related disability in Saskatchewan adults. Spine (Phila Pa 1976) 1998; 23:1860-1867.

9. Freburger JK, Holmes GM, Agans RP, Jackman AM, Darter JD, Wallace AS, Castel LD, Kalsbeek WD, Carey TS. The rising prevalence of chronic low back pain. Arch Intern Med 2009; 169:251-258.

10. Bressler HB, Keyes WJ, Rochon PA, Badley $E$. The prevalence of low back pain in the elderly. A systematic review of the literature. Spine (Phila Pa 1976) 1999; 24:1813-1819.

11. Manchikanti L, Singh V, Datta S, Cohen
SP, Hirsch JA. Comprehensive review of epidemiology, scope, and impact of spinal pain. Pain Physician 2009; 12:E35-E70.

12. Luo X, Pietrobon R, Sun SX, Liu GG, Hey L. Estimates and patterns of direct health care expenditures among individuals with back pain in the United States. Spine (Phila Pa 1976) 2004; 29:79-86.

13. Walker BF, Muller R, Grant WD. Low back pain in Australian adults: The economic burden. Asia Pac J Public Health 2003; 15:79-87.

14. Deyo RA, Mirza SK, Turner JA, Martin BI. Overtreating chronic back pain: Time to back off? J Am Board Fam Med 2009; 22:62-68.

15. Ivanova Jl, Birnbaum HG, Schiller M, Kantor E, Johnstone BM, Swindle RW. Real-world practice patterns, health-care utilization, and costs in patients with low back pain: The long road to guidelineconcordant care. Spine ] 2011; 11:622-632.

16. Manchikanti L, Pampati V, Boswell MV, Smith HS, Hirsch JA. Analysis of the growth of epidural injections and costs in the Medicare population: A comparative evaluation of 1997, 2002, and 2006 data. Pain Physician 2010; 13:199-212.

17. Staal JB, de Bie RA, de Vet HC, Hildebrandt J, Nelemans P. Injection therapy for subacute and chronic low back pain: An updated Cochrane review. Spine (Phila Pa 1976) 2009; 34:49-59.

18. Manchikanti L, Fellows B, Ailinani $\mathrm{H}$, Pampati V. Therapeutic use, abuse, and nonmedical use of opioids: A ten-year perspective. Pain Physician 2010; 13:401435 .

19. Manchikanti L, Ailinani H, Koyyalagunta D, Datta S, Singh V, Eriator I, Sehgal N, Shah RV, Benyamin RM, Vallejo R, Fellows B, Christo PJ. A systematic review of randomized trials of long-term opioid management for chronic non-cancer pain. Pain Physician 2011; 14:91-121.

20. Manchikanti L, Vallejo R, Manchikanti KN, Benyamin RM, Datta S, Christo PJ. Effectiveness of long-term opioid therapy for chronic non-cancer pain. Pain Physician 2011; 14: E133-E156.

21. Jacobs WC, van Tulder M, Arts M, Rubinstein SM, van Middelkoop M, Ostelo R, Verhagen A, Koes B, Peul WC. Surgery versus conservative management of sciatica due to a lumbar herniated disc: A systematic review. Eur Spine J 2011; 20:513-522.

22. Chou R, Huffman L. Guideline for the Evaluation and Management of Low Back Pain: Evidence Review. American Pain So- ciety, Glenview, IL, 2009. www.ampainsoc.org/pub/pdf/LBPEvidRev.pdf

23. Chou R, Huffman L. Use of Chronic Opioid Therapy in Chronic Noncancer Pain: Evidence Review. American Pain Society; Glenview, IL: 2009.

www.ampainsoc.org/pub/pdf/Opioid_ Final_Evidence_Report.pdf

24. Abbott ZI, Nair KV, Allen RR, Akuthota VR. Utilization characteristics of spinal interventions. Spine ] 2012; 1:35-43.

25. Manchikanti L, Pampati V, Singh V, Boswell MV, Smith HS, Hirsch JA. Explosive growth of facet joint interventions in the Medicare population in the United States: A comparative evaluation of 1997, 2002, and 2006 data. BMC Health Serv Res 2010; 10:84.

26. Manchikanti L, Singh V, Caraway DL, Benyamin RM, Hirsch JA. Medicare physician payment systems: Impact of 2011 schedule on interventional pain management. Pain Physician 2011; 14:E5-E33.

27. Manchikanti L, Datta S, Derby R, Wolfer LR, Benyamin RM, Hirsch JA. A critical review of the American Pain Society clinical practice guidelines for interventional techniques: Part 1. Diagnostic interventions. Pain Physician 2010; 13:E141E174.

28. Manchikanti L, Datta S, Gupta S, Munglani R, Bryce DA, Ward SP, Benyamin RM, Sharma ML, Helm II S, Fellows B, Hirsch JA. A critical review of the American Pain Society clinical practice guidelines for interventional techniques: Part 2. Therapeutic interventions. Pain Physician 2010; 13:E215-E264.

29. Tosteson AN, Tosteson TD, Lurie JD, Abdu W, Herkowitz H, Andersson G, Albert T, Bridwell K, Zhao W, Grove MR, Weinstein MC, Weinstein JN. Comparative effectiveness evidence from the spine patient outcomes research trial: Surgical versus nonoperative care for spinal stenosis, degenerative spondylolisthesis, and intervertebral disc herniation. Spine (Phila Pa 1976) 2011; 36:20612068.

30. Rubinstein SM, van Middelkoop M, Assendelft WJ, de Boer MR, van Tulder MW. Spinal manipulative therapy for chronic low-back pain: An update of a Cochrane review. Spine (Phila Pa 1976) 2011; 36:E825-E846.

31. Hahne AJ, Ford JJ, McMeeken JM. Conservative management of lumbar disc herniation with associated radiculopathy: A systematic review. 
Spine (Phila Pa 1976) 2010; 35:E488-E504.

32. van Middelkoop M, Rubinstein SM, Kuijpers T, Verhagen AP, Ostelo R, Koes BW, van Tulder MW. A systematic review on the effectiveness of physical and rehabilitation interventions for chronic nonspecific low back pain. Eur Spine J 2011; 20:19-39.

33. Manchikanti L, Falco FJE, Boswell MV, Hirsch JA. Facts, fallacies, and politics of comparative effectiveness research: Part 1. Basic considerations. Pain Physician 2010; 13:E23-E54.

34. Manchikanti L, Falco FJE, Boswell MV, Hirsch JA. Facts, fallacies, and politics of comparative effectiveness research: Part 2. Implications for interventional pain management. Pain Physician 2010; 13:E55-E79.

35. Falco FJE, Erhart S, Wargo BW, Bryce DA, Atluri S, Datta S, Hayek SM. Systematic review of diagnostic utility and therapeutic effectiveness of cervical facet joint interventions. Pain Physician 2009; 12:323-344.

36. Rupert MP, Lee M, Manchikanti L, Datta S, Cohen SP. Evaluation of sacroiliac joint interventions: A systematic appraisal of the literature. Pain Physician 2009; 12:399-418.

37. Manchikanti L, Glaser S, Wolfer L, Derby R, Cohen SP. Systematic review of lumbar discography as a diagnostic test for chronic low back pain. Pain Physician 2009; 12:541-559.

38. Datta S, Lee M, Falco FJE, Bryce DA, Hayek SM. Systematic assessment of diagnostic accuracy and therapeutic utility of lumbar facet joint interventions. Pain Physician 2009; 12:437-460.

39. Manchikanti L, Boswell MV, Singh V, Derby R, Fellows B, Falco FJE, Datta $S$, Smith HS, Hirsch JA. Comprehensive review of neurophysiologic basis and diagnostic interventions in managing chronic spinal pain. Pain Physician 2009; 12:E71-E120.

40. Bogduk N. International spinal injection society guidelines for the performance of spinal injection procedures. Part 1. Zygapophysial joint blocks. Clin J Pain 1997; 13:285-302.

41. Manchikanti L, Singh V, Pampati V, Damron K, Barnhill R, Beyer C, Cash K. Evaluation of the relative contributions of various structures in chronic low back pain. Pain Physician 2001; 4:308-316.

42. Pang WW, Mok MS, Lin ML, Chang DP, Hwang $\mathrm{MH}$. Application of spinal pain mapping in the diagnosis of low back pain--analysis of 104 cases. Acta Anaesthesiol Sin 1998; 36:71-74.

43. Bogduk N. Low back pain. In: Clinical Anatomy of the Lumbar Spine and Sacrum. 4th edition. Churchill Livingstone, New York, 2005, pp 183-216.

44. Rubinstein SM, van Tulder M. A best-evidence review of diagnostic procedures for neck and low-back pain. Best Pract Res Clin Rheumatol 2008; 22:471-482.

45. Wolfer L, Derby R, Lee JE, Lee SH. Systematic review of lumbar provocation discography in asymptomatic subjects with a meta-analysis of false-positive rates. Pain Physician 2008; 11:513-538.

46. Cohen SP. Sacroiliac joint pain: A comprehensive review of anatomy, diagnosis and treatment. Anesth Analg 2005; 101:1440-1453.

47. Bogduk N. The sacroiliac joint. In: Clinical Anatomy of Lumbar Spine and Sacrum, 4th edition. Churchill Livingstone, New York, 2005 pp 173-181.

48. Merskey H, Bogduk N. Sacroiliac joint pain in Group XXVII: Sacral spinal or radicular pain syndromes. In: Classification of Chronic Pain: Descriptions of Chronic Pain Syndromes and Definition of Pain Terms, 2nd ed. Task Force on Taxonomy of the International Association for the Study of Pain. IASP Press, Seattle, 1994, pp 190-191.

49. Hancock MJ, Maher CG, Latimer J, Spindler MF, McAuley JH, Laslett M, Bogduk N. Systematic review of tests to identify the disc, SIJ or facet joint as the source of low back pain. Eur Spine ] 2007; 16:1539-1550.

50. Foley BS, Buschbacher RM. Sacroiliac joint pain: Anatomy, biomechanics, diagnosis, and treatment. Am J Phys Med Rehabil 2006; 85:997-1006.

51. Forst SL, Wheeler MT, Fortin JD, Vilensky JA. The sacroiliac joint: Anatomy, physiology, and clinical significance. Pain Physician 2006; 9:61-67.

52. Zelle BA, Gruen GS, Brown S, George S. Sacroiliac joint dysfunction: Evaluation and management. Clin J Pain 2005; 21:446-455.

53. Laslett M. Evidence-based diagnosis and treatment of the painful sacroiliac joint. J Man Manip Ther 2008; 16:142-152.

54. Maigne JY, Aivakiklis A, Pfefer F. Results of sacroiliac joint double block and value of sacroiliac pain provocation test in 54 patients with low back pain. Spine (Phila Pa 1976) 1996; 21:1889-1892.

55. Irwin RW, Watson T, Minick RP, Ambrosius WT. Age, body mass index, and gender differences in sacroiliac joint pathology. Am J Phys Med Rehabil 2007; 86:37-44.

56. Peterson C, Hodler J. Evidence-based radiology (part 1): Is there sufficient research to support the use of therapeutic injections for the spine and sacroiliac joints? Skeletal Radiol 2010; 39:5-9.

57. Vanelderen P, Szadek K, Cohen SP, De Witte J, Lataster A, Patijn J, Mekhail N, van Kleef M, Van Zundert J. Sacroiliac joint pain. Pain Pract 2010; 10:470-478.

58. Muhlner SB. Review article: Radiofrequency neurotomy for the treatment of sacroiliac joint syndrome. Curr Rev Musculoskelet Med 2009; 2:10-14.

59. Speldewinde GC. Outcomes of percutaneous zygapophysial and sacroiliac joint neurotomy in a community setting. Pain Med 2011; 12:209-218.

6o. Hawkins J, Schofferman J. Serial therapeutic sacroiliac joint injections: A practice audit. Pain Med 2009; 10:850-853.

61. Kennedy DJ, Shokat M, Visco CJ. Sacroiliac joint and lumbar zygapophysial joint corticosteroid injections. Phys Med Rehabil Clin N Am 2010; 21:835-842.

62. Ferrante FM, King LF, Roche EA, Kim PS, Aranda M, Delaney LR, Mardini IA, Mannes AJ. Radiofrequency sacroiliac joint denervation for sacroiliac syndrome. Reg Anesth Pain Med 2001; 26:137-142.

63. Dussault RG, Kaplan PA, Anderson MW. Fluoroscopy-guided sacroiliac joint injections. Radiology 2000; 214:273-277.

64. Buijs EJ, Kamphuis ET, Groen GJ. Radiofrequency treatment of sacroiliac jointrelated pain aimed at the first three sacral dorsal rami: A minimal approach. Pain Clinic 2004; 16:139-146.

65. Buchowski JM, Kebaish KM, Sinkov V, Cohen DB, Sieber AN, Kostuik JP. Functional and radiographic outcome of sacroiliac arthrodesis for the disorders of the sacroiliac joint. Spine ] 2005; 5:520528; discussion 529.

66. Vallejo R, Benyamin RM, Kramer J, Stanton G, Joseph NJ. Pulsed radiofrequency denervation for the treatment of sacroiliac joint syndrome. Pain Med 2006; 7:429-434.

67. Burnham RS, Yasui Y. An alternate method of radiofrequency neurotomy of the sacroiliac joint: A pilot study of the effect on pain, function, and satisfaction. Reg Anesth Pain Med 2007; 32:1219.

68. Cohen SP, Hurley RW, Buckenmaier CC 3rd, Kurihara C, Morlando B, Dragov- 
ich A. Randomized placebo-controlled study evaluating lateral branch radiofrequency denervation for sacroiliac joint pain. Anesthesiology 2008; 109:279-288.

69. Al-Khayer A, Hegarty J, Hahn D, Grevitt MP. Percutaneous sacroiliac joint arthrodesis: A novel technique. J Spinal Disord Tech 2008; 21:359-363.

70. Amoretti N, Hovorka I, Marcy PY, Hauger O, Amoretti ME, Lesbats V, Brunner P, Maratos Y, Stedman S, Boileau P. Computed axial tomographyguided fixation of sacroiliac joint disruption: Safety, outcomes, and results at 3-year follow-up. Cardiovasc Intervent Radiol 2009; 32:1227-1234.

71. Liliang PC, Lu K, Weng HC, Liang CL, Tsai YD, Chen HJ. The therapeutic efficacy of sacroiliac joint blocks with triamcinolone acetonide in the treatment of sacroiliac joint dysfunction without spondyloarthropathy. Spine (Phila $\mathrm{Pa}$ 1976) 2009; 34:896-900.

72. Lucas N, Macaskill P, Irwig L, Moran R, Bogduk N. Reliability of physical examination for diagnosis of myofascial trigger points: A systematic review of the literature. Clin J Pain 2009; 25:80-89.

73. Lucas NP, Macaskill P, Irwig L, Bogduk $\mathrm{N}$. The development of a quality appraisal tool for studies of diagnostic reliability (QAREL). J Clin Epidemiol 2010; 63:854-861.

74. Manchikanti L, Derby R, Wolfer LR, Singh V, Datta S, Hirsch JA. Evidencebased medicine, systematic reviews, and guidelines in interventional pain management: Part 5. Diagnostic accuracy studies. Pain Physician 2009; 12:517-540.

75. Manchikanti L, Derby R, Wolfer LR, Singh V, Datta S, Hirsch JA. Evidencebased medicine, systematic reviews, and guidelines in interventional pain management: Part 7: Systematic reviews and meta-analyses of diagnostic accuracy studies. Pain Physician 2009; 12:929-963.

76. Bossuyt PM, Reitsma JB, Bruns DE, Gatsonis CA, Glasziou PP, Irwig LM, Lijmer JG, Moher D, Rennie D, de Vet HC; STARD Group. Towards complete and accurate reporting of studies of diagnostic accuracy: The STARD Initiative. Ann Intern Med 2003; 138:40-44.

77. Whiting $P$, Rutjes AW, Reitsma JB, Bossuyt PM, Kleijnen J. The development of QUADAS: A tool for the quality assessment of studies of diagnostic accuracy included in systematic reviews. BMC Med Res Methodol 2003; 3:25.

78. DePalma MJ, Ketchum JM, Saullo T.
What is the source of chronic low back pain and does age play a role? Pain Med 2011; 12:224-233.

79. DePalma M, Ketchum J, Saullo T, Schofferman J. Structural etiology of chronic low back pain due to motor vehicle collision. Pain Med 2011; 12:1622-1627.

8o. Szadek KM, van der Wurff P, van Tulder MW, Zuurmond WW, Perez RR. Diagnostic validity of criteria for sacroiliac joint pain: A systematic review. J Pain 2008; 10:354-368.

81. Song $\mathrm{IH}$, Carrasco-Fernández J, Rudwaleit $M$, Sieper J. The diagnostic value of scintigraphy in assessing sacroiliitis in ankylosing spondylitis: A systematic literature research. Ann Rheum Dis 2008; 67:1535-1540.

82. Schwarzer AC, Aprill CN, Bogduk M. The sacroiliac joint in chronic low back pain. Spine (Phila Pa 1976) 1995; 20:31-37.

83. Maigne JY, Planchon CA. Sacroiliac joint pain after fusion. A study with anesthetic blocks. Eur Spine ] 2005; 14:654-658.

84. Manchikanti L, Singh V, Derby R, Schultz DM, Benyamin RM, Prager JP, Hirsch JA. Reassessment of evidence synthesis of occupational medicine practice guidelines for interventional pain management. Pain Physician 2008; 11:393482.

85. Carragee EJ, Lincoln TF, Parmar VS, Alamin T. A gold standard evaluation of the "discogenic pain" diagnosis as determined by provocative discography. Spine (Phila Pa 1976) 2006; 31:2115-2123.

86. Carragee EJ, Haldeman S, Hurtwitz E. The pyrite standard: The Midas touch in the diagnosis of axial pain syndromes. Spine J 2007; 7:27-31.

87. Pampati S, Cash KA, Manchikanti L. Accuracy of diagnostic lumbar facet joint nerve blocks: A 2-year follow-up of 152 patients diagnosed with controlled diagnostic blocks. Pain Physician 2009; 12:855-866.

88. Manchikanti L, Pampati S, Cash KA. Making sense of the accuracy of diagnostic lumbar facet joint nerve blocks: An assessment of implications of $50 \%$ relief, $80 \%$ relief, single block or controlled diagnostic blocks. Pain Physician 2010; 13:133-143.

89. Berthelot JM, Labat JJ, Le Goff B, Gouin F, Maugars Y. Provocative sacroiliac joint maneuvers and sacroiliac joint block are unreliable for diagnosing sacroiliac joint pain. Joint Bone Spine 2006; 73:17-23.

90. Fortin JD. The sacroiliac joint: A new perspective. J Back Muskuloskel Rehabil
1993; 3:31-43.

91. Fortin J, Sehgal N. Sacroiliac joint injection and arthrography with imaging correlation. In: Lennard TA (ed). Pain Procedures in Clinical Practice. Hanley \& Belfus, Philadelphia, 2000, pp 265-273.

92. Smidt GL, Wei S, McQuade K. Sacroiliac motion for extreme hip positions. Spine (Phila Pa 1976) 1997; 22:2073-2082.

93. Bowen V, Cassidy JD. Macroscopic and microscopic anatomy of the sacroiliac joint from embryonic life until the eighth decade. Spine (Phila Pa 1976) 1981; 6:620-628.

94. Brooke R. The sacroiliac joint. J Anat 1924; 58:299-305.

95. Gunterberg B, Romanus B, Stener B. Pelvic strength after major amputation of the sacrum: An experimental approach. Acta Orthop Scand 1976; 47:635642.

96. Miller JAA, Schultz AM, Anderson GISJ. Loading displacement behaviors of sacroiliac joints. J Orthop Res 1987; 5:92-101.

97. Cunningham DJ. Cunningham's TextBook of Anatomy. Oxford University Press, New York, 1931.

98. Solonen KA. The sacroiliac joint in the light of anatomical, roentgenological and clinical studies. Acta Orthop Scand Suppl 1957; 27:1-127.

99. Vilensky JA, O'Connor BL, Fortin JD, Merkel GJ, Jimenez AM, Scofield BA, Kleiner JB. Histologic analysis of neural elements in the human sacroiliac joint. Spine (Phila Pa 1976) 2002; 27:1202-1207.

100. Ikeda R. Innervation of the sacroiliac joint. Macroscopical and histological studies. Nippon Ika Daigaku Zasshi 1991; 58:587-596.

101. Murata Y, Takahashi K, Yamagata M, Takahashi Y, Shimada Y, Moriya H. Sensory innervation of the sacroiliac joint in rats. Spine (Phila Pa 1976) 2000; 25:20152019.

102. Umimura T, Miyagi M, Ishikawa T, Kamoda H, Wakai K, Sakuma T, Sakai R, Kuniyoshi K, Ochiai N, Kishida S, Nakamura J, Eguchi Y, Iwakura N, Kenmoku T, Arai G, Orita S, Suzuki M, Sakuma Y, Kubota G, Oikawa Y, Inoue G, Aoki Y, Toyone T, Takahashi K, Ohtori S. Investigation of dichotomizing sensory nerve fibers projecting to the lumbar multifidus muscles and intervertebral disk or facet joint or sacroiliac joint in rats. Spine (Phila Pa 1976) 2011 Jun 20. [Epub ahead of print].

103. Sakamoto N, Yamashita T, Takebayashi T, Sekine M, Ishii S. An electrophys- 
iologic study of mechanoreceptors in the sacroiliac joint and adjacent tissues. Spine (Phila Pa 1976) 2001; 26:E468-E471.

104. Grob KR, Neuhuber WL, Kissling RO. Innervation of the sacroiliac joint of the human. Z Rheumatol 1995; 54:117-122.

105. Szadek KM, Hoogland PV, Zuurmond WW, de Lange J, Perez RS. Nociceptive nerve fibers in the sacroiliac joint in humans. Reg Anesth Pain Med 2008; 33:3643.

106. Holm S, Indahl A, Solomonow M. Sensorimotor control of the spine. J Electromyogr Kinesiol 2002;12:219-234.

107. Fortin JD, Kissling RO, O'Connor BL, Vilensky JA. Sacroiliac joint innervation and pain. Am J Orthop 1999; 28:687-69o.

108. Bernard TN Jr. The sacroiliac joint syndrome. Pathophysiology, diagnosis, and management. In: Frymoyer JW (ed). The Adult Spine. Principles and Practice. Raven Press, New York, 1991, pp 2107-2130.

109. Nakagawa T. A study on the distribution of nerve filaments of the iliosacral joint and its adjacent region in the Japanese. J Jap Orthop Assoc 1966; 40:419-430.

110. Kiter E, Karaboyun T, Tufan AC, Acar K. Immunohistochemical demonstration of nerve endings in iliolumbar ligament. Spine (Phila Pa 1976) 2010; 35:E101-E104.

111. Fortin JD, Vilensky JA, Merkel GJ. Can the sacroiliac joint cause sciatica? Pain Physician 2003; 6:269-271.

112. McGrath MC, Zhang M. Lateral branches of dorsal sacral nerve plexus and the long posterior sacroiliac ligament. Surg Radiol Anat 2005; 27:327-330.

113. Vilensky J. Innervation of the joint and its role in osteoarthritis. In: Brandt $\mathrm{K}$, Doherty M, Lohamander L (eds.), Osteoarthritis. Oxford University Press, Oxford, 1998, pp 176-188.

114. Fortin JD, Falco F, Washington W. Three pathways between the sacroiliac joint and neural structures exist. Am J Neuroradial 1999; 20:1429-1434.

115. Chou LH, Slipman CW, Bhagia SM, Tsaur L, Bhat AL, Isaac Z, Gilchrist R, El Abd OH, Lenrow DA. Inciting events initiating injection-proven sacroiliac joint syndrome. Pain Med 2004; 5:26-32.

116. Cohen SP, Strassels SA, Kurihara C, Crooks MT, Erdek MA, Forsythe A, Marcuson M. Outcome predictors for sacroiliac joint (lateral branch) radiofrequency denervation. Reg Anesth Pain Med 2009; 34:206-214

117. Ha KY, Lee JS, Kim KW. Degeneration of sacroiliac joint after instrumented lumbar or lumbosacral fusion: A prospective cohort study over five-year follow-up. Spine (Phila Pa 1976) 2008; 33:1192-1198.

118. Ivanov AA, Kiapour A, Ebraheim NA, Goel V. Lumbar fusion leads to increases in angular motion and stress across sacroiliac joint: A finite element study. Spine (Phila Pa 1976) 2009; 34:E162-E169.

119. DePalma MJ, Ketchum JM, Saullo TR. Etiology of chronic low back pain in patients having undergone lumbar fusion. Pain Med 2011; 12:732-739.

120. Zhang Q, Chen W, Liu H, Su Y, Pan J, Zhang Y. The anterior dislocation of the sacroiliac joint: A report of four cases and review of the literature and treatment algorism. Arch Orthop Trauma Surg 2009; 129:941-947.

121. O'Shea FD, Boyle E, Salonen DC, Ammendolia C, Peterson C, Hsu W, Inman RD. Inflammatory and degenerative sacroiliac joint disease in a primary back pain cohort. Arthritis Care Res (Hoboken) 2010; 62:447-454.

122. Yilmaz N, Ozgocmen S, Kocakoc E, Kiris A. Primary hydatid disease of sacrum affecting the sacroiliac joint: A case report. Spine (Phila Pa 1976) 2004; 29:E88-E90; discussion E91.

123. DePalma MJ, Ketchum JM, Saulio TR Laplante BL. Is the history of a surgical discectomy related to the source of chronic low back pain? Pain Physician 2012: 15:E1-E6.

124. Ha KY, Lee JS, Kim KW. Degeneration of sacroiliac joint after instrumented lumbar or lumbosacral fusion: A prospective cohort study over five-year follow-up. Spine (Phila Pa 1976) 2008; 33:1192-1198.

125. Hansen HC, McKenzie-Brown AM, Cohen SP, Swicegood JR, Colson JD, Manchikanti L. Sacroiliac joint interventions: A systematic review. Pain Physician 2007; 10:165-184.

126. McKenzie-Brown AM, Shah RV, Sehga N, Everett CR. A systematic review of sacroiliac joint interventions. Pain Physician 2005; 8:115-125.

127. Liberati A, Altman DG, Tetzlaff J, Mulrow C, Gøtzsche PC, loannidis JP, Clarke M, Devereaux PJ, Kleijnen J, Moher D. The PRISMA statement for reporting systematic reviews and meta-analyses of studies that evaluate health care interventions: Explanation and elaboration. Ann Intern Med 2009; 151:W65-W94.

128. Whiting PF, Rutjes AW, Westwood ME, Mallett S, Deeks JJ, Reitsma JB, Leeflang MM, Sterne JA, Bossuyt PM; QUADAS-2 Group. QUADAS-2: A revised tool for the quality assessment of diagnostic accuracy studies. Ann Intern Med 2011; 155:529-
536.

129. Gemmell H, Miller P. Interexaminer reliability of multidimensional examination regimens used for detecting spinal manipulable lesions: A systematic review. Clin Chiropractic 2005; 8:199-204.

130. Hestboek L, Leboeuf-Yde C. Are chiropractic tests for the lumbo-pelvic spine reliable and valid? A systematic critical literature review. J Manipulative Physiol Ther 2000; 23:258-275.

131. Hollerwoger D. Methodological quality and outcomes of studies addressing manual cervical spine examinations: A review. Man Ther 2006; 11:93-98.

132. May S, Littlewook C, Bishop A. Reliability of procedures used in the physical examination of non-specific low back pain: A systematic review. Aust J Physiother 2006; 52:91-102.

133. Stochkendahl MJ, Christensen HW, Hartvigsen J, Vach W, Haas M, Hestbaek L, Adams A, Bronfort G. Manual examination of the spine: A systematic critical literature review of reproducibility. J Manipulative Physiol Ther 2006; 29:475-485, 485.e1-10.

134. van Trijffel E, Anderegg Q, Bossuyt PM, Lucas C. Inter-examiner reliability of passive assessment of intervertebral motion in the cervical and lumbar spine: A systematic review. Man Ther 2005; 10:256-269.

135. van Tulder M, Furlan A, Bombardier C, Bouter L; Editorial Board of the Cochrane Collaboration Back Review Group. Updated method guidelines for systematic reviews in the Cochrane Collaboration Back Review Group. Spine (Phila Pa 1976) 2003; 28:1290-1299.

136. Staal JB, de Bie R, de Vet HC, Hildebrandt J, Nelemans P. Injection therapy for subacute and chronic low-back pain. Cochrane Database Syst Rev 2008; 3:CDool824.

137. Harbord R, Higgins J. METAREG: Stata module to perform meta-analysis regression. Boston College Dept of Econ, Boston, MA. www.econpapers.org/software/ bocbocode/s446201.htm

138. Harris RP, Helfand M, Woolf SH, Lohr KN, Mulrow CD, Teutsch SM, Atkins D; Methods Work Group, Third US Preventive Services Task Force. Current methods of the US Preventive Services Task Force. Am J Prevent Med 2001; 20:21-35.

139. Manchikanti L, Benyamin RM, Singh V, Bryce DA, Hameed H, Derby R, Cohen S. Systematic appraisal of accuracy of utility of lumbar discography in chron- 
ic low back pain. Pain Physician 2012; in press.

140. Singh V, Chiravuri S, Falco FJE, Manchikant L, Onyewu O, Ruan X. Systematic review of accuracy of utility of thoracic discography as a diagnostic test for chronic spinal pain. Pain Physician 2012; in press.

141. Onyewu O, Manchikanti L, Christo PJ, Cohen S, Falco FJE, Geffert S, Helm S, Simopoulos TT, Hameed M. Systematic review of cervical discography as a diagnostic test for chronic spinal pain. Pain Physician 2012; in press.

142. Sehgal N, Chiravuri S, Datta S, Falco FJE, Geffert S, Onyewu O. Systematic assessment of diagnostic accuracy of lumbar facet joint interventions. Pain Physician 2012; in press.

143. Datta S, Chiravuri S, Falco FJE, Geffert S, Sehgal N. Systematic review of diagnostic utility of thoracic facet joint interventions. Pain Physician 2012; in press.

144. Datta S, Chiravuri S, Falco FJE, Gefferrt $S$, Ruan X, Sehgal N. Systematic review of diagnostic utility of cervical facet joint interventions. Pain Physician 2012; in press.

145. Benyamin RM, Manchikanti L, Parr AT, Diwan S, Abdi S. The effectiveness of lumbar interlaminar epidural injections in managing chronic low back and lower extremity pain. Pain Physician 2012; in press.

146. Parr AT, Manchikanti L, Hameed H, Conn A, Manchikanti KN, Benyamin RM, Diwan S, Singh V, Abdi S. Caudal epidural injections in the management of chronic low back pain: A systematic appraisal of the literature. Pain Physician 2012; 15:159-198.

147. Diwan S, Benyamin RM, Bryce D, Falco FJE, Geffert S, Hameed H, Sharma M Abdi S. Effectiveness of cervical epidurals in the management of chronic neck and upper extremity pain. Pain Physician 2012; In Press.

148. Dreyfuss $P$, Michaelsen M, Pauza K, McLarty J, Bogduk N. The value of medical history and physical examination in diagnosing sacroiliac joint pain. Spine (Phila Pa 1976) 1996; 21:2594-2602.

149. Katz V, Schofferman J, Reynolds J. The sacroiliac joint: A potential cause of pain after lumbar fusion to the sacrum. J Spinal Disord Tech 2003; 16:96-99.

150. Slipman CW, Sterenfeld EB, Chou LH, Herzog R, Vresilovic E. The predictive value of provocative sacroiliac joint stress maneuvers in the diagnosis of sacroiliac joint syndrome. Arch Phys Med Rehabil 1998; 79:288-292.

151. Heuft-Dorenbosch L, Landewé R, Weijers $\mathrm{R}$, Wanders A, Houben $\mathrm{H}$, van der Linden S, van der Heijde D. Combining information obtained from magnetic resonance imaging and conventional radiographs to detect sacroiliitis in patients with recent onset inflammatory back pain. Ann Rheum Dis 2006; 65:804808.

152. Slipman CW, Sterenfeld EB, Chou LH, Herzog R, Vresilovic E. The value of radionuclide imaging in the diagnosis of sacroiliac joint syndrome. Spine (Phila Pa 1976) 1996; 21:2251-2254.

153. Slipman CW, Jackson HB, Lipetz JS, Chan KT, Lenrow D, Vresilovic EJ. Sacroiliac joint pain referral zones. Arch Phys Med Rehabil 2000; 81:334-338.

154. Laslett M, Aprill CN, McDonald B Young SB. Diagnosis of sacroiliac joint pain: A validity of individual provocation tests and composites of tests. Man Ther 2005; 10:207-218.

155. Young S, Aprill CN, Laslett M. Correlation of clinical examination characteristics with three sources of chronic low back pain. Spine J 2003; 3:460-465.

156. Maigne JY, Boulahdour H, Chatellier G. Value of quantitative radionuclide bone scanning in the diagnosis of sacroiliac joint syndrome in 32 patients with low back pain. Eur Spine J 1998; 7:328-331.

157. Broadhurst NA, Bond MJ. Pain provocation tests for the assessment of sacroiliac joint dysfunction. ] Spinal Disord 1998; 11:341-345.

158. Fukui S, Nosaka S. Pain patterns originating from the sacroiliac joints. J Anesth 2002; 16:245-247.

159. Laslett M, Young SB, Aprill CN, McDonald B. Diagnosing painful sacroiliac joints: A validity study of a McKenzie evaluation and sacroiliac provocation tests. Aust J Physiother 2003; 49:89-97.

16o. van der Wurff P, Buijs EJ, Groen G). A multitest regimen of pain provocation tests as an aid to reduce unnecessary minimally invasive sacroiliac joint procedures. Arch Phys Med Rehabil 2006; 87:10-14.

161. van der Wurff P, Buijs EJ, Groen GJ. Intensity mapping of pain referral areas in sacroiliac joint pain patients. J Manipulative Physiol Ther 2006; 29:190-195.

162. Fortin JD, Dwyer AP, West S, Pier J. Sacroiliac joint: Pain referral maps upon applying a new injection/arthrography technique. Part I: Asymptomatic volun- teers. Spine (Phila Pa 1976) 1994; 19:14751482

163. Fortin JD, Aprill CN, Ponthieux B, Pier J. Sacroiliac joint: Pain referral maps upon applying a new injection/arthrography technique. Part II: Clinical evaluation. Spine (Phila Pa 1976) 1994; 19:1483-1489.

164. Jung $\mathrm{JH}$, Kim HI, Shin DA, Shin DG, Lee JO, Kim HJ, Chung JH. Usefulness of pain distribution pattern assessment in decision-making for the patients with lumbar zygapophyseal and sacroiliac joint arthropathy. J Korean Med Sci 2007; 22:1048-1054.

165. Murakami E, Aizawa T, Noguchi K, Kanno $\mathrm{H}$, Okuno $\mathrm{H}$, Uozumi $\mathrm{H}$. Diagram specific to sacroiliac joint pain site indicated by one-finger test. J Orthop Sci 2008; 13:492-497.

166. Hartung W, Ross CJ, Straub R, Feuerbach S, Schölmerich J, Fleck M, Herold T. Ultrasound-guided sacroiliac joint injection in patients with established sacroiliitis: Precise IA injection verified by MRI scanning does not predict clinical outcome. Rheumatology (Oxford) 2010; 49:1479-1482.

167. Heuft-Dorenbosch L, Weijers R, Landewé $\mathrm{R}$, van der Linden $\mathrm{S}$, van der Heijde D. Magnetic resonance imaging changes of sacroiliac joints in patients with recent-onset inflammatory back pain: Inter-reader reliability and prevalence of abnormalities. Arthritis Res Ther 2006; 8:Rı1.

168. Madsen KB, Jurik AG. Magnetic resonance imaging grading system for active and chronic spondylarthritis changes in the sacroiliac joint. Arthritis Care Res (Hoboken) 2010; 62:11-18.

169. Klauser A, De Zordo T, Feuchtner G, Sögner P, Schirmer M, Gruber J, Sepp $\mathrm{N}$, Moriggl B. Feasibility of ultrasoundguided sacroiliac joint injection considering sonoanatomic landmarks at two different levels in cadavers and patients. Arthritis Rheum 2008; 59:1618-1624.

170. Harmon D, Alexiev V. Sonoanatomy and injection technique of the iliolumbar ligament. Pain Physician 2011; 14:469474.

171. Fritz J, Tzaribachev N, Thomas C, Carrino JA, Claussen CD, Lewin JS, Pereira $P L$. Evaluation of MR imaging guided steroid injection of the sacroiliac joints for the treatment of children with refractory enthesitis-related arthritis. Eur Radiol 2011; 21:1050-1057.

172. Gupta S. Double needle technique: An alternative method for performing diffi- 
cult sacroiliac joint injections. Pain Physician 2011; 14:281-284.

173. Hart R, Wendsche P, Koiš J, Komzák M, Okál F, Krejzla J. Injection of anaesthetic-corticosteroid to relieve sacroiliac joint pain after lumbar stabilization. Acta Chir Orthop Traumatol Cech 2011; 78:339-342.

174. Morimoto D, Isu T, Kim K, Matsumoto $\mathrm{R}$, Isobe M. Unexplained lower abdominal pain associated with sacroiliac joint dysfunction: Report of 2 cases. J Nihon Med Sch 2011; 78:257-260.

175. Stanford G, Burnham RS. Is it useful to repeat sacroiliac joint provocative tests post-block? Pain Med 2010; 11:1774-1776.

176. Klauser AS, De Zordo T, Feuchtner GM Djedovic G, Weiler RB, Faschingbauer $R$, Schirmer M, Moriggl B. Fusion of real-time US with CT images to guide sacroiliac joint injection in vitro and in vivo. Radiology 2010; 256:547-553.

177. Hamauchi S, Morimoto D, Isu T, Sugawara A, Kim K, Shimoda Y, Motegi H, Matsumoto $R$, Isobe $M$. Sacroiliac joint dysfunction presented with acute low back pain: Three case reports. No Shinkei Geka 2010; 38:655-661.

178. Migliore A, Bizzi E, Massafra U, Vacca F, Martin-Martin LS, Granata M, Tormenta S. A new technical contribution for ultrasound-guided injections of sacroiliac joints. Eur Rev Med Pharmacol Sci 2010; 14:465-469.

179. Streitparth F, Walter T, Wonneberger U, Chopra S, Wichlas F, Wagner M, Hermann KG, Hamm B, Teichgräber U. Image-guided spinal injection procedures in open high-field MRI with vertical field orientation: Feasibility and technical features. Eur Radiol 2010; 20:395-403.

180. Khurana A, Guha AR, Mohanty K, Ahu ja S. Percutaneous fusion of the sacroiliac joint with hollow modular anchorage screws: Clinical and radiological outcome. J Bone Joint Surg Br 2009; 91:627631.

181. Dreyfuss P, Snyder BD, Park K, Willard F, Carreiro J, Bogduk N. The ability of single site, single depth sacral lateral branch blocks to anesthetize the sacroiliac joint complex. Pain Med 2008; 9:844850.

182. Dreyfuss P, Henning T, Malladi N, Goldstein B, Bogduk N. The ability of multisite, multi-depth sacral lateral branch blocks to anesthetize the sacroiliac joint complex. Pain Med 2009; 10:679-688.

183. Sadreddini S, Noshad H, Molaeefard M, Ardalan MR, Ghojazadeh M, Shakouri
SK. Unguided sacroiliac injection: Effect on refractory buttock pain in patients with spondyloarthropathies. Presse Med 2009; 38:710-716.

184. Borowsky CD, Fagen G. Sources of sacroiliac region pain: Insights gained from a study comparing standard intra-articular injection with a technique combining intra- and peri-articular injection. Arch Phys Med Rehabil 2008; 89:20482056.

185. Harmon D, O'Sullivan M. Ultrasoundguided sacroiliac joint injection technique. Pain Physician 2008; 11:543-547.

186. Günaydin I, Pereira PL, Fritz J, König C, Kötter I. Magnetic resonance imaging guided corticosteroid injection of sacroiliac joints in patients with spondylarthropathy. Are multiple injections more beneficial? Rheumatol Int 2006; 26:396400

187. Murakami E, Tanaka Y, Aizawa T, Ishizuka M, Kokubun S. Effect of periarticular and intraarticular lidocaine injections for sacroiliac joint pain: Prospective comparative study. ] Orthop Sci 2007; 12:274-280.

188. Haufe SM, Mork AR. Sacroiliac joint debridement: A novel technique for the treatment of sacroiliac joint pain. Photomed Laser Surg 2005; 23:596-598.

189. Laslett M, McDonald B, Tropp H, Aprill $\mathrm{CN}$, Oberg B. Agreement between diagnoses reached by clinical examination and available reference standards: A prospective study of 216 patients with lumbopelvic pain. BMC Musculoskelet Disord 2005; 6:28.

190. Pekkafahli MZ, Kiralp MZ, Baekim CC, Silit E, Mutlu H, Oztürk E, Kizilkaya E, Dursun $H$. Sacroiliac joint injections performed with sonographic guidance. J Ultrasound Med 2003; 22:553-559.

191. Hansen HC. Is fluoroscopy necessary for sacroiliac joint injections? Pain Physician 2003; 6:155-158.

192. Rosenberg JM, Quint TJ, de Rosayro AM. Computerized tomographic localization of clinically-guided sacroiliac joint injections. Clin J Pain 2000; 16:18-21.

193. Pereira PL, Günaydin I, Duda SH, Trübenbach J, Rémy CT, Kötter I, Kastler $B$, Claussen CD. Corticosteroid injections of the sacroiliac joint during magnetic resonance: Preliminary results. J Radiol 2000; 81:223-226.

194. Liliang PC, Lu K, Liang CL, Tsai YD, Wang KW, Chen HJ. Sacroiliac joint pain after lumbar and lumbosacral fusion: Findings using dual sacroiliac joint blocks. Pain Med 2011; 12:565-570.

195. Laplante BL, Ketchum, JM, Saullo TR, DePalma MJ. Multivariable analysis of the relationship between pain referral patterns and the source of chronic low back pain. Pain Physician 2012; 15:171178.

196. Bokov A, Isrelov A, Skorodumov A, Aleynik A, Simonov A, Mlyavykh S. An analysis of reasons for failed back surgery syndrome and partial results after different types of surgical lumbar nerve root decompression. Pain Physician 2011; 14:545-557.

197. Weksler N, Velan GJ, Semionov M, Gurevitch B, Klein M, Rozentsveig V, Rudich T. The role of sacroiliac joint dysfunction in the genesis of low back pain: The obvious is not always right. Arch Orthop Trauma Surg 2007; 127:885-888.

198. Saha AK, Shah VM, Vakhariya V, Shah J, Horn JL. To do or not to do under fluoroscopy, that is the question: An analysis of sacroiliac joint and caudal epidural injections in a pain center. Am J Anesthesiol 1999; 26:269-271.

199. Morimoto D, Isu T, Shimoda Y, Hamauchi S, Sasamori T, Sugawara A, Kim K, Matsumoto R, Isobe $M$. Assessing the treatment for sacroiliac joint dysfunction, piriformis syndrome and tarsal tunnel syndrome associated with lumbar degenerative disease. No Shinkei Geka 2009; 37:873-879.

200. Simpson R, Gemmell H. Accuracy of spinal orthopaedic tests: A systemic review. Chiropr Osteopat 2006; 14:26.

201. Leboeuf $C$. The sensitivity and specificity of seven lumbo-pelvic orthopedic tests and the arm-fossa test. J Manip Physiol Ther 1990; 13:138-143.

202. Puhakka KB, Jurik AG, Schiottz-Christensen B, Hansen GV, Egund N, Christiansen JV, Stengaard-Pedersen K. MRI abnormalities of sacroiliac joints in early spondyloarthropathy: A 1-year follow-up study. Scand J Rheumatol 2004; 33:332338.

203. Puhakka KB, Melson F, Jurik AG, Boel LW, Vesterby A, Egund N. MR imaging of the normal sacroiliac joint with correlation to histology. Skeletal Radiol 2004; 33:15-28.

204. Vogler JB 3rd, Brown WH, Helms CA, Genant HK. The normal sacroiliac joint: A CT study of asymptomatic patients. Radiology 1984; 151:433-437.

205. Elgafy $H$, Semaan HB, Ebraheim NA, Coombs RJ. Computed tomography findings in patients with sacroiliac pain. 
Clin Orthop Relat Res 2001; 382:112-118.

206. Fortin JD, Tochin RB. Sacroiliac arthrograms and post-arthrography computerized tomography. Pain Physician 2003; 6:287-290.

207. Makki D, Khazim R, Zaidan AA, Ravi K, Toma T. Single photon emission computerized tomography (SPECT) scanpositive facet joints and other spinal structures in a hospital-wide population with spinal pain. Spine ] 2010; 10:58-62.

208. Algin O, Gokalp G, Baran B, Ocakoglu G, Yazici Z. Evaluation of sacroiliitis: Contrast-enhanced MRI with subtraction technique. Skeletal Radiol 2009; 38:983-988.

209. Fritz J, Henes JC, Thomas C, Clasen S, Fenchel M, Claussen CD, Lewin JS, Pereira PL. Diagnostic and interventional MRI of the sacroiliac joints using a 1.5-T open-bore magnet: A one-stopshopping approach. AJR Am J Roentgenol 2008; 191:1717-1724.

210. Yoshioka H, Nakano T, Kandatsu S, Koga M, Itai Y, Tsujii H. MR imaging of radiation osteitis in the sacroiliac joints. Magn Reson Imaging 2000; 18:125-128.

211. Lurie JD. What diagnostic tests are useful for low back pain? Best Pract Res Clin Rheumatol 2005; 19:557-575.

212. Lim R, Fahey FH, Drubach LA, Connolly LP, Treves ST. Early experience with fluorine-18 sodium fluoride bone PET in young patients with back pain. J Pediatr Orthop 2007; 27:277-282.

213. Feng $F, Y u W$, Yan $H$, Jiang M. Comparison of radiographs and magnetic resonance imaging in the detection of sacroiliitis in patients with ankylosing spondylitis. Zhongguo Yi Xue Ke Xue Yuan Xue Bao 1997; 19:185-191.

214. Wittram $\mathrm{C}$, Whitehouse $\mathrm{GH}$, Williams JW, Bucknall RC. A comparison of MR and $\mathrm{CT}$ in suspected sacroiliitis. J Comput Assist Tomogr 1996; 20:68-72.

215. Stürzenbecher $A$, Braun J, Paris $S$, Biedermann T, Hamm B, Bollow M. MR imaging of septic sacroiliitis. Skeletal Radiol 2000; 29:439-446.

216. Yu W, Feng F, Dion E, Yang H, Jiang M, Genant HK. Comparison of radiography, computed tomography and magnetic resonance imaging in the detection of sacroiliitis accompanying ankylosing spondylitis. Skeletal Radiol 1998; 27-311-320.

217. Puhakka KB, Jurik AG, Egund N, Schiottz-Christensen B, Stengaard-Pedersen K, van Overeem Hansen G, Christiansen JV. Imaging of sacroiliitis in early se- ronegative spondylarthropathy. Assessment of abnormalities by MR in comparison with radiography and CT. Acta Radiol 2003; 44:218-229.

218. Klein MA, Winalski CS, Wax MR, Piwnica-Worms DR. MR imaging of septic sacroiliitis. J Comput Assist Tomogr 1991; 15:126-132.

219. Docherty P, Mitchell MJ, MacMillan L, Mosher D, Barnes DC, Hanly JG. Magnetic resonance imaging in the detection of sacroiliitis. J Rheumatol 1992; 19:393-401.

220. Ahlström H, Feltelius N, Nyman R, Hällgren R. Magnetic resonance imaging of sacroiliac joint inflammation. Arthritis Rheum 1990; 33:1763-1769.

221. lovane A, Midiri M, Finazzo M, Mercurio G, Sallì L, Pappalardo A, Lagalla R. Magnetic resonance imaging of seronegative sacroiliitis. Radiol Med 1998; 96:185189.

222. Bollow M, Braun J, Biedermann T, Mutze S, Paris S, Schauer-Petrowskaja $C$, Minden K, Schmitz SA, Schöntube M, Hamm B. Use of contrast-enhanced MR imaging to detect sacroiliitis in children. Skeletal Radiol 1998; 27:606-616.

223. Fewins HE, Whitehouse GH, Bucknall RC. Role of computed tomography in the evaluation of suspected sacroiliac joint disease. J R Soc Med 1990; 83:430432.

224. Geijer M, Göthlin GG, Göthlin JH. Observer variation in computed tomography of the sacroiliac joints: A retrospective analysis of 1383 cases. Acta Radiol 2007; 48:665-671.

225. Guglielmi G, De Serio A, Leone A, Cammisa M. Imaging of sacroiliac joints. Rays 2000; 25:63-74.

226. Hermann KG, Braun J, Fischer T, Reisshauer $\mathrm{H}$, Bollow M. Magnetic resonance tomography of sacroiliitis: Anatomy, histological pathology, MR-morphology, and grading. Radiologe 2004; 44:217-228.

227. Geijer M, Gadeholt Göthlin G, Göthlin $\mathrm{JH}$. The validity of the New York radiological grading criteria in diagnosing sacroiliitis by computed tomography. Acta Radiol 2009; 50:664-673.

228. Hanly JG, Mitchell MJ, Barnes DC, MacMillan L. Early recognition of sacroiliitis by magnetic resonance imaging and single photon emission computed tomography. J Rheumatol 1994; 21:20882095.

229. Blum U, Buitrago-Tellez C, Mundinger
A, Krause T, Laubenberger J, Vaith P, Peter $\mathrm{HH}$, Langer $\mathrm{M}$. Magnetic resonance imaging (MRI) for detection of active sacroiliitis--a prospective study comparing conventional radiography, scintigraphy, and contrast enhanced MRI. J Rheumatol 1996; 23:2107-2115.

230. Braun J, Sieper J, Bollow M. Imaging of sacroiliitis. Clin Rheumatol 2000; 19:5157.

231. Miron SD, Khan MA, Wiesen EJ, Kushner I, Bellon EM. The value of quantitative sacroiliac scintigraphy in detection of sacroiliitis. Clin Rheumatol 1983; 2:407-414.

232. Borlaza GS, Seigel R, Kuhns LR, Good AE, Rapp R, Martel W. Computed tomography in the evaluation of sacroiliac arthritis. Radiology 1981; 139:437-440.

233. Williamson L, Dockerty JL, Dalbeth N, McNally E, Ostlere S, Wordsworth BP. Clinical assessment of sacroiliitis and HLA-B27 are poor predictors of sacroiliitis diagnosed by magnetic resonance imaging in psoriatic arthritis. Rheumatology (Oxford) 2004; 43:85-88.

234. Guglielmi G, Cascavilla A, Scalzo G, Carotti M, Salaffi F, Grassi W. Imaging findings of sacroiliac joints in spondyloarthropathies and other rheumatic conditions. Radiol Med 2011; 116:292-301.

235. Lawson TL, Foley WD, Carrera GF, Berland LL. The sacroiliac joints: Anatomic, plain roentgenographic, and computed tomographic analysis. J Comput Assist Tomogr 1982; 6:307-314.

236. Le Blanche AF, Mabi C, Bigot JM, Rousseau J, Trèves R, Outrequin G, Dupuy JP, Caix M. The sacroiliac joint: Anatomical study in the coronal plane and MR correlation. Surg Radiol Anat 1996; 18:215220.

237. Brandt J, Bollow M, Häberle J, Rudwaleit M, Eggens U, Distler A, Sieper J, Braun J. Studying patients with inflammatory back pain and arthritis of the lower limbs clinically and by magnetic resonance imaging: Many, but not all patients with sacroiliitis have spondyloarthropathy. Rheumatology (Oxford) 1999; 38:831-836.

238. Gupta AD. Sacroiliac joint pathologies in low back pain. J Back Musculoskelet Rehabil 2009; 22:91-97.

239. Tuite MJ. Sacroiliac joint imaging. Semin Musculoskelet Radiol 2008; 12:72-82.

240. Jurik AG. Technique and radiation dose of conventional $\mathrm{X}$-rays and computed tomography of the sacroiliac joint [in German]. Radiologe 2004; 44:229-233. 
241. Manchikanti L, Singh V, Falco FJE, Cash KA, Pampati V. Evaluation of lumbar facet joint nerve blocks in managing chronic low back pain: A randomized, doubleblind, controlled trial with a 2-year follow-up. Int J Med Sci 2010; 7:124-135.

242. Manchikanti L, Singh V, Falco FJE, Cash KA, Fellows B. Comparative outcomes of a 2-year follow-up of cervical medial branch blocks in management of chronic neck pain: A randomized, double-blind controlled trial. Pain Physician 2010; 13:437-450.

243. Manchikanti L, Singh V, Falco FJE, Cash KA, Pampati V, Fellows B. Comparative effectiveness of a one-year follow-up of thoracic medial branch blocks in management of chronic thoracic pain: A randomized, double-blind active controlled trial. Pain Physician 2010; 13:535-548.

244. Lord SM, Barnsley L, Wallis BJ, McDonald GJ, Bogduk N. Percutaneous radiofrequency neurotomy for chronic cervical zygapophyseal-joint pain. $N$ Engl J Med 1996; 335:1721-1726.

245. Nath S, Nath CA, Pettersson K. Percutaneous lumbar zygapophysial (facet) joint neurotomy using radiofrequency current, in the management of chronic low back pain. A randomized double-blind trial. Spine (Phila Pa 1976) 2008; 33:12911297.

246. Smuck M, Levin JH. RE: Manchikanti L, Singh V, Falco FJE, Cash KA, Fellows B. Cervical medial branch blocks for chronic cervical facet joint pain: A randomized double-blind, controlled trial with one-year follow-up. Spine (Phila Pa 1976) 2008; 33:1813-1820. Spine (Phila Pa 1976) 2009; 34:1116-1117.

247. Manchikanti L, Benyamin RM, Falco FJE, Caraway DL, Datta S, Hirsch JA. Guidelines warfare over interventional techniques: Is there a lack of discourse or straw man? Pain Physician 2012; 15: E1E26.

248. Chou R, Atlas SJ, Loeser JD, Rosenquist RW, Stanos SP. Guideline warfare over interventional therapies for low back pain: Can we raise the level of discourse? J Pain 2011; 12:833-839.

249. Shah RV, Everett CR, McKenzie-Brown AM, Sehgal N. Discography as a diagnostic test for spinal pain: A systematic and narrative review. Pain Physician 2005; 8:187-209.

250. Carragee EJ, Tanner CM, Yang B, Brito JL, Truong T. False-positive findings on lumbar discography. Reliability of subjective concordance assessment during provocative disc injection. Spine (Phila Pa 1976) 1999; 24:2542-2547.

251. Laslett M, Oberg B, Aprill CN, McDonald B. Centralization as a predictor of provocation discography results in chronic low back pain, and the influence of disability and distress on diagnostic power. Spine J 2005; 5:370-380.

252. Laslett M, McDonald B, Aprill CN, Tropp H, Oberg B. Clinical predictors of screening lumbar zygapophyseal joint blocks: Development of clinical prediction rules. Spine ] 2006; 6:370-379.

253. Laslett M, Aprill CN, McDonald B, Oberg B. Clinical predictors of lumbar provocation discography: A study of clinical predictors of lumbar provocation discography. Eur Spine J 2006; 15:1473-1484.

254. North RB, Kidd DH, Zahurak M, Piantadosi S. Specificity of diagnostic nerve blocks: A prospective, randomized study of sciatica due to lumbosacral spine disease. Pain 1996; 65:77-85.

255. Bogduk N. In: Defense of King et al: The validity of manual examination in assessing patients with neck pain. Spine ] 2007; 7:749-752; author reply (Carragee EJ) $752-753$.

256. Carragee EJ, Hurwitz EL, Cheng I, Carroll LJ, Nordin M, Guzman J, Peloso P, Holm LW, Côté P, Hogg-Johnson S, van der Velde G, Cassidy JD, Haldeman S; Bone and Joint Decade 2000-2010 Task Force on Neck Pain and Its Associated Disorders. Treatment of neck pain: Injections and surgical interventions: Results of the Bone and Joint Decade 2000-2010 Task Force on Neck Pain and Its Associated Disorders. Spine (Phila Pa 1976) 2008; 33:S153-S169.

257. Hogan QH, Abram SE. Neural blockade for diagnosis and prognosis. A review. Anesthesiology 1997; 86:216-241.

258. Hildebrandt J. Relevance of nerve blocks in treating and diagnosing low back pain — Is the quality decisive? Schmerz 2001; 15:474-483.

259. Saal JS. General principles of diagnostic testing as related to painful lumbar spine disorders: A critical appraisal of current diagnostic techniques. Spine (Phila Pa 1976) 2002; 27:2538-2545.

26o. Cohen SP, Raja SN. Pathogenesis, diagnosis, and treatment of lumbar zygapophysial (facet) joint pain. Anesthesiology 2007; 106:591-614.

261. Ackerman WE, Munir MA, Zhang JM, Ghaleb A. Are diagnostic lumbar facet injections influenced by pain of muscu- lar origin? Pain Pract 2004; 4:286-291.

262. Cohen SP, Williams KA, Kurihara C, Nguyen C, Shields C, Kim P, Griffith SR, Larkin TM, Crooks M, Williams N, Morlando B, Strassels SA. Multicenter, randomized, comparative cost-effectiveness study comparing 0,1 , and 2 diagnostic medial branch (facet joint nerve) block treatment paradigms before lumbar facet radiofrequency denervation. Anesthesiology 2010; 113:395-405.

263. Manchikanti L, Boswell MV, Manchukonda R, Cash KA, Giordano J. Influence of prior opioid exposure on diagnostic facet joint nerve blocks. J Opioid Manage 2008; 4:351-360.

264. Manchikanti L, Pampati V, Damron KS, McManus CD, Jackson SD, Barnhill RC, Martin JC. The effect of sedation on diagnostic validity of facet joint nerve blocks: An evaluation to assess similarities in population with involvement in cervical and lumbar regions (ISRCTNo: 76376497). Pain Physician 2006; 9:47-52.

265. Manchikanti L, Damron KS, Rivera J, McManus CD, Jackson SD, Barnhill RC, Martin JC. Evaluation of effect of sedation as a confounding factor in the diagnostic validity of lumbar facet joint pain: A prospective, randomized, double-blind, placebo-controlled evaluation. Pain Physician 2004; 7:411-417.

266. Manchikanti L, Pampati V, Damron KS, McManus CD, Jackson SD, Barnhill RC, Martin JC. A randomized, prospective, double-blind, placebo-controlled evaluation of the effect of sedation on diagnostic validity of cervical facet joint pain. Pain Physician 2004; 7:301-309.

267. Manchikanti L, Pampati V, Damron KS. The role of placebo and nocebo effects of perioperative administration of sedatives and opioids in interventional pain management. Pain Physician 2005; 8:349-355.

268. Manchikanti L, Giordano J, Fellows B, Hirsch JA. Placebo and nocebo in interventional pain management: $A$ friend or a foe - or simply foes? Pain Physician 2011; 14:E157-E175.

269. Cohen SP, Stojanovic MP, Crooks M, Kim P, Shields CH, Schmidt RK, Hurley RW. Lumbar zygapophysial (facet) joint radiofrequency denervation success as a function of pain relief during diagnostic medial branch blocks: A multi-center analysis. Spine J 2008; 8: 498-504.

270. Weller SC, Mann NC. Assessing rater performance without a "gold standard" using consensus theory. Med Decis Mak- 
ing 1997; 17:71-79.

271. Pearl WS. A hierarchal outcome approach to test assessment. Ann Emerg Med 1999; 33:77-84.

272. Laslett $M$, Williams $M$. The reliability of selected pain provocation tests for sacroiliac joint pathology. Spine (Phila Pa 1976) 1994; 19:1243-1249.

273. Laslett M. Pain provocation sacroiliac joint tests: Reliability and prevalence. In: Vleeming A, Monney V, Snijders C), Dormann TA, Steickart R (eds). Movement, Stability and Low Back Pain: The Essential Role of the Pelvis. 1st edition. Churchill Livingstone, New York, 1997.

274. Kokmeyer DJ, Van der Wurff P, Aufdemkampe G, Fickenscher TC. The reliability of multitest regimens with sacroiliac pain provocation tests. J Manipulative Physiol Ther 2002; 25:42-48.

275. van der Wurff P, Hagmeijer RH, Meyne W. Clinical tests of the sacroiliac joint. A systematic methodological review. Part 1: Reliability. Man Ther 2000; 5:30-36.

276. Robinson HS, Brox JI, Robinson R, Bjelland E, Solem S, Telje T. The reliability of selected motion- and pain provocation tests for the sacroiliac joint. Man Ther 2007;12:72-79.

277. Schwarzer AC, Aprill CN, Derby R, Fortin J, Kine G, Bogduk N. The relative contributions of the disc and zygapophyseal joint in chronic low back pain. Spine (Phila Pa 1976) 1994; 19:801-806.

278. McKenzie RA. The Lumbar Spine: Mechanical Diagnosis and Therapy. Spinal Publications Ltd, Wiakanae, NZ, 1981.

279. Kilpikoski S, Airaksinen O, Kankaanpää $M$, Leminen P, Videman T, Alen M. Interexaminer reliability of low back pain assessment using the McKenzie method. Spine (Phila Pa 1976) 2002; 27:E207E214.

280. Donelson R, Silva G, Murphy K. Centralization phenomenon. Its usefulness in evaluating and treating referred pain. Spine (Phila Pa 1976) 1990; 15:211-213.

281. Donelson R, Aprill C, Medcalf R, Grant W. A prospective study of centraliza- tion of lumbar and referred pain. A predictor of symptomatic discs and anular competence. Spine (Phila Pa 1976) 1997; 22:1115-1122.

282. Long A, Donelson R, Fung T. Does it matter which exercise? A randomized control trial of exercise for low back pain. Spine (Phila Pa 1976) 2004; 29:25932602.

283. Werneke MW, Hart DL. Centralization: Association between repeated endrange pain responses and behavioral signs in patients with acute non-specific low back pain. J Rehabil Med 2005; 37:286-290.

284. Werneke M, Hart DL. Discriminant validity and relative precision for classifying patients with nonspecific neck and back pain by anatomic pain patterns. Spine (Phila Pa 1976) 2003; 28:161-166.

285. Manchikanti L, Falco FJ, Benyamin RM, Helm S 2nd, Parr AT, Hirsch JA. The impact of comparative effectiveness research on interventional pain management: Evolution from Medicare Modernization Act to Patient Protection and Affordable Care Act and the PatientCentered Outcomes Research Institute. Pain Physician 2011; 14:E249-E282.

286. Carette S, Marcoux S, Truchon R, Grondin C, Gagnon J, Allard Y, Latulippe M. A controlled trial of corticosteroid injections into facet joints for chronic low back pain. N Engl J Med 1991; 325:10021007.

287. Carette S, Leclaire R, Marcoux S, Morin F, Blaise GA, St-Pierre A, Truchon $R$, Parent $F$, Levesque J, Bergeron $V$, Montminy $\mathrm{P}$, Blanchette $\mathrm{C}$. Epidural corticosteroid injections for sciatica due to herniated nucleus pulposus. N Engl J Med 1997; 336:1634-1640.

288. Gupta AK, Mital VK, Azmi RU. Observations of the management of lumbosciatic syndromes (sciatica) by epidural saline.J Indian Med Assoc 1970; 54:194-196.

289. Indahl A, Kaigle AM, Reikeräs $\mathrm{O}, \mathrm{Holm}$ $\mathrm{SH}$. Interaction between the porcine lumbar intervertebral disc, zygapophysi- al joints, and paraspinal muscles. Spine (Phila Pa 1976) 1997; 22:2834-2840.

29o. Indahl A, Kaigle A, Reikerås $\mathrm{O}$, Holm S. Electromyographic response of the porcine multifidus musculature after nerve stimulation. Spine (Phila Pa 1976) 1995; 20:2652-2658.

291. Pham Dang C, Lelong A, Guilley J, Nguyen JM, Volteau C, Venet G, Perrier C, Lejus C, Blanloeil Y. Effect on neurostimulation of injectates used for perineural space expansion before placement of a stimulating catheter: Normal saline versus dextrose $5 \%$ in water. Reg Anesth Pain Med 2009; 34:398-403.

292. Tsui BC, Kropelin B, Ganapathy S, Finucane B. Dextrose $5 \%$ in water: Fluid medium maintaining electrical stimulation of peripheral nerve during stimulating catheter placement. Acta Anaesthesiol Scand 2005; 49:1562-1565.

293. Pasqualucci A, Varrassi G, Braschi A, Peduto VA, Brunelli A, Marinangeli F, Gori F, Colò F, Paladín A, Mojoli F. Epidural local anesthetic plus corticosteroid for the treatment of cervical brachial radicular pain: Single injection verus continuous infusion. Clin J Pain 2007; 23:551-557.

294. Pasqualucci A. Experimental and clinical studies about the preemptive analgesia with local anesthetics. Possible reasons of the failure. Minerva Anestesiol 1998; 64:445-457.

295. Sato C, Sakai A, Ikeda Y, Suzuki H, Sakamoto $A$. The prolonged analgesic effect of epidural ropivacaine in a rat model of neuropathic pain. Anesth Analg 2008; 106:313-320.

296. Tachihara H, Sekiguchi M, Kikuchi S, Konno S. Do corticosteroids produce additional benefit in nerve root infiltration for lumbar disc herniation. Spine (Phila Pa 1976) 2008; 33:743-747.

297. Ghahreman A, Bogduk N. Predictors of a favorable response to transforaminal injection of steroids in patients with lumbar radicular pain due to disc herniation. Pain Med 2011; 12:871-879. 